\title{
Interacting Fermi liquid in three dimensions at finite temperature: Part I: Convergent Contributions
}

\author{
M. Disertori ${ }^{a}$, J. Magnen $^{b}$ and V. Rivasseau ${ }^{b}$ \\ November 10, 2018 \\ a) Institute for Advanced Study \\ Einstein Drive, Princeton \\ NJ 08540, USA \\ b) Centre de Physique Théorique, CNRS UMR 7644 \\ Ecole Polytechnique \\ 91128 Palaiseau Cedex, FRANCE
}

\begin{abstract}
In this paper we complete the first step, namely the uniform bound on completely convergent contributions, towards proving that a three dimensional interacting system of Fermions is a Fermi liquid in the sense of Salmhofer. The analysis relies on a direct space decomposition of the propagator, on a bosonic multiscale cluster expansion and on the Hadamard inequality, rather than on a Fermionic expansion and an angular analysis in momentum space, as was used in the recent proof by two of us of Salmhofer's criterion in two dimensions.
\end{abstract}

\section{Introduction}

Conducting electrons in a metal at low temperature are well described by Fermi liquid theory. However we know that the Fermi liquid theory is not valid down to zero temperature. Indeed below the BCS critical temperature 
the dressed electrons or holes which are the excitations of the Fermi liquid bound into Cooper pairs and the metal becomes superconducting. Even when the dominant electron interaction is repulsive, the Kohn-Luttinger instabilities prevent the Fermi liquid theory to be generically valid down to zero temperature.

Hence Fermi liquid theory (e.g. for the simplest case of a jellium model with a spherical Fermi surface) is only an effective theory above some nonperturbative transition temperature, and it is not obvious to precise its mathematical definition. Recently Salmhofer proposed such a mathematical definition [S]. It consists in proving that (under a suitable renormalization condition on the two-point function), perturbation theory is analytic in a domain $|\lambda \log T| \leq K$, where $\lambda$ is the coupling constant and $T$ is the temperature, and that uniform bounds hold in that domain for the self-energy and its first and second derivatives. This criterion in particular excludes Luttinger liquid behavior, which has been proved to hold in one dimension [BGPS$\mathrm{BM}]$, and for which second momentum-space derivatives of the self-energy are unbounded in that domain.

Recently two of us proved Salmhofer's criterion for the two dimensional jellium model [DR1-2]. However the proof relies in a key way on the special momentum conservation rules in two dimensions. In three dimensions general vertices are not necessarily planar in momentum space. This has drastic constructive consequences (although perturbative power counting is similar in 2 and 3 dimensions). In particular it seems to prevent, up to now, any constructive analysis based on angular decomposition in momentum space. The only existing constructive result for three dimensional Fermions relies on the use of a bosonic method (cluster expansion) together with the Hadamard inequality $[\mathrm{MR}]$. It proves that the radius of convergence of the theory in a single momentum slice of the renormalization group analysis around the Fermi surface is at least a constant independent of the slice.

In this paper, we build upon the analysis of [MR], extending it to many slices. We use a multiscale bosonic cluster expansion based on a direct space decomposition of the propagator, which is not the usual momentum decomposition around the Fermi sphere. We bound uniformly the sum of all convergent polymers in the Salmhofer domain $|\lambda \log T| \leq K$. Hence our result is the three dimensional analog of [FMRT] and [DR1]. Because of its technical nature, this result is stated precisely only in section III.6, after the definition of the multiscale cluster expansion.

Using a Mayer expansion we plan in a future paper (which would be the 
three dimensional analog of [DR2]) to perform renormalization of the two point subgraphs and to study boundedness of the self energy and of its first and second momentum space derivatives. That would complete the proof of Fermi liquid behavior in three dimensions.

Remark however that the optimal analyticity radius of the Fermi liquid series should be given by $|\lambda \ln T|=K_{B C S}$ where $K_{B C S}$ is a numerical constant given by the coefficient of a so called "wrong-way" bubble graph [FT2]. In this paper we prove analyticity in a domain $\lambda|\ln T| \leq K$ but our constant is not the expected optimal one, $K_{B C S}$, not only because of some lazy bounds, but also because of a fundamental difficulty linked to the use of the Hadamard inequality. Actually the kind of Hadamard bound relevant for a model of fermions with two spin states is $\sum_{n} \frac{\lambda^{n}}{n !} \operatorname{det}^{2} A_{n} \leq \frac{n^{n}}{n !} a^{n}$, where $A_{n}$ is an $n \times$ $n$ matrix whose coefficients are all bounded by $a$. Hence (using Stirling's formula), the radius of convergence in $\lambda$ of that series is only shown to be at least 1 /ea by this bound, whether $1 / a$ would be expected from perturbation theory.

For this reason it seems to us that the analyticity radius obtained by any method based on Hadamard bound is smaller than the optimal radius by a factor at least $1 / e$, and we do not know how to cure this defect.

\section{Model}

We consider the simple model of isotropic jellium in three spatial dimensions with a local four point interaction. We use the formalism of non-relativistic field theory at imaginary time of [FT1-2-BG] to describe the interacting fermions at finite temperature. Our model is therefore similar to the GrossNeveu model, but with a different, non relativistic propagator.

\section{II.1 Free propagator}

Using the Matsubara formalism, the propagator at temperature $T, C\left(x_{0}, \vec{x}\right)$, is antiperiodic in the variable $x_{0}$ with antiperiod $\frac{1}{T}$. This means that the Fourier transform defined by

$$
\hat{C}(k)=\frac{1}{2} \int_{-\frac{1}{T}}^{\frac{1}{T}} d x_{0} \int d^{3} x e^{-i k x} C(x)
$$


is not zero only for discrete values (called the Matsubara frequencies) :

$$
k_{0}=\frac{2 n+1}{\beta} \pi, \quad n \in \mathbb{Z},
$$

where $\beta=1 / T$ (we take $\not h=k=1$ ). Remark that only odd frequencies appear, because of antiperiodicity.

Our convention is that a four dimensional vector is denoted by $x=\left(x_{0}, \vec{x}\right)$ where $\vec{x}$ is the three dimensional spatial component. The scalar product is defined as $k x:=-k_{0} x_{0}+\vec{k} \cdot \vec{x}$. By some slight abuse of notations we may write either $C(x-\bar{x})$ or $C(x, \bar{x})$, where the first point corresponds to the field and the second one to the antifield (using translation invariance of the corresponding kernel).

Actually $\hat{C}(k)$ is obtained from the real time propagator by changing $k_{0}$ in $i k_{0}$ and is equal to:

$$
\hat{C}_{a b}(k)=\delta_{a b} \frac{1}{i k_{0}-e(\vec{k})}, \quad e(\vec{k})=\frac{\vec{k}^{2}}{2 m}-\mu,
$$

where $a, b \in\{\uparrow, \downarrow\}$ are the spin indices. The vector $\vec{k}$ is three-dimensional. Since our theory has three spatial dimensions and one time dimension, there are really four dimensions. The parameters $m$ and $\mu$ correspond to the effective mass and to the chemical potential (which fixes the Fermi energy). To simplify notation we put $2 m=\mu=1$, so that, if $\rho=|\vec{k}|, e(\vec{k})=e(\rho)=$ $\rho^{2}-1$. Hence,

$$
C_{a b}(x)=\frac{1}{(2 \pi)^{3} \beta} \sum_{k_{0}} \int d^{3} k e^{i k x} \hat{C}_{a b}(k) .
$$

The notation $\sum_{k_{0}}$ means really the discrete sum over the integer $n$ in (III.2). When $T \rightarrow 0$ (which means $\beta \rightarrow \infty$ ) $k_{0}$ becomes a continuous variable, the corresponding discrete sum becomes an integral, and the corresponding propagator $C_{0}(x)$ becomes singular on the Fermi surface defined by $k_{0}=0$ and $|\vec{k}|=1$. In the following to simplify notations we will write:

$$
\int d^{4} k \equiv \frac{1}{\beta} \sum_{k_{0}} \int d^{3} k \quad, \quad \int d^{4} x \equiv \frac{1}{2} \int_{-\beta}^{\beta} d x_{0} \int d^{3} x .
$$




\section{II.2 Ultraviolet cutoff}

It is convenient to add a continuous ultraviolet cut-off (at a fixed scale $\Lambda_{u}$ ) to the propagator (II.3) for two reasons: first because it makes its Fourier transformed kernel in position space well defined, and second because a non relativistic theory does not make sense anyway at high energies. To preserve physical (or Osterwalder-Schrader) positivity one should introduce this ultraviolet cutoff only on spatial frequencies [FT2]. However for convenience we introduce this cutoff both on spatial and on Matsubara frequencies as in [FMRT]; indeed the Matsubara cutoff could be lifted with little additional work. The propagator (II.3) equipped with this cut-off is called $C^{u}$ and is defined as:

$$
\hat{C}^{u}(k):=\left.\hat{C}(k)[u(r)]\right|_{r=k_{0}^{2}+e^{2}(\vec{k})}
$$

where the compact support function $0 \leq u(r) \in \mathcal{C}_{0}^{\infty}(\mathrm{R})$ satisfies: $u(r)=1$ for $r \leq 1, u(r)=0$ for $r>10$.

\section{II.3 Position space}

In the following we will use the propagator in position space. The key point for further analysis is to write it as

$$
C^{u}(\vec{x}, t)=\frac{1}{1+|\vec{x}|} \frac{1}{1+f(t)+|\vec{x}|} F(\vec{x}, t)
$$

where $f(t)$ is defined by

$$
f(t):=\left|\frac{\sin (2 \pi T t)}{2 \pi T}\right|=\varepsilon(t) \frac{\sin (2 \pi T t)}{2 \pi T} \quad t \in\left[-\frac{1}{T}, \frac{1}{T}\right]
$$

and $\varepsilon(t)$ is the sign of $\sin (2 \pi T t)$.

This is useful since the remaining function $F$ has a spatial decay scaled with $T$, and no global scaling factor in $T$, as proved in the following lemma.

Lemma 1 For any $p \geq 1$, there exists $K_{p}$ such that the function $F(\vec{x}, t)$ defined by (II.7) satisfies

$$
|F(\vec{x}, t)| \leq \frac{K_{p}}{(1+T|\vec{x}|)^{p}} \quad \forall p \geq 1
$$


Proof. In radial coordinates the propagator is written as

$$
C^{u}(\vec{x}, t)=\frac{T}{(2 \pi)^{3}} \sum_{k_{0}} \int_{0}^{2 \pi} d \phi \int_{0}^{\pi} d \theta \sin \theta \int_{0}^{\infty} d \rho \rho^{2} \frac{e^{i \rho|\vec{x}| \cos \theta-i k_{0} t}}{i k_{0}-e(\rho)} u\left[k_{0}^{2}+e^{2}(\rho)\right] .
$$

Now we write the integral over $\theta$ as

$$
\int_{0}^{\pi} d \theta \sin \theta e^{i \rho|\vec{x}| \cos \theta}=\int_{-1}^{1} d v e^{i \rho|\vec{x}| v}
$$

and applying twice the identity

$$
e^{i \rho|\vec{x}| v}=\frac{1}{(1+|\vec{x}|)}\left(1-\frac{i}{\rho} \frac{d}{d v}\right) e^{i \rho|\vec{x}| v}
$$

we obtain

$$
\begin{aligned}
& \int_{-1}^{1} d v e^{i \rho|\vec{x}| v}=\frac{1}{1+|\vec{x}|} \int_{-1}^{1} d v\left[1-\frac{i}{\rho} \frac{d}{d v}\right] e^{i \rho|\vec{x}| v} \\
& \quad=\frac{1}{1+|\vec{x}|}\left[\int_{-1}^{1} d v e^{i \rho|\vec{x}| v}+\frac{1}{i \rho}\left(e^{i \rho|\vec{x}|}-e^{-i \rho|\vec{x}|}\right)\right] \\
& \quad=\left[\frac{1}{(1+|\vec{x}|)^{2}} \int_{-1}^{1} d v e^{i \rho|\vec{x}| v}\right]+\left\{\left[\frac{(2+|\vec{x}|)}{(1+|\vec{x}|)^{2}}\right] \frac{1}{i \rho}\left(e^{i \rho|\vec{x}|}-e^{-i \rho|\vec{x}|}\right)\right\}
\end{aligned}
$$

We decompose further, introducing for the first term $1=\chi(|\vec{x}| \leq 1)+\chi(|\vec{x}|>$ 1 ), where $\chi$ is the characteristic function of the event indicated, and perform the $v$ integration for the second term only. In this way the function $F$ can be written as a sum of two terms $F=F_{1}+F_{2}$ where

$$
\begin{gathered}
F_{1}=\chi(|\vec{x}| \leq 1) \frac{(1+f(t)+|\vec{x}|)}{(1+|\vec{x}|)} \frac{T}{(2 \pi)^{2}} \\
\sum_{k_{0}} \int_{-1}^{1} d v \int_{0}^{\infty} d \rho \rho^{2} e^{i \rho|\vec{x}| v-i k_{0} t} \frac{u\left[k_{0}^{2}+e^{2}(\rho)\right]}{i k_{0}-e(\rho)} \\
F_{2}=\left[\frac{(2+|\vec{x}|+\chi(|\vec{x}|>1) /|\vec{x}|)(1+f(t)+|\vec{x}|)}{(1+|\vec{x}|)}\right] \frac{T}{(2 \pi)^{2}} \\
\sum_{k_{0}} \sum_{\sigma= \pm 1} \int_{0}^{\infty} d \rho \frac{\sigma \rho}{i} e^{i \sigma \rho|\vec{x}|-i k_{0} t} \frac{u\left[k_{0}^{2}+e^{2}(\rho)\right]}{i k_{0}-e(\rho)}
\end{gathered}
$$


Now we apply on $F_{1}$ and on $F_{2}$ the identity

$$
\left[1+f(t)-\frac{i}{2} \varepsilon(t) a_{i}|\vec{x}|\right] e^{i a_{i} \rho|\vec{x}|-i k_{0} t}=\left[1+\varepsilon(t)\left(i \frac{\Delta}{\Delta k_{0}}-\frac{1}{2} \frac{d}{d \rho}\right)\right] e^{i a_{i} \rho|\vec{x}|-i k_{0} t}
$$

where we defined $a_{1}=: v$ for $F_{1}$ and $a_{2}=: \sigma$ for $F_{2}$, and where the discretized derivative $\frac{\Delta}{\Delta k_{0}}$ on a function $F\left(k_{0}\right)$ is defined by

$$
\frac{\Delta}{\Delta k_{0}} F\left(k_{0}\right)=\frac{1}{4 \pi T}\left[F\left(k_{0}+2 \pi T\right)-F\left(k_{0}-2 \pi T\right)\right] .
$$

Hence integrating by parts the $F_{i}$ 's are written as

$$
\begin{gathered}
F_{1}(\vec{x}, t)=\frac{T}{(2 \pi)^{2}} \sum_{k_{0}} \int_{-1}^{1} d v f_{1}(\vec{x}, t, v) \int_{0}^{\infty} d \rho e^{i \rho|\vec{x}| v-i k_{0} t} G_{1}\left(k_{0}, \rho\right) \\
G_{1}\left(k_{0}, \rho\right)=[1+\varepsilon(t) \Delta]\left[\frac{\rho^{2} u\left(k_{0}^{2}+e^{2}(\rho)\right)}{i k_{0}-e(\rho)}\right] \\
F_{2}(\vec{x}, t)=\frac{T}{(2 \pi)^{2}} \sum_{k_{0}} \sum_{\sigma} f_{2}(\vec{x}, t, \sigma) \int_{0}^{\infty} d \rho \frac{\sigma}{i} G_{2}\left(k_{0}, \rho\right) e^{i \sigma \rho|\vec{x}|-i k_{0} t} \\
G_{2}\left(k_{0}, \rho\right)=[1+\varepsilon(t) \Delta]\left[\frac{\rho u\left(k_{0}^{2}+e^{2}(\rho)\right)}{i k_{0}-e(\rho)}\right]
\end{gathered}
$$

where we have defined

$$
\begin{gathered}
f_{1}(\vec{x}, t, v)=\chi(|\vec{x}| \leq 1) \frac{(1+f(t)+|\vec{x}|)}{(1+|\vec{x}|)\left(1+f(t)-\frac{i}{2} \varepsilon(t) v|\vec{x}|\right)} \\
f_{2}(\vec{x}, t, \sigma)=\frac{2+|\vec{x}|+\chi(|\vec{x}|>1) /|\vec{x}|}{1+|\vec{x}|} \frac{1+f(t)+|\vec{x}|}{\left(1+f(t)-\frac{i}{2} \varepsilon(t)|\vec{x}| \sigma\right)} \\
\Delta=\left(\frac{1}{2} \frac{d}{d \rho}-i \frac{\Delta}{\Delta k_{0}}\right)
\end{gathered}
$$

We remark that these functions are uniformly bounded in modulus $\left(f_{1}\right.$ is bounded by 1 and $f_{2}$ by 6 ). The signs and coefficients in $\Delta$ have been optimized in order to obtain a positive factor $1+f(t)$ and to minimize the 
action of $\Delta$ on $\left(i k_{0}-e(\rho)\right)^{-1}$. After a tedious but trivial computation, we find

$$
\begin{aligned}
& G_{i}=:[1+\epsilon(t) \Delta]\left[\rho^{b_{i}} \frac{u\left[k_{0}^{2}+e^{2}(\rho)\right]}{i k_{0}-e(\rho)}\right]=\left\{\rho^{b_{i}}+\frac{\epsilon(t) b_{i} \rho^{b_{i}-1}}{2}\right\} \frac{u\left[k_{0}^{2}+e^{2}(\rho)\right]}{i k_{0}-e(\rho)} \\
& +\rho^{b_{i}} \epsilon(t)\left\{u^{\prime}\left[k_{0}^{2}+e^{2}(\rho)\right]\left[\frac{2 \rho\left(\rho^{2}-1\right)}{i k_{0}-e(\rho)}-\frac{i k_{0}\left(i k_{0}-e(\rho)\right)}{\left[i k_{0}-e(\rho)\right]^{2}+4 \pi^{2} T^{2}}\right]\right. \\
& +u\left[k_{0}^{2}+e^{2}(\rho)\right] \frac{(\rho-1)\left[i k_{0}-e(\rho)\right]^{2}+4 \pi^{2} T^{2} \rho}{\left[i k_{0}-e(\rho)\right]^{2}\left(\left[i k_{0}-e(\rho)\right]^{2}+4 \pi^{2} T^{2}\right)} \\
& \left.+\frac{O(T)}{\left[i k_{0}-e(\rho)\right]^{2}+4 \pi^{2} T^{2}}\right\}
\end{aligned}
$$

where $b_{1}=2$ for $G_{1}$ and $b_{2}=1$ for $G_{2}$.

Using these explicit expressions it now easy to check that $F_{1}$ and $F_{2}$ are uniformly bounded by some constant $K$ (independent of $T$ as $T \rightarrow 0$ ). To complete the proof of Lemma 1, there remains to check that these functions $F_{1}$ and $F_{2}$ also decay like any power as $T|\vec{x}| \rightarrow \infty$. For $F_{1}$ there is obviously nothing to check remarking the function $\chi(|\vec{x}| \leq 1)$ in (II.20). Hence we have only to prove

$$
(1+|\vec{x}| T)^{p}\left|F_{2}\right| \leq K_{p}
$$

for some constant $K_{p}$ independent from $T$. Since

$$
(1+|\vec{x}| T)^{p} e^{i \sigma|\vec{x}| \rho}=\left(1-T \frac{i}{\sigma} \frac{d}{d \rho}\right)^{p} e^{i \sigma|\vec{x}| \rho}
$$

we have

$$
\left|(1+|\vec{x}| T)^{p} F_{2}(\vec{x}, t)\right| \leq K_{1} \sup _{\sigma= \pm 1} \sum_{k_{0}} \int_{0}^{\infty} d \rho\left|\left(1+T \frac{i}{\sigma} \frac{d}{d \rho}\right)^{p} G_{2}\left(k_{0}, \rho\right)\right|
$$

where we bounded the factors $\left|f_{i}\right|$ are bounded by constants. Now, performing the change of variable $w=\rho^{2}-1$, using the fact that the $u$ function has compact support, and the fact that the sum over $k_{0}$ is bounded away from 0 since by ([I.2) $\left|k_{0}\right| \geq T$, it is a trivial power counting exercise to check that (II.26) is actually bounded by a constant. 


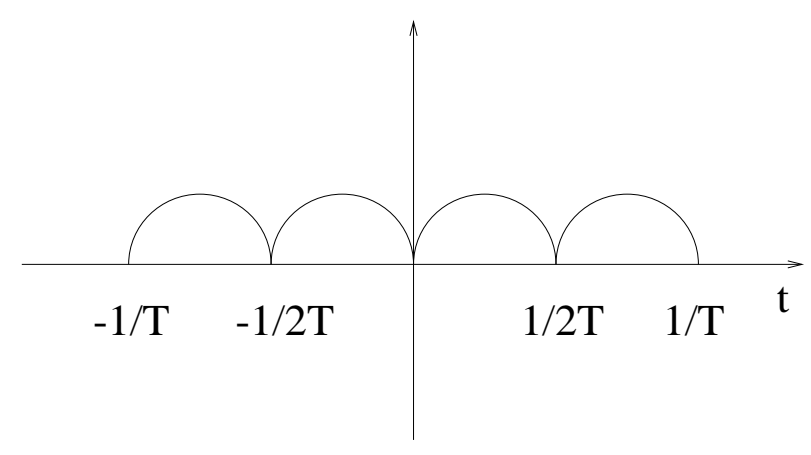

Figure 1: The function $\varepsilon(t) \sin (2 \pi T t)$

Remark that it is not possible to improve significantly Lemma 1. Actually if we try in ([1.7) to obtain e.g. more factors such as $(1+f(t)+|x|)$, identity (II.16) should be applied several times and the action of two or more $\Delta$ operators on the free propagator ([I.3) would generate terms that diverge when $T \rightarrow 0$. Similarly, if the factor $(1+|x|)$ appears more than one time, some corresponding factors $f_{i}$ would not remain bounded when $|x| \rightarrow \infty$.

In the following we will use the spatial decay of the propagator to integrate and the following lemma will be useful

Lemma 2 Let the interval $\left[-\frac{1}{T}, \frac{1}{T}[\right.$ be divided into eight sub-intervals

$$
I_{j}=:\left[-\frac{1}{T}+\frac{j-1}{4 T},-\frac{1}{T}+\frac{j}{4 T}[1 \leq j \leq 8\right.
$$

Then

$$
\frac{1}{1+f(t)+|\vec{x}|} \leq \frac{1}{1+\frac{2}{\pi}\left|t-t_{j}\right|+|\vec{x}|}
$$

where $t_{j}=-\frac{1}{T}+\frac{j-1}{4 T}$ for $j$ even number and $t_{j}=-\frac{1}{T}+\frac{j}{4 T}$ for $j$ odd number.

Proof. Remember that $f(t)=\varepsilon(t) \frac{\sin 2 \pi T t}{2 \pi T}$ is positive and periodic with period $1 / 2 T$ (see Fig.(1).

In each interval $I_{j}$ with $j$ even, the function $\varepsilon(t) \sin 2 \pi T t$ is higher or equal to the line $4 T\left(t-t_{j}\right)$ while for $j$ odd it is higher that the decreasing line $-4 T\left(t-t_{j}\right)$. The proof follows

\footnotetext{
${ }^{1}$ Splitting $C=\sum_{j=1}^{8} C_{j}$ according to which interval we are in, and taking $t_{j}$ as the new origin, we could in fact obviously restrict ourselves to proving the main result of this paper for $j=5$, where $t \geq 0$ and $f(t)$ is simply $t$.
} 


\section{II.4 Slice decomposition}

To introduce multiscale analysis we can work directly in position space. We then write the propagator as

$$
C^{u}(\vec{x}, t)=\sum_{j=0}^{j_{M}+1} C^{j}(\vec{x}, t) ; \quad C^{j}(\vec{x}, t)=C^{u}(\vec{x}, t) \chi_{\Omega_{j}}(\vec{x}, t)
$$

where $\chi_{\Omega}(\vec{x}, t)$ is the characteristic function of the subset $\Omega \subset R^{4}$

$$
\begin{aligned}
\chi_{\Omega}(\vec{x}, t) & =1 \text { if }(\vec{x}, t) \in \Omega \\
& =0 \text { otherwise }
\end{aligned}
$$

and the subset $\Omega_{j}$ is defined as follows:

$$
\begin{aligned}
& \Omega_{j}=\left\{(\vec{x}, t) \mid M^{j-1} \leq(1+|\vec{x}|)^{\frac{3}{4}}(1+f(t)+|\vec{x}|)^{\frac{1}{4}}<M^{j} \quad\right\} 0 \leq j \leq j_{M} \\
& =\left\{(\vec{x}, t) \mid M^{j_{M}} \leq(1+|\vec{x}|)^{\frac{3}{4}}(1+f(t)+|\vec{x}|)^{\frac{1}{4}} \quad\right\} j=j_{M}+1
\end{aligned}
$$

where $M>0$ is a constant that will be chosen later. In Appendix A we discuss why the relative powers $3 / 4$ and $1 / 4$ for $(1+|\vec{x}|)$ and $(1+f(t)+|\vec{x}|)$ are convenient. $j_{M}$ is defined as the temperature scale $M^{j_{M}} \simeq 1 / T$, more precisely

$$
j_{M}=1+I\left[\frac{\ln \left(T^{-1}\right)}{\ln M}\right]
$$

where $I$ means the integer part. With these definitions

$$
\sum_{j=0}^{j_{M}+1} \chi_{\Omega_{j}}(\vec{x}, t)=1 \text {. }
$$

This decomposition is somewhat dual to the usual slice decomposition in momentum space of the renormalization group. Now, for each slice $j$ we can introduce a corresponding lattice decomposition. We work at finite volume $\Lambda:=[-\beta, \beta] \times \Lambda^{\prime}$, where $\Lambda^{\prime}$ is a finite volume in the three dimensional space. For $j \leq j_{M}$ we partition $\Lambda$ in cubes of side $M^{j}$ in all directions, forming the lattice $\mathcal{D}_{j}$. For that we introduce the function

$$
\begin{aligned}
\chi_{\Delta}(x) & =1 & & \text { if } x \in \Delta \\
& =0 & & \text { otherwise }
\end{aligned}
$$

satisfying $\sum_{\Delta \in \mathcal{D}_{j}} \chi_{\Delta}(x)=\chi_{\Lambda}(x)$. For $j=j_{M}+1$ we partition $\Lambda$ in cubes of side $M^{j_{M}}$ in all directions, forming the lattice $\mathcal{D}_{j_{M}+1}=\mathcal{D}_{j_{M}}$. We define the union of all partitions $\mathcal{D}=\cup_{j} \mathcal{D}_{j}$. 
Auxiliary scales The function $\chi_{\Omega_{j}}$ actually mixes temporal and spatial coordinates. In order to sharpen the analysis of $\vec{x}$ and $t$, we will need later an auxiliary slice decoupling for each scale $j$ :

$$
C^{j}(\vec{x}, t)=\sum_{k=0}^{k_{M}(j)} C^{j, k}(\vec{x}, t) ; \quad C^{j, k}(\vec{x}, t)=C^{j}(\vec{x}, t) \chi_{\Omega_{j, k}}(t)
$$

where, for any $j \leq j_{M}$ we defined

$$
\begin{aligned}
\Omega_{j, k} & =\left\{t \mid M^{j+k-1} \leq f(t)<M^{j+k}\right. & & k>0 \\
& =\left\{t \mid 0 \leq f(t)<M^{j}\right. & & k=0
\end{aligned}
$$

and $k_{M}(j)$ is defined as

$$
k_{M}(j)=\min \left\{j_{M}-j, 3 j\right\}
$$

The bound $k \leq j_{M}-j$ is obtained observing that $f(t) \leq M^{j_{M}}$ in any case by periodicity. The bound $k \leq 3 j$ is obtained observing that $(1+f(t))^{\frac{1}{4}} \leq M^{j}$.

The case $j=j_{M}+1$ is special. In this case we must have $0 \leq f(t) \leq M^{j_{M}}$ by periodicity, therefore there is no $k$ decomposition. Actually we say that $k=0$ and we define

$$
\Omega_{j_{M}+1,0}=\left\{t \mid \quad 0 \leq f(t) \leq M^{j_{M}}\right\}
$$

Spatial constraints For any $j$ and $k$ fixed, the spatial decay is constrained too. We must distinguish three cases:

- $j<j_{M}$ and $k>0$ : then there is a non zero contribution only for

$$
M^{j-\frac{k}{3}-\frac{4}{3}} 2^{-\frac{1}{3}} \leq(1+|\vec{x}|) \leq M^{j-\frac{k}{3}+\frac{1}{3}}
$$

- $j \leq j_{M}$ and $k=0$ : then there is a non zero contribution only for

$$
M^{j-\frac{4}{3}} 2^{-\frac{1}{3}} \leq(1+|\vec{x}|) \leq M^{j}
$$

- $j=j_{M}+1$ : then there is a non zero contribution only for

$$
M^{j_{M}} 2^{-\frac{1}{3}} \leq(1+|\vec{x}|)
$$


Power counting and scaled decay of the propagator Now for each $j$ and $k$ we can estimate more sharply the propagator $C^{j k}$. We distinguish three cases:

- for $j<j_{M}$ and $k>0$ we have

$$
\left|C^{j, k}(\vec{x}, t)\right| \leq K_{1} M^{-2 j-\frac{2}{3} k} M^{\frac{7}{3}} 2^{\frac{1}{3}} \chi_{j, k}(\vec{x}, f(t))
$$

where the function $\chi_{j, k}$ is defined by

$$
\begin{array}{rlll}
\chi_{j, k}(\vec{x}, t) & =1 & & \text { if }|\vec{x}| \leq M^{j-\frac{k}{3}+\frac{1}{3}}, f(t) \leq M^{j+k} \\
& =0 & & \text { otherwise }
\end{array}
$$

and the function $F(\vec{x}, t)$ is bounded by $K_{p}$.

- for $j \leq j_{M}$ and $k=0$ we have

$$
\left|C^{j, k}(\vec{x}, t)\right| \leq K_{1} M^{-2 j} M^{\frac{8}{3}} 2^{\frac{2}{3}} \chi_{j, 0}(\vec{x}, f(t))
$$

where the function $\chi_{j, 0}$ is defined by

$$
\begin{aligned}
& \chi_{j, 0}(\vec{x}, t)=1 \quad \text { if }|\vec{x}| \leq M^{j}, f(t) \leq M^{j} \\
& =0 \quad \text { otherwise }
\end{aligned}
$$

- for $j=j_{M}+1$ we have

$$
\left|C^{j_{M}+1,0}(\vec{x}, t)\right| \leq M^{-2 j_{M}} 2^{\frac{2}{3}} \chi_{j_{M}+1,0}(f(t)) \frac{K_{p}}{\left(1+M^{-j_{M}}|\vec{x}|\right)^{p}}
$$

where the function $\chi_{j_{M}+1,0}$ is defined by

$$
\begin{aligned}
& \chi_{j_{M}+1,0}(t)=1 \text { if } f(t) \leq M^{j_{M}} \\
& =0 \text { otherwise }
\end{aligned}
$$

and the spatial decay for $|\vec{x}|$ comes from the decay of the function $F$ in (II.9).

In the following, the multiscale analysis is essentially performed using the $j$ index. The auxiliary structure will be introduced only in section IV. In that 
section we will also need to exchange the sums over $j$ and $k$. The constraints on the maximal value of $k, k_{M}(j)$, are then changed into constraints on $j$ :

$$
\sum_{j=0}^{j_{M}+1} \sum_{k=0}^{k_{M}(j)} C^{j, k}=\sum_{k=0}^{\frac{3 j_{M}}{4}} \sum_{j \in J(k)} C^{j, k}
$$

where

$$
\begin{aligned}
J(k) & =\left[\frac{k}{3}, j_{M}-k\right] \quad \text { for } k>0 \\
J(0) & =\left[0, j_{M}+1\right]
\end{aligned}
$$

\section{II.5 Partition function}

We introduce now the local four point interaction

$$
I(\psi, \bar{\psi})=\lambda \int_{\Lambda} d^{4} x\left(\bar{\psi}_{\uparrow} \psi_{\uparrow}\right)\left(\bar{\psi}_{\downarrow} \psi_{\downarrow}\right)=\lambda \int_{\Lambda} d^{4} x \prod_{c=1}^{4} \psi_{c}
$$

where $\psi_{c}$ is defined as:

$$
\psi_{1}=\bar{\psi}_{\uparrow} \quad \psi_{2}=\psi_{\uparrow} \quad \psi_{3}=\bar{\psi}_{\downarrow} \quad \psi_{4}=\psi_{\downarrow}
$$

The partition function is then defined as

$$
\begin{aligned}
Z_{\Lambda}^{u} & =\int d \mu_{C^{u}}(\psi, \bar{\psi}) e^{I(\psi, \bar{\psi})}=\sum_{n=0}^{\infty} \frac{1}{n !} \int d \mu_{C^{u}}(\psi, \bar{\psi}) I(\psi, \bar{\psi})^{n} \\
& =\sum_{n=0}^{\infty} \frac{1}{n !} \int d \mu_{C^{u}}(\psi, \bar{\psi}) \prod_{v \in V} I_{v}(\psi, \bar{\psi})
\end{aligned}
$$

where $V$ is the set of $n$ vertices and $I_{v}(\psi, \bar{\psi})$ denotes the local interaction at vertex $v$. Now we can introduce slice decomposition over fields:

$$
\psi_{c}=\sum_{j=0}^{j_{M}+1} \psi_{c}^{j}
$$

hence

$$
I_{v}(\psi, \bar{\psi})=\lambda \sum_{J_{v}} \int_{\Lambda} d^{4} x_{v} \prod_{c=1}^{4} \psi_{c}^{j_{c}^{v}}
$$


where $x_{v}$ is the position of the vertex $v, J_{v}=\left(j_{1}^{v}, j_{2}^{v}, j_{3}^{v}, j_{4}^{v}\right)$ gives the slice indices for the fields hooked to $v$. Now we write

$$
I(v)=\lambda \sum_{J_{v}} \sum_{\Delta_{v}} \int_{\Delta_{v}} d^{4} x_{v} \prod_{c=1}^{4} \psi_{c}^{j_{c}^{v}}
$$

where $\Delta_{v} \in \mathcal{D}_{0}$ and

$$
\begin{aligned}
Z_{\Lambda}^{u}= & \sum_{n=0}^{\infty} \frac{\lambda^{n}}{n !} \sum_{J_{V}} \sum_{\Delta_{V}} \\
& {\left[\prod_{v} \int_{\Delta_{v}} d^{4} x_{v}\right] \int d \mu_{C^{u}}(\psi, \bar{\psi})\left[\prod_{v}\left(\prod_{c=1}^{4} \psi_{c}^{j_{c}^{v}}\left(x_{v}\right)\right)\right], }
\end{aligned}
$$

where we denoted any set $\left\{a_{v}\right\}_{v \in V}$ by $a_{V}$.

The Grassmann functional integral at the $n$-th order in (II.57) can be written as a determinant

$$
\int d \mu_{C^{u}}(\psi, \bar{\psi})\left[\prod_{v}\left(\prod_{c=1}^{4} \psi_{c}^{j_{c}^{v}}\left(x_{v}\right)\right)\right]=\operatorname{det} M\left(J_{V},\left\{x_{v}\right\}\right)
$$

where $M\left(J_{V},\left\{x_{v}\right\}\right)$ is a $2 n \times 2 n$ matrix, whose rows correspond to fields and whose columns correspond to antifields. Therefore, for a given vertex $v$, $\psi_{1}\left(x_{v}\right)$ and $\psi_{3}\left(x_{v}\right)$ correspond to columns and $\psi_{2}\left(x_{v}\right)$ and $\psi_{4}\left(x_{v}\right)$ correspond to rows. The matrix element is then

$$
M_{v c ; \bar{v} \bar{c}}=\delta_{j_{c}^{v}, j \overline{\bar{c}}} C^{j_{c}^{v}}\left(x_{v}, x_{\bar{v}}\right)
$$

where $c \in C=:\{2,4\}$ are field indices and $\bar{c} \in \bar{C}=:\{1,3\}$ are antifield indices.

Notations For each cube $\Delta$ we denote by $i_{\Delta}$ its slice index, that is $\Delta \in \mathcal{D}_{j}$ with $j=i_{\Delta}$. We call ancestor of any cube $\Delta \in \mathcal{D}_{j}, \mathcal{A}(\Delta)$, the unique cube $\Delta^{\prime} \in \mathcal{D}_{j+1}$ satisfying $\Delta \subset \Delta^{\prime}$ (see Fig.2). In the same way for any set $S$ of cubes in $\mathcal{D}_{j}$, we call ancestor of $S$ the set $\mathcal{A}(S)=\cup_{\Delta \in S} \mathcal{A}(\Delta)$. We call $\Delta_{v}^{j}$, the unique cube $\Delta \in \mathcal{D}_{j}$, for any $j \geq i_{\Delta_{v}}$, satisfying $\Delta_{v} \subset \Delta$ (for $j=i_{\Delta_{v}}$ we have $\Delta_{v}^{j}=\Delta_{v}$ ). (We remark that for the moment all $i_{\Delta_{v}}=0 \forall \Delta_{v}$ ).

In the following we will denote by $h_{c}^{v}$ the half-line corresponding to the field $\psi_{c}^{j_{c}^{v}}\left(x_{v}\right)$. We say that $h_{c}^{v}$ is external field for the cube $\Delta$ if $\Delta_{v} \subseteq \Delta$, 


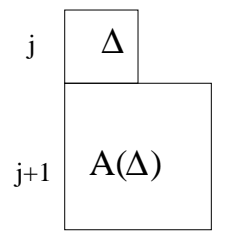

Figure 2: Ancestor

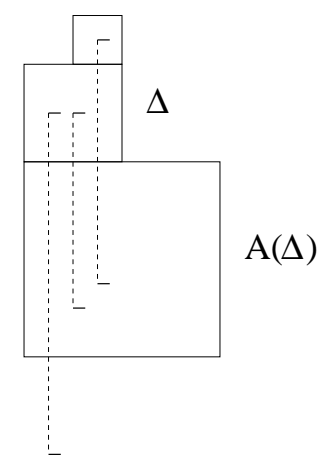

Figure 3: External fields for $\Delta$

$i_{\Delta}<j_{c}^{v}$ and there exist at least one field $h_{c^{\prime}}^{v}$ hooked to $v$ (different from $h_{c}^{v}$ ) with attribution $j_{c^{\prime}}^{v} \leq i_{\Delta}$ (see Fig.3). We call $E(\Delta)$ the set of external fields and antifields of $\Delta$. In the same way we denote by $E(S)=\cup_{\Delta \in S} E(\Delta)$ the set of external fields and antifields of the subset $S \subset \mathcal{D}_{j}$.

We need also to introduce some notations for the fields with smallest index attached to a vertex $v$. We call $i_{v}$ the smallest scale of the vertex $v$, $n_{v}$ the number of fields hooked to $v$ with band index $j=i_{v}\left(1 \leq n_{v} \leq 4\right)$ and $\sigma_{v}$ the set of indices of these $n_{v}$ fields with $j=i_{v}$, which is necessarily non-empty. Finally we distinguish the particular field in $\sigma_{v}$ with lowest value of $c$, which we call $c_{v}$.

$i_{v}=\inf \left\{j_{c}^{v} \mid 1 \leq c \leq 4\right\} ; \sigma_{v}=\left\{c \mid j_{c}^{v}=i_{v}\right\} ; n_{v}=\left|\sigma_{v}\right| ; c_{v}=\inf \left\{c \in \sigma_{v}\right\}$

We say that a vertex $v$ belongs to a cube $\Delta \in \mathcal{D}_{j}$ if $x_{v} \in \Delta$, and we denote the corresponding set of vertices by

$$
V(\Delta)=\left\{v \mid \Delta_{v} \subseteq \Delta\right\}
$$

In the same way we denote by $V(S)=\cup_{\Delta \in S} V(\Delta)$ the set of vertices belonging to the subset $S \subset \mathcal{D}_{j}$. 
We then say that a vertex $v$ is internal for a cube $\Delta \in \mathcal{D}_{j}$ if $v$ belongs to $\Delta$ and $i_{v} \leq j$. The set of internal vertices of $\Delta$ is therefore defined as

$$
I(\Delta)=V(\Delta) \cap\left\{v \mid i_{v} \leq j\right\}
$$

We remark that there may be vertices in $V(\Delta) \backslash I(\Delta))$. In the same way we denote by $I(S)=\cup_{\Delta \in S} I(\Delta)$ the set of internal vertices for the subset $S \subset \mathcal{D}_{j}$. Remark that, if $v \in I(\Delta)$, then $v \in I\left(\Delta^{\prime}\right)$ for any $\Delta^{\prime}$ such that $\Delta \subseteq \Delta^{\prime}$.

\section{Connected functions}

In order to compute physical quantities, we need to extract connected functions. For instance $\mathcal{Z}$ in perturbation theory is the sum over all vacuum graphs corresponding to the full expansion of the determinant in ([1.58), and we know that the logarithm of $\mathcal{Z}$ is the same sum but restricted to connected graphs. But while in ordinary graphs the connectedness can be read directly from the propagators joining vertices, here we need for constructive reasons to test the connection between different cubes in $\mathcal{D}$ by a multiscale cluster expansion. Then the computation of $\log \mathcal{Z}$ is achieved through a Mayer expansion $[R]$.

For this purpose we must introduce two kinds of connections, vertical connections between cubes at adjacent levels $j-1$ and $j$, whose scale is defined as $j$, and horizontal connections between cubes at the same level $j$, whose scale is defined as $j$. (We remark that there is therefore no vertical connection of scale 0 ). The difficulty is that our definition of these connections is inductive, starting from the scale zero towards the scale $j_{M}$.

We define a connected polymer $Y$ as a subset of cubes in $\mathcal{D}$, such that for any two cubes $\Delta, \Delta^{\prime} \in Y$, there exists a chain of cubes $\Delta_{1}, \ldots, \Delta_{N} \in Y$ such that $\Delta_{1}=\Delta, \Delta_{N}=\Delta^{\prime}$ and there is a connection between $\Delta_{i}$ and $\Delta_{i-1}$ for any $i=2, \ldots, N$.

For each scale $j$ we define connected subpolymers at scale $j$ as subsets of cubes belonging to $\cup_{q=0}^{j} \mathcal{D}_{q}$, that are connected through connections of scale $\leq j$. These are the analogs of the quasi-local subgraphs in $[\mathrm{R}]$. As for usual graphs, we call $Y_{k}^{j}(k=1, \ldots, c(j))$ the $c(j)$ connected polymers at scale $j$ and $y_{k}^{j}$ their restriction to $\mathcal{D}_{j}$. The set of external fields for $Y_{k}^{j}$ then corresponds to the set of external fields for $y_{k}^{j}$, which is denoted by $E\left(y_{k}^{j}\right)$.

\section{Connections}




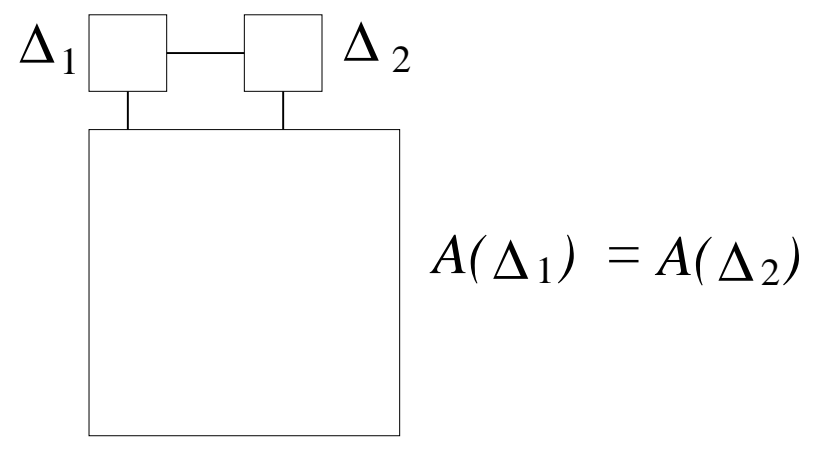

Figure 4: Example of vertical and horizontal connections

1) For any pair $\Delta, \Delta^{\prime}$, with $\Delta, \Delta^{\prime} \in \mathcal{D}_{j}$ and $\Delta \neq \Delta^{\prime}$, we say that there is a horizontal connection, or $h$-connection $\left(\Delta, \Delta^{\prime}\right)$ between them if there exists a propagator $C^{j}\left(x_{v}, x_{v^{\prime}}\right)$ with $\Delta_{v} \subseteq \Delta$ and $\Delta_{v^{\prime}} \subseteq \Delta^{\prime}$ in the expansion of the determinant of ([1.58). (This definition is not inductive).

It is also convenient to introduce generalized notions: a "generalized cube" $\tilde{\Delta}$ of scale $j$ is a subset of cubes of scales $j$ and a generalized horizontal connection, or $g h$-connection $\left(\tilde{\Delta}, \tilde{\Delta}^{\prime}\right)$ is a propagator $C^{j}\left(x_{v}, x_{v^{\prime}}\right)$ with $\Delta_{v} \subseteq \tilde{\Delta}$ and $\Delta_{v^{\prime}} \subseteq \tilde{\Delta}^{\prime}$ in the expansion of the determinant of (II.58).

2) For each connected subpolymer at scale $j$, denoted by $Y$, we suppose by induction that we have defined all subconnections for the subpolymers in $Y$ of scales $\leq j$. We now say that there is a vertical connection between each cube of $y=Y \cap \mathcal{D}_{j}$ and its ancestor if one of the following conditions hold:

- we can associate to $y$ a new internal vertex $v$ in $Y$ that has never been associated previously by the inductive process to any other vertical connection at scale $j^{\prime} \leq j$. (We remark that several vertical connections can be associated to a single vertex, and that vertical connections can form loops (see Fig.(1)). In this case the connection is called a $v$ connection. The set of all such $v$-connections for a fixed $y$ and a fixed new internal vertex $v$ is called the $v$-block associated to the vertex $v$.

- there is no such new internal vertex $v$ for $y$, but $|E(y)|>0$. In this case the connection is called an $f$-connection, and $|E(y)|$ is called the strength of the connection. The set of all such $f$-connections for a fixed set of external lines is called the $f$-block associated to these external lines. 


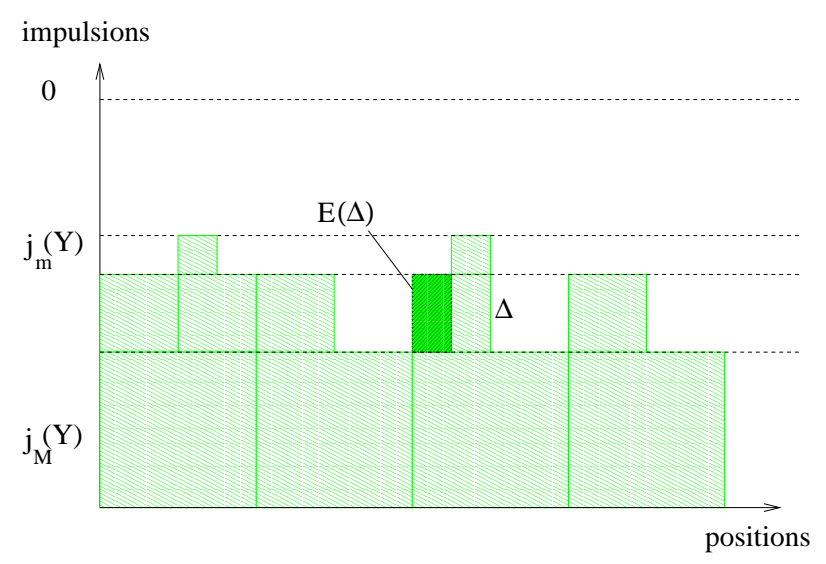

Figure 5: An example of polymer $Y$.

In fact in this paper we will restrict ourselves to the analysis and bound for connected subpolymers for which in the second case, we always have $|E(y)| \geq 6$, since the other cases need renormalization.

When there is no vertical connection, i.e. no new vertex, and $|E(y)|=0$, we call $Y$ simply a (vacuum) polymer.

\section{III.1 Polymer structure}

With these definitions in phase space (in our usual representation, for which index space is vertical) all polymers have a "solid on solid" profile (see Fig.5)].

We define the highest and lowest slice index of each polymer $Y$ as

$$
\begin{aligned}
& m_{Y}=\min _{\Delta \in Y} i_{\Delta} \\
& M_{Y}=\max _{\Delta \in Y} i_{\Delta} .
\end{aligned}
$$

For each cube $\Delta \in Y$, we define the "exposed volume of $\Delta$ " as

$$
\operatorname{Ex}(\Delta)=\cup_{\left\{\begin{array}{c}
\Delta^{\prime} \in \mathcal{D} \text { with } \\
\Delta=\mathcal{A}\left(\Delta^{\prime}\right) \text { and } \Delta^{\prime} \notin Y
\end{array}\right\}} \Delta^{\prime}
$$

In other words this is the part of $\Delta$ that contains no other cube of $Y$, and is therefore at the upper border of the polymer (see Fig.5). An element $\Delta \in Y$ is called a "summit cube" if $\operatorname{Ex}(\Delta) \neq \emptyset$, and we define the "border of $Y$ ",

\footnotetext{
${ }^{2}$ This is not the unique possible choice. In [AR1] polymers with holes or overhangs are allowed. Here we choose polymers without holes for simplicity.
} 
$B(Y)$, as the union of all summit cubes: $E(Y)=\cup_{\{\Delta \mid E x(\Delta) \neq \emptyset\}} \Delta$. We remark that $\{\operatorname{Ex}(\Delta)\}_{\Delta \in B(Y)}$ is a partition of the volume occupied by $Y$, and the sum over $\Delta_{v}$ for any $v$ in $Y$ can be written as

$$
\sum_{\Delta_{v} \in \mathcal{D}_{0}} \int_{\Delta_{v}} d x_{v}=\sum_{\Delta_{v} \in B(Y)} \int_{E x\left(\Delta_{v}\right)} d x_{v} .
$$

and we say that the vertex $v$ is localized in the summit cube $\Delta_{v} \in B(Y)$.

Trees and Forests The connections among cubes in a polymer are the constructive analogs of lines in a graph. It is useful to select among these connections a minimal set i.e. a tree connecting the cubes of the polymer. This is the purpose of the expansion defined below. But we perform this task in two steps. In the main step, called the multiscale cluster expansion, we select vertices, external lines and propagators which form $v$-blocks, $f$ -

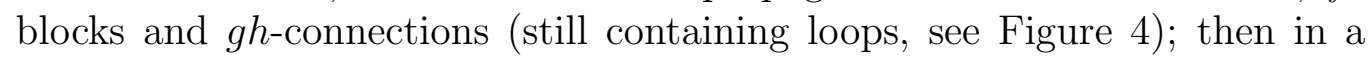
second, auxiliary step, called the tree and root selection, we eliminate some redundant connections from the $v$-blocks and $f$-blocks, and we localize the $g h$-connections into ordinary $h$-connections, in order to obtain an ordinary tree connecting all cubes of the polymer; moreover we select for any subpolymer a particular cube called the root, in a coherent way.

Just like the definition of the connections, our expansion is inductive. The multiscale expansion starts from the slices with lowest index towards the ones with higher index. The tree and root selection works also inductively but in the inverse order, from the slices with highest index towards the ones with lower index.

In the end the particular connections which are selected by the expansion to form the tree will be called links (more precisely $v$-link, $f$-link, or $h$-link, if they correspond to a $v$-connection, an $f$-connection, or an $h$-connection).

Therefore by construction for each subpolymer $Y_{k}^{j}$, the set of horizontal and vertical links of scale $j^{\prime} \leq j$ forms a subtree $\mathcal{T}_{j}$ spanning the subpolymer; and for the union $\cup_{k} Y_{k}^{j}$ of subpolymers at scale $j$ it forms a forest $\mathcal{F}_{j}$ (i.e. a set of disjoint trees).

The forest $\mathcal{F}_{j}$ at scale $j$ is built from the forest $\mathcal{F}_{j-1}$ at scale $j-1$, by adding a set of $v$-links or $f$-links of scale $j-1$ and a set of $h$-links of scale $j$. Therefore $\mathcal{F}_{0} \subset \mathcal{F}_{1} \subset \ldots \subset \mathcal{F}_{j_{M}+1}:=\mathcal{F}$ (such a growing sequence of forests is technically called a "jungle" [AR2]). 


\section{III.2 Multiscale Cluster Expansion}

In this first step we build connected polymers by choosing $v$-blocks, $f$-blocks and $g h$-links which ensure the connectedness of the polymer. This is done through Taylor expansions with integral remainders, inductively from scale 0 to scale $j_{M}$.

We build the connected subpolymers at scale $j+1$, knowing already the connected subpolymers at scales $j^{\prime}<j+1$. We perform first the vertical expansion, then the horizontal one, except for the first slice, for which we start with the horizontal one.

\section{III.2.1 Vertical expansion}

For each connected subpolymer $Y_{k}^{j}$, we define $I_{j}\left(Y_{k}^{j}\right)$ as the subset of internal vertices that have been selected until the step $j$. We can also define the union of all vertices already selected at scale $j: I_{j}\left(\mathcal{F}_{j}\right)=\cup_{k} I_{j}\left(y_{k}^{j}\right)$. We extract first the $v$-blocks, then the $f$-blocks of scale $j+1$ (all other connections at scale $j^{\prime} \leq j$ being already fixed).

$v$-blocks First we test the existence of a $v$-block associated to a vertex. We want therefore to know whether $I\left(y_{k}^{j}\right) \backslash I_{j}\left(y_{k}^{j}\right) \neq \emptyset$ for each $y_{k}^{j}$, namely whether there is at least one internal vertex $v$ that has not already been selected,

For this purpose we introduce into (โI.57) the identity

$$
1=\prod_{v \in V \backslash I_{j}\left(\mathcal{F}_{j}\right)}\left[\prod_{c=1}^{4}\left(v^{j}\left(j_{c}^{v}\right)+v^{>j}\left(j_{c}^{v}\right)\right)\right]
$$

where we defined

$$
\begin{aligned}
v^{j}\left(j_{c}^{v}\right) & =1 \quad \text { if } \quad j_{c}^{v} \leq j \\
& =0 \quad \text { otherwise }
\end{aligned}
$$

and $v^{>j}\left(j_{c}^{v}\right)=1-v^{j}\left(j_{c}^{v}\right)$. Remark that $v$ is internal vertex for $\Delta_{v}^{j}$ if there is at least one field hooked to $v$ with $j_{c}^{v} \leq j$. Therefore, to select one new internal vertex for $y_{j}^{k}$ we define the function

$$
F\left(w_{y_{k}^{j}}^{\prime}\right)=\prod_{v \in V(y) \backslash I_{j}(y)} \prod_{c=1}^{4}\left[\left(w_{y_{k}^{j}}^{\prime} v^{j}\left(j_{c}^{v}\right)+v^{>j}\left(j_{c}^{v}\right)\right)\right]
$$


The identity (III.66) corresponds to $F\left(w_{y_{k}^{j}}^{\prime}=1\right)$. Now we apply the first order Taylor formula:

$$
F(1)=F(0)+\int_{0}^{1} d w_{y_{k}^{j}}^{\prime} F^{\prime}\left(w_{y_{k}^{j}}^{\prime}\right)
$$

where

$$
F(0)=\prod_{v \in V\left(y_{k}^{j}\right) \backslash I_{j}\left(y_{k}^{j}\right)}\left[\prod_{c=1}^{4} v^{>j}\left(J_{v}\right)\right]
$$

means there is no new internal vertex for $y_{j}^{k}$ (hence $I_{j}\left(y_{j}^{k}\right)=I\left(y_{j}^{k}\right)$ ), and we must go to the next paragraph to test for the existence of external fields ( $f$-blocks). On the other hand, the integral remainder

$$
\begin{gathered}
F^{\prime}\left(w_{y_{k}^{j}}^{\prime}\right)=\sum_{\substack{v \in V\left(y_{k}^{j}\right) \backslash I_{j}\left(y_{k}^{j}\right) \\
\alpha_{v}=1}}^{4} v^{j}\left(j_{c}^{v}\right) \int_{0}^{1} d w_{y_{k}^{j}}^{\prime} \prod_{c^{\prime} \neq \alpha_{v}}\left(w_{y_{k}^{j}}^{\prime} v^{j}\left(j_{c^{\prime}}^{v}\right)+v^{>j}\left(j_{c^{\prime}}^{v}\right)\right) \\
\prod_{\substack{v^{\prime} \in V\left(y_{k}^{j} \backslash I_{j}\left(y_{k}^{j}\right) \\
v^{\prime} \neq v\right.}}\left[\prod_{c=1}^{4}\left(w_{y_{k}^{j}}^{\prime} v^{j}\left(j_{c}^{v^{\prime}}\right)+v^{>j}\left(j_{c}^{v^{\prime}}\right)\right)\right] .
\end{gathered}
$$

extracts one new internal vertex for $y_{j}^{k}$, choosing the field with $c=\alpha_{v}$ to have $j_{c}^{v} \leq j$. To simplify this expression we define

$$
\Upsilon_{j}(v, c)=\left(w_{y_{v}^{\prime}}^{\prime} v^{j}\left(j_{c}^{v}\right)+v^{>j}\left(j_{c}^{v}\right)\right)
$$

Hence the remainder term is written

$$
F^{\prime}\left(w_{y_{k}^{j}}^{\prime}\right)=\sum_{v \in V\left(y_{k}^{j}\right) \backslash I_{j}\left(y_{k}^{j}\right)} \sum_{\alpha_{v}=1}^{4} v^{j}\left(j_{c}^{v}\right) \int_{0}^{1} d w_{y_{k}^{j}}^{\prime} \prod_{\substack{c^{\prime} \neq \alpha_{v} \\ c^{\prime}}} \Upsilon_{j}\left(v, c^{\prime}\right) \prod_{\substack{v^{\prime} \in V\left(y_{k}^{j}\right) \backslash I_{j}\left(y_{k}^{j}\right) \\ v^{\prime} \neq v}} \prod_{c=1}^{4} \Upsilon_{j}\left(v^{\prime}, c\right) .
$$

When this remainder term is selected, we have built the $v$-block corresponding to $y_{k}^{j}$ and to the vertex $v$.

This analysis is performed for each connected component $y_{j}^{k}$ before going on. 
$f$-blocks If $w_{y_{k}^{j}}^{\prime}=0$, that is $I\left(y_{k}^{j}\right) \backslash I_{j}\left(y_{k}^{j}\right)=\emptyset$, there is no $v$-block connecting $y_{k}^{j}$ to its ancestor, therefore we must test for the existence of external fields (f-block).

Fo each $v \in I\left(y_{k}^{j}\right)$ (actually in this case $I_{j}\left(y_{k}^{j}\right)=I\left(y_{k}^{j}\right)$ ) we can write the sum over field attributions as follows

$$
\sum_{J_{v}}=\sum_{n_{v}, \sigma_{v}} \sum_{i_{v} \in I_{v}} \sum_{J_{v}^{\prime}}
$$

where we recall that $i_{v}=\min \left\{j_{c}^{v} \mid c=1, \ldots, 4\right\}, \sigma_{v}$ gives the indices of the fields with $j_{c}^{v}=i_{v}$ and $n_{v}=\left|\sigma_{v}\right|$ (【.60). The attribution $i_{v}$ can belong only to the interval $I_{v}=\left[0, l_{v}\right]$ where $l_{v}$ is the scale where the vertex $v$ has been associated to a vertical block. Remark that $l_{v} \leq j-1$ because this vertex has been extracted as internal vertex for some $y_{k^{\prime}}^{j^{\prime}}$ with $j^{\prime}<j$. Finally $J_{v}^{\prime}$ gives the band indices for the $4-n_{v}$ fields that do not belong to the band $i_{v}$ : $j_{c}^{v}>i_{v}, \forall c \notin \sigma_{v}$. Remark that if the field $c=\alpha_{v}$ does not belong to $\sigma_{v}$ then it satisfies the constraint $i_{v}<\alpha_{v} \leq l_{v} \leq j-1$. The interpolating function $F$ is now

$$
F\left(w_{y_{k}^{j}}^{\prime \prime}\right)=\prod_{v \in I\left(y_{k}^{j}\right)} \prod_{\substack{c \notin \sigma v \\ j_{c}^{v}>j}} w_{y_{k}^{j}}^{\prime \prime}
$$

We want to extract external lines until we have convergent power counting. Since in this theory two and four point functions a priori require renormalization [FT1-2], we push the Taylor formula in $w^{\prime \prime}$ to sixth order:

$$
F\left(w^{\prime \prime}=1\right)=\sum_{p=0}^{5} F^{(p)}\left(w^{\prime \prime}=0\right)+\int_{0}^{1} d w^{\prime \prime} F^{(6)}\left(w^{\prime \prime}\right)
$$

where all terms with $p$ odd are zero by parity and the term $F^{(p)}\left(w^{\prime \prime}=0\right)$ for $p=0,2,4$ corresponds to the case of 0,2 and 4 external fields. Finally the integral remainder corresponds to the case of 6 external legs or more. When a field is derived by the Taylor formula at scale $j$, hence is chosen as external field, its band attribution is constrained to the set $j_{c}^{v}>j$. The highest band is constrained to $i_{v} \leq j$, but this was already true because external fields only hook to vertices that have been extracted at some level $j^{\prime} \leq j$ (therefore $\left.i_{v} \leq j-1\right)$.

Remark that the same field may be chosen as external field at different scales. 
When any term in (III.76) is selected except the one with $p=0$ we build the $f$-block corresponding to $y_{k}^{j}$ and to the corresponding set of selected external lines, and we say that this $f$-block has a corresponding strength of $p=2,4$, or 6 .

This analysis is again performed for each connected component $y_{j}^{k}$ before going on.

\section{III.2.2 Horizontal expansion}

The extraction of the vertical blocks has fixed a certain set of generalized cubes at scale $j+1$, called $\tilde{\mathcal{D}}_{j+1}$. The elements of $\tilde{\mathcal{D}}_{j+1}$ are the connected components at scale $j+1$, taking into accounts all previous connections, that is the connections of scale $j^{\prime} \leq j$ and the vertical connections of the $v$ and $f$-blocks of scale $j+1$ that have just been built.

In order to complete the construction of the connected subpolymers at scale $j+1$, we must test horizontal connections between these generalized cubes, that is $g h$-connections. Extracting these $g h$-connections actually corresponds to extracting forests made of such $g h$-connections at scale $j+1$ over these generalized cubes. We denote such a forest by $\mathcal{F}_{j+1}^{h}$. This is done using a so called forest formula.

Forest formula To simplify notation we work at scale $j$ instead of $j+1$. Forest formulas are Taylor expansions with integral remainders which test connections (here the $g h$-connections at scale $j$ ) between $n \geq 1$ points (here the generalized cubes at scale $j$ ) and stop as soon as the final connected components are built. The result is a sum over forests, a forest being a set of disjoint trees.

We use the unordered Brydges-Kennedy Taylor formula, which states [AR2] that for any smooth function $H$ of the $n(n-1) / 2$ variables $u_{l}, l \in$

\footnotetext{
${ }^{3}$ In part II of this study we plan to perform renormalization of the two point function and to simply bound logarithmic divergences such as those of the 4-point function using the smallness of the coupling constant like in [DR2]. For that purpose we need to complicate slightly this definition, and to introduce holes in the vertical direction of our polymers when $f$-blocks have strength 2 or 4 . These complications are not necessary here so we postpone them to this future publication.
} 


$$
\begin{aligned}
P_{n}=\{(i, j) \mid i, j \in\{1, . ., n\}, i \neq j\}, & \\
& \left.H\right|_{h_{l}=1}=\sum_{u-\mathcal{F}} \prod_{q=1}^{k}\left(\int_{0}^{1} d w_{q}\right)\left(\prod_{q=1}^{k} \frac{\partial}{\partial h_{l_{q}}} H\right)\left(h_{l}^{\mathcal{F}}\left(w_{q}\right), l \in P_{n}\right)
\end{aligned}
$$

where $u-\mathcal{F}$ is any unordered forest, made of $0 \leq k \leq n-1$ lines $l_{1}, \ldots, l_{k}$ over the $n$ points. To each line $l_{q} q=1, \ldots, k$ of $\mathcal{F}$ is associated the parameter $w_{q}$, and to each pair $l=(i, j)$ is associated the weakening factor $h_{l}^{\mathcal{F}}\left(w_{q}\right)$. These factors replace the variables $u_{l}$ as arguments of the derived function $\prod_{q=1}^{k} \frac{\partial}{\partial h_{l_{q}}} H$ in (III.77). These weakening factors $h_{l}^{\mathcal{F}}(w)$ are themselves functions of the parameters $w_{q}, q=1, \ldots, k$ through the formulas

$$
\begin{aligned}
h_{i, i}^{\mathcal{F}}(w)= & 1 \\
h_{i, j}^{\mathcal{F}}(w)= & \inf _{l_{q} \in P_{i, j}^{\mathcal{F}}} w_{q}, \quad \text { if } i \text { and } j \text { are connected by } \mathcal{F} \\
& \text { where } P_{i, j}^{\mathcal{F}} \text { is the unique path in the forest } \mathcal{F} \text { connecting } i \text { to } j \\
h_{i, j}^{\mathcal{F}}(w)= & 0 \quad \text { if } i \text { and } j \text { are not connected by } \mathcal{F} .
\end{aligned}
$$

In our case, the $H$ function is the determinant, $P_{n}$ is the set of pairs of generalized cubes at scale $j$

$$
P_{n}=\left\{\left(\tilde{\Delta}, \tilde{\Delta}^{\prime}\right) \mid \tilde{\Delta}, \tilde{\Delta}^{\prime} \in \tilde{\mathcal{D}}_{j}\right\}
$$

We apply the forest formula (III.77) at scale $j$ and we denote the corresponding forest by $\mathcal{F}_{j}^{h}$. Therefore the interpolation parameter $h_{\tilde{\Delta} \tilde{\Delta}^{\prime}}^{\mathcal{F}^{h}}$ is inserted besides the matrix element defined in ([I.59):

$$
M_{v c ; \bar{v} \bar{c}}=\delta_{j_{c}^{v}, j \overline{\bar{c}}}\left[C_{\Delta_{v}^{j}, \Delta_{\bar{v}}^{j}}^{j}\left(x_{v}, x_{\bar{v}}\right)\right]_{j=j_{c}^{v}}
$$

where we defined

$$
C_{\Delta_{v}^{j}, \Delta_{\bar{v}}^{j}}^{j}\left(x_{v}, x_{\bar{v}}\right)=: \chi_{\Delta_{v}^{j}}\left(x_{v}\right) C^{j}\left(x_{v}, x_{\bar{v}}\right) \chi_{\Delta_{\bar{v}}^{j}}\left(x_{\bar{v}}\right)
$$

and $\chi_{\Delta}(x)$ is the characteristic function of $\Delta$, defined by: $\chi_{\Delta}(x)=1$ if $x \in \Delta$ and $\chi_{\Delta}(x)=0$ otherwise. The interpolated matrix element, for any $j_{c}^{v}=j$ is then 


$$
M_{v c ; \bar{v} \bar{c}}\left(h_{\tilde{\Delta}_{c}, \tilde{\Delta}^{\prime}}^{j^{v}}\right)=\delta_{j_{c}^{v}, j \overline{\bar{v}}}\left[h_{\tilde{\Delta}_{v}^{j}, \tilde{\Delta}_{\bar{v}}^{j}}^{j} C_{\Delta_{v}^{j}, \Delta_{\bar{v}}^{j}}^{j}\left(x_{v}, x_{\bar{v}}\right)\right]_{j=j_{c}^{v}}
$$

where we defined $\tilde{\Delta}_{v}^{j}$ as the unique generalized cubes containing $\Delta_{v}^{j}$, and write for simplicity $h_{\tilde{\Delta}_{v}^{j}, \tilde{\Delta}_{\bar{v}}^{j}}^{j}$ instead of $h_{\tilde{\Delta}_{v}^{j}, \tilde{\Delta}_{\bar{v}}^{j}}^{\mathcal{F}_{\bar{v}}^{h}}$.

\section{III.3 Tree and root selection}

Localization of the $g h$-connections We now fix, for each field $h$ or antifield $\bar{h}$ hooked to a vertex $v$, whether it belongs or not to a propagator derived by the horizontal expansions (since this costs only a factor 2 per field or antifield, hence a factor 16 per vertex). As we know the position of $\Delta_{v}$ for any $v$, we know exactly for each $\tilde{\Delta}$ in $y_{k}^{j}$ the set of $h, \bar{h}$ that form at scale $j$ (as $j_{h}^{b}=j$ ) the propagators of the tree $T_{j k}$. We denote this set by $b(\tilde{\Delta})$.

The first, rather trivial step, consists in replacing each $g h$-connection between generalized cubes by an ordinary $h$-link between ordinary cubes. This means, in the propagator $\chi_{\tilde{\Delta}} C^{j} \chi_{\tilde{\Delta}^{\prime}}$ corresponding to the $g h$-connection, that we expand the characteristic functions as $\chi_{\tilde{\Delta}}=\sum_{\Delta \in \mathcal{D}_{j}, \Delta \subset \tilde{\Delta}} \chi_{\Delta}$, and $\chi_{\tilde{\Delta}^{\prime}}=\sum_{\Delta^{\prime} \in \mathcal{D}_{j}, \Delta^{\prime} \subset \tilde{\Delta}^{\prime}} \chi_{\Delta^{\prime}}$. Accordingly the $g h$-connection is localized into an ordinary connection, or $h$-link between $\Delta$ and $\Delta^{\prime}$ t.

Choice of the roots Remember that at each scale $j$ each connected subpolymer $y_{k}^{j}$ is actually made of a set of disjoint generalized cubes $\tilde{\Delta}$. We want now to choose one generalized cube $\tilde{\Delta}_{\text {root }}$ in each $y_{k}^{j}$, called the root of the subpolymer, and one particular cube $\Delta_{\text {root }}$ in each generalized cube $\tilde{\Delta}$ called the root of the generalized cube.

The root cube in $\tilde{\Delta}_{\text {root }}$ is special: it will correspond to the root cube of the whole subpolymer, therefore we will denote it by $\Delta_{\tilde{\sim}}^{0}$ root .

Finally, in each $y_{k}^{j}$, for each $\tilde{\Delta} \neq \tilde{\Delta}_{\text {root }}$, we want to choose one field or antifield in $b(\tilde{\Delta})$ as the one contracting towards the root in $T_{j k}$ and we call it $h_{\text {root }}$ (the vertex to which it is hooked being called $v_{\text {root }}$ ). We call then $R_{\text {root }}$ the set of all $h_{\text {root }}$ for all generalized cubes at all the different scales.

\footnotetext{
${ }^{4}$ The corresponding sums are bounded below in two steps: in the first step, at the beginning of section III.4, the set $b$ of the fields for the $h$-links is chosen (and paid in section IV.7.3), and in section IV.6 the contraction between these fields is performed (construction of $T_{j k}$ ). Since in section III.4 the position of all the fields is known, together these two steps pay for the localization of $g h$-connections into ordinary connections.
} 
Remark that the choice of the set $R_{\text {root }}$ can be performed only after the choice of $\tilde{\Delta}_{\text {root }}$. The set of remaining fields in $b(\tilde{\Delta})$ is denoted by $l_{b}(\tilde{\Delta})$ (and called the leaves for $\tilde{\Delta}$ ). Remark that for $\tilde{\Delta}_{\text {root }}$ all fields are leaves: $b(\tilde{\Delta})=l_{b}(\tilde{\Delta})$.

The roots are chosen inductively scale by scale, from bottom up, starting by the biggest index scale $M_{Y}$ of the polymer and going up until the smallest index $m_{Y}$, To break translation invariance, we need to assume from now on that the polymer $Y$ contains a particular point, namely the origin $x=0$.

At the biggest scale we have only one connected component, that must contain the origin $x=0$. Therefore we choose $\tilde{\Delta}_{\text {root }}$ as the unique $\tilde{\Delta}$ containing $x=0$, and $\Delta_{\text {root }}=\Delta_{\text {root }}^{0}$ as the unique cube $\Delta \in \tilde{\Delta}_{\text {root }}$ containing $x=0$. Now for each $\tilde{\Delta} \neq \tilde{\Delta}_{\text {root }}$ we define $\Delta_{\text {root }}$ as the (necessarily unique) cube $\Delta \in \tilde{\Delta}$ containing a field $h_{\text {root }} \in R_{\text {root }}$ of that scale.

With these definitions we can introduce the general inductive rule. We assume that all $\Delta_{\text {root }}$ and $\tilde{\Delta}_{\text {root }}$ have been defined until the scale $j$. We now want to define the roots at scale $j-1$.

Remark that each connected component $y_{k}^{j-1}$ actually corresponds to some generalized cube $\tilde{\Delta}_{0}$ at scale $j$. We denote by $\Delta_{0}$ its root cube. Now we distinguish two cases:

- there exists a cube $\Delta_{1} \in y_{k}^{j-1}$ with $\Delta_{1} \subseteq \Delta_{0}$ which contains either 0 or one $h_{\text {root }}$ at some scale $j^{\prime} \geq j$. Remark that this $\Delta_{1}$ must be unique. Then we define as $\tilde{\Delta}_{\text {root }}$ for $y_{k}^{j-1}$ the unique $\tilde{\Delta}$ with $\Delta_{1} \subseteq \tilde{\Delta}$. Now for all $\tilde{\Delta} \neq \tilde{\Delta}_{\text {root }}$ we introduce $h_{\text {root }}$ and $\Delta_{\text {root }}$ exactly as in the case of the lowest band $M_{Y}$. Finally for $\tilde{\Delta}_{\text {root }}$ we choose $\Delta_{1}$ as root cube: $\Delta_{1}=\Delta_{\text {root }}^{0}$.

- there is no cube $\Delta_{1} \in y_{k}^{j-1}$ with $\Delta_{1} \subseteq \Delta_{0}$ with $0 \in \Delta_{1}$ or $\Delta_{v_{\text {root }}} \subseteq \Delta_{1}$ for some $h_{\text {root }}$ at a lower scale. Therefore we choose as root one of the $\tilde{\Delta} \in y_{k}^{j-1}$ satisfying $\tilde{\Delta} \cap \Delta_{0} \neq \emptyset$ (remark that there must be at least one of such $\tilde{\Delta}$ by construction). For all $\tilde{\Delta} \neq \tilde{\Delta}_{\text {root }}$ we introduce $h_{\text {root }}$ and $\Delta_{\text {root }}$ exactly as in the case of the lowest band $M_{Y}$. Finally for $\tilde{\Delta}_{\text {root }}$ we choose as $\Delta_{\text {root }}^{0}$ one of the cubes satisfying $\Delta \subseteq \Delta_{0}$ (there must be at least one by construction).

For an example see Fig.6, where cubes of three scales are shown. The lines connecting two cubes are are $h$-links. The union $\Delta_{1} \cup \Delta_{2} \cup \Delta_{3}$ is a generalized cube at scale $j$ (corresponding to $\tilde{\Delta}_{0}$ above). From the figure one 


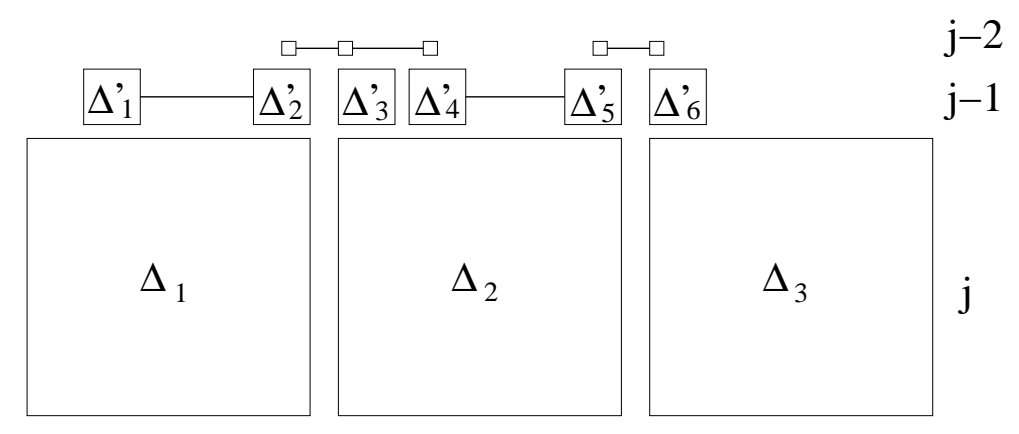

Figure 6: Construction of roots

can see that there are three generalized cubes at scale $j-1$ :

$$
\begin{aligned}
& \tilde{\Delta}_{1}=\Delta_{1}^{\prime} \\
& \tilde{\Delta}_{2}=\Delta_{2}^{\prime} \cup \Delta_{3}^{\prime} \cup \Delta_{4}^{\prime} \\
& \tilde{\Delta}_{3}=\Delta_{5}^{\prime} \cup \Delta_{6}^{\prime}
\end{aligned}
$$

Now, let us say that $\tilde{\Delta}_{0}$ is a root at scale $j, \Delta_{2}$ is the corresponding root cube and $0 \notin \Delta_{2}$ and no $h_{\text {root }}$ has vertex in $\Delta_{2}$. Then we have two equivalent choices for $\tilde{\Delta}_{\text {root }}^{\prime}$ as $\tilde{\Delta}_{2}^{\prime} \cap \Delta_{2} \neq \emptyset$ and $\tilde{\Delta}_{3}^{\prime} \cap \Delta_{2} \neq \emptyset$. Let us take $\tilde{\Delta}_{\text {root }}^{\prime}=\tilde{\Delta}_{2}^{\prime}$. Now inside $\tilde{\Delta}_{2}^{\prime}$ we have again two equivalent choices for $\Delta_{\text {root }}^{\prime}$ as $\Delta_{3}^{\prime}$ and $\Delta_{4}^{\prime} \subset \Delta_{2}$.

Choice of the $v$-links and $f$-links Remember that in order to avoid loops, each time several cubes in $y_{k}^{j}$ have the same ancestor we must choose only one of them in the block to bear a link (either of $v$ or $f$ type). The choice of this cube is completely arbitrary (for instance choose the first ones in some lexicographic ordering of the cubes), except for one constraint. Actually, for each connected subpolymer $y$ the root cube $\Delta_{\text {root }}^{0}$ acts as root for $y$, therefore we decide to always choose as vertical link $\left(\Delta_{\text {root }}^{0}, \mathcal{A}\left(\Delta_{\text {root }}^{0}\right)\right)$. All other choices are arbitrary. This constraint is useful because in the following all the vertical power counting for $y_{k}^{j}$ will be concentrated on this special vertical link $(\Delta, \mathcal{A}(\Delta))\left(\Delta=\Delta_{\text {root }}^{0}\right)$.

At the end of this selection process we have therefore an ordinary tree of either $v, f$ or $h$ links connecting together all cubes of $Y$. 


\section{III.4 Result of the expansion}

As a result of this inductive process we obtain the following expression

$$
\begin{aligned}
& Z_{\Lambda}^{u}=\sum_{n=0}^{\infty} \frac{\lambda^{n}}{n !} \sum_{\Delta_{V}} \sum_{\mathcal{F}} \sum_{V_{d}, \alpha_{V_{d}}} \sum_{a, b, R} \sum_{l_{V_{d}}} \sum_{\left\{J_{h}^{a}\right\},\left\{J_{h}^{a}\right\}} \sum_{\left\{j_{h}^{b}\right\},\{j} \sum_{\left.C_{h}\right\}} \varepsilon_{\mathcal{F}}\left[\prod_{v} \int_{\Delta_{v}} d^{4} x_{v}\right] \\
& {\left[\prod_{j=0}^{j_{M}+1}\left(\prod_{l \in h L_{j}} \int_{0}^{1} d w_{l}\right)\right]\left[\prod_{j=1}^{j_{M}+1}\left(\prod_{l \in v L_{j}} \int_{0}^{1} d w_{l}^{\prime}\right)\right]\left[\prod_{j=1}^{j_{M}+1}\left(\prod_{l \in f L_{j}^{6}} \int_{0}^{1} d w_{l}^{\prime \prime}\right)\right]} \\
& {\left[\prod_{j=0}^{j_{M}+1}\left(\prod_{l \in h L_{j}} C_{\Delta_{l} \bar{\Delta}_{l}}^{j}\left(x_{l}, \bar{x}_{l}\right)\right)\right]\left[\prod_{v \in V_{d}}\left(\sum_{n_{v} \sigma_{v} \rho_{v}} \sum_{i_{v} \in I_{v}} \sum_{J_{v}^{\prime}}\right)\right]\left[\prod_{v \in \bar{V}_{d}} \sum_{J_{v}}\right]} \\
& {\left[\prod_{v \in V_{d}}\left(v^{>j_{m}(v)}\left(j_{\alpha_{v}}^{v}\right) v^{l_{v}}\left(j_{\alpha_{v}}^{v}\right) \prod_{j=0}^{l_{v}-1} \Upsilon_{j}\left(v, \alpha_{v}\right)\right)\right]} \\
& {\left[\prod_{v \in V_{d}} \prod_{c \neq \alpha_{v}}\left(v^{>j_{m}(v)}\left(j_{c}^{v}\right) \prod_{j=0}^{l_{v}} \Upsilon_{j}(v, c)\right)\right]} \\
& {\left[\prod_{v \in \bar{V}_{d}} \prod_{c=1}^{4}\left(v^{>j_{m}(v)}\left(j_{c}^{v}\right) \prod_{j=0}^{j_{M}} \Upsilon_{j}(v, c)\right)\right]} \\
& {\left[\prod_{v \in V_{d}} \prod_{c \notin \sigma_{v}}\left(\prod_{j=0}^{j_{c}^{v}-1} s_{j}(v, c)\right)\right] \operatorname{det} M^{\prime}\left(\left\{w_{l}\right\}\right)}
\end{aligned}
$$

where

- $V_{d}=\{v \in V \mid \exists$ one $v$-link associated to $v\}$ and $\bar{V}_{d}=V \backslash V_{d}$;

- $a=\left\{h_{c}^{v} \mid v \in V_{d}\right.$ and $h_{c}^{v}$ is associated to some $f$-links at one or several scales $\}$;

- $b=\left\{h_{c}^{v} \mid h_{c}^{v}\right.$ is associated to one h-link $\}$;

- $R=R_{\text {root }}=\left\{h_{c}^{v} \mid h_{c}^{v}\right.$ is a root field or antifield $\}$;

- $l_{V_{d}}=\left\{l_{v} \mid v \in V_{d}\right\}$ where $l_{v}+1$ is the scale of the $v$-links associated to $v$ (they are all at the same scale); 
- $J_{h}^{a}$ is the set of scales $j$ where the field $h$ is associated to a $f$-link: for each $j \in J_{h}^{a} h_{c}^{v}$ is external field for $y_{v}^{j}$. The same definition holds for $\bar{h}$;

- $j_{h}^{b}$ is the scale of the $h$-link associated to $h$. The same definition holds for $\bar{h}$;

- $C_{b}$ fixes the pairs $h-\bar{h}$ that form the $h$-links;

- $\varepsilon_{\mathcal{F}}$ is a sign coming from the horizontal forest formulas;

- $h L_{j}$ is the set of $h$-links of scale $j$ in $\mathcal{F}_{j}$. For each $h$-link $l$ we denote the corresponding field, antifield by $h_{l}, \bar{h}_{l}$. The vertices are denoted by $v(l)$ and $\bar{v}(l)$, their positions by $x_{l}\left(\bar{x}_{l}\right)$ and the cubes of the link containing them by $\Delta_{l}$ and $\bar{\Delta}_{l}$.

- $v L_{j}$ is the set of vertical links of scale $j$ associated to a vertex. We recall that each such vertex corresponds to a set of $v$-links in $\mathcal{F}_{j}$ connecting some subset $y$ at scale $j-1$ (which is already connected by $\mathcal{F}_{j-1}$ ) to its ancestor;

- $f L_{j}^{p}$ is the set of vertical links of scale $j$ associated to $p$ external fields. We recall that each such set of external fields corresponds to a set of $f$-links of scale $j$ and order $p(p=2,4,6)$ in $\mathcal{F}_{j}$ connecting some subset $y$ at scale $j-1$ (which is already connected by $\mathcal{F}_{j-1}$ ) to its ancestor;

- $w_{l}^{\prime}=w_{y_{k}^{j}}^{\prime}$ where $l$ is the $v$-links connecting $y_{k}^{j}$ to its ancestor. The same definition holds for $w_{l}^{\prime \prime}$;

- Defining

$\begin{cases}j_{m}(v)=\max \left\{j \mid y_{v}^{j} \text { connected to } \mathcal{A}\left(y_{v}^{j}\right) \text { by a } f-\operatorname{link}\right\} & \text { if } v \in \bar{V}_{d}, \\ j_{m}(v)=\max \left\{j<l_{v} \mid y_{v}^{j} \text { connected to } \mathcal{A}\left(y_{v}^{j}\right) \text { by a } f-\operatorname{link}\right\} & \text { if } v \in V_{d},\end{cases}$

(III.85)

we must have, for all $h_{c}^{v}, j_{c}^{v}>j_{m}(v)$. This bound can be understood as follows: a vertex $v$ cannot have $i_{v} \leq j_{m}(v)$. Indeed otherwise it would be internal for $y_{v}^{j_{m}(v)}$, and would have been chosen at that scale instead of the $f$-link connecting $y_{v}^{j_{m}(v)}$ to its ancestor. We remark that for $v \in V_{d}$ this argument only applies for scales $j<l_{v}$, since after $l_{v}$ the vertex can no longer be selected as a vertical connection. This explains the definition ([II.85). All these constraints are expressed in formula (III.84) by the function $v^{>j_{m}(v)}\left(j_{c}^{v}\right)$. 
Moreover, for each $v \in V_{d}$ we have inserted an additional sum

$$
\sum_{\rho_{v}}=\prod_{\left\{h_{c}^{v} \mid c \neq c_{v}\right\}} \sum_{\rho_{h}}
$$

where we recall that $c_{v}=\min \left\{c \in \sigma_{v}\right\}$ (匹1.60), and we define $\rho_{h}=1$ if $i_{v} \leq j_{h} \leq l_{v}$ and $\rho_{h}=2$ if $l_{v}<j_{h}$. Remark that for $c \in \sigma_{v}$ and $c \neq c_{v}$, or for $c=\alpha_{v}$, we must have $\rho_{h_{c}^{v}}=1$ by construction (Recall that $\alpha_{v}$ is defined in ([II.71)). On the other hand, if $h \in a$ we must have $\rho_{h}=2$ by construction.

- the values of $s_{j}$ depend on the $f$-links:

- $s_{j}(v, c)=1$ if $y_{v}^{j}$ is connected to its ancestor by a $v$-link or if $j \in J_{h_{c}^{v}}^{a}$ (which means $h_{c}^{v}$ is associated to a $f$-link connecting $y_{v}^{j}$ to its ancestor);

- $s_{j}(v, c)=w_{y_{v}^{j}}^{\prime \prime}$ if $y_{v}^{j}$ is connected to its ancestor by a $f$-link of order 6 and $j \notin J_{h_{c}^{v}}^{a}$;

- $s_{j}(v, c)=0$ if $y_{v}^{j}$ is connected to its ancestor by a $f$-link of order 2 or 4 and $j \notin J_{h_{c}^{v}}^{a}$.

- finally det $^{\prime}$ is the determinant remaining after the propagators corresponding to $h$-links have been extracted. The matrix element is

$$
M_{v c ; \bar{v} \bar{c}}^{\prime}\left(\left\{w_{l}\right\}\right)=\delta_{j_{c}^{v}, j \overline{\bar{c}}}\left[h_{\Delta_{v}^{j}, \Delta_{\bar{v}}^{j}}^{\mathcal{F}_{j}^{h}}(w) C_{\Delta_{v}^{j}, \Delta_{\bar{v}}^{j}}^{j}\left(x_{v}, x_{\bar{v}}\right)\right]_{j=j_{c}^{v}}
$$

where the weakening factor $h_{\Delta_{v}^{j}, \Delta_{\bar{v}}^{j}}^{\mathcal{F}_{j}^{h}}(w)$ is defined in (III.78), substituting in the formulae the general forest $\mathcal{F}$ with the horizontal forest $\mathcal{F}_{j}^{h}$.

Constrained attributions The non zero contributions are given by the following attributions:

- for $v \in V_{d}$ and $c \in \sigma_{v}$ we must have

$$
i_{v} \in I_{v}^{c}=\left[1+j_{m}(v), l_{v}\right]
$$

- for $v \in V_{d}$ and $c \notin \sigma_{v}$ we must have

$$
\begin{aligned}
& j_{c}^{v} \in J_{v}^{\prime c}=\left[i_{v}, l_{v}\right] \text { for } c=\alpha_{v} \\
& j_{c}^{v} \in J_{v}^{\prime c}=\left[j_{m}(v, c), j_{M}(v, c)\right] \quad c \neq \alpha_{v}
\end{aligned}
$$


where

$$
\begin{aligned}
& j_{m}(v, c)=1+i_{v} \quad \text { if } \quad h_{c}^{v} \notin a \text { and } \rho_{h_{c}^{v}}=1 \\
& j_{m}(v, c)=1+l_{v} \quad \text { if } \quad h_{c}^{v} \notin a \text { and } \rho_{h_{c}^{v}}=2 \\
& j_{m}(v, c)=1+\max \left\{j \in J_{h_{c}^{v}}^{a}\right\} \quad \text { if } \quad h_{c}^{v} \in a
\end{aligned}
$$

and $j_{M}(v, c)=l_{v}$ if $h_{c}^{v} \notin a$ and $\rho_{h_{c}^{v}}=1$, otherwise $j_{M}(v, c)=\min \left\{j>i_{v} \mid y_{v}^{j}\right.$ is connected to its ancestor by a $f$-link of order $p=2,4\}$, with the convention that $\min \emptyset=j_{M}+1$. Remark that $\alpha_{v}$ satisfies a special constraint because this is the field derived in order to extract a $v$-link at scale $l_{v}+1$, therefore it must satisfy $j_{\alpha_{v}}^{v} \leq l_{v}$;

- finally, for $v \in \bar{V}_{d}$ we must have

$$
j_{c}^{v} \in J_{v}^{c}=\left[j_{m}(v)+1, j_{M}+1\right]
$$

Reinserting attribution sums inside the determinant This is a key step for later bounds. We observe that for all $v \in \bar{V}_{d}$ the constraints $v^{j}$ and $v^{>j}$ on the attributions for each field hooked to $v$ are independent. Therefore we can reinsert all the sums inside the determinant (bringing with them the corresponding vertical weakening factors $w^{\prime}$ and $\left.w^{\prime \prime}\right)$.

On the other hand, for $v \in V_{d}$, the sum over attributions for $h_{c}^{v}$ with $c \notin \sigma_{v}$ are independent from each other but are all dependent from $i_{v}$. Therefore we can reinsert in the determinant the sums for $c \notin \sigma_{v}$ (with their vertical weakening factors), but we must keep the sum over $i_{v}$ outside the determinant. The weakening factors for all $c \neq c_{v}$ are inserted in the determinant. On the other hand for the particular field $h_{c_{v}}^{v}$ we keep outside the determinant the weakening factors $w^{\prime}$, as they will be used to perform certain sums, and reinsert the others in the determinant.

Therefore we can write the partition function as

$$
\begin{aligned}
& Z_{\Lambda}^{u}=\sum_{n=0}^{\infty} \frac{\lambda^{n}}{n !} \sum_{\Delta_{V}} \sum_{\mathcal{F}} \sum_{V_{d}, \alpha_{V_{d}}} \sum_{a, b, R} \sum_{l_{V_{d}}} \sum_{\left\{J_{h}^{a}\right\},\left\{J_{h}^{a}\right\}} \sum_{\left\{j_{h}^{b}\right\},\left\{j_{h}^{b}\right\}} \sum_{C_{b}} \varepsilon_{\mathcal{F}}\left[\prod_{v} \int_{\Delta_{v}} d^{4} x_{v}\right] \\
& {\left[\prod_{j=0}^{j_{M}+1}\left(\prod_{l \in h L_{j}} \int_{0}^{1} d w_{l}\right)\right]\left[\prod_{j=1}^{j_{M}+1}\left(\prod_{l \in v L_{j}} \int_{0}^{1} d w_{l}^{\prime}\right)\right]\left[\prod_{j=1}^{j_{M}+1}\left(\prod_{l \in f L_{j}^{6}} \int_{0}^{1} d w_{l}^{\prime \prime}\right)\right]} \\
& \left.\left.\left[\prod_{v \in V_{d}}\left(\sum_{n_{v} \sigma_{v} \rho_{v}} \sum_{i_{v} \in I_{v}^{c}}\right)\right]\right] \prod_{\substack{v \in V_{d} \\
c_{v} \neq \alpha_{v}}} \prod_{j=i_{v}}^{l_{v}} w_{y_{v}^{j}}^{\prime}\right]\left[\prod_{\substack{v \in V_{d} \\
c_{v}=\alpha_{v}}} \prod_{j=i_{v}}^{l_{v}-1} w_{y_{v}^{j}}^{\prime}\right]
\end{aligned}
$$




$$
\left[\prod_{j=0}^{j_{M}+1}\left(\prod_{l \in h L_{j}} C_{\Delta_{l} \bar{\Delta}_{l}}^{j}\left(x_{l}, \bar{x}_{l},\left\{w_{l^{\prime}}^{\prime}\right\},\left\{w_{l^{\prime}}^{\prime \prime}\right\}\right)\right)\right] \operatorname{det} M^{\prime \prime}\left(\left\{w_{l}\right\},\left\{w_{l}^{\prime}\right\},\left\{w_{l}^{\prime \prime}\right\}\right)
$$

where the matrix element is

$$
\begin{aligned}
& M_{v c ; \bar{v} \bar{c}}^{\prime \prime}\left(\left\{w_{l}\right\},\left\{w_{l}^{\prime}\right\},\left\{w_{l}^{\prime \prime}\right\}\right)=\left[\sum_{j_{c}^{v} \in \mathcal{I}_{c}^{v}} W_{v c}\left(j_{c}^{v}\right)\right] \\
& \delta_{j_{c}^{v}, j \overline{\bar{v}}}\left[h_{\Delta_{v}^{j}, \Delta_{\bar{v}}^{j}}^{\mathcal{F}_{j}^{h}}(w) C_{\Delta_{v}^{j}, \Delta_{\bar{v}}^{j}}^{j}\left(x_{v}, x_{\bar{v}}\right)\right]_{j=j_{c}^{v}}\left[\sum_{j_{c}^{v} \in \mathcal{I}_{\bar{c}}^{\bar{v}}} W_{\bar{v} \bar{c}}\left(j_{\bar{c}}^{\bar{v}}\right)\right]
\end{aligned}
$$

and the horizontal propagator is

$$
C_{\Delta_{l} \bar{\Delta}_{l}}^{j}\left(x_{l}, \bar{x}_{l},\left\{w_{l^{\prime}}^{\prime}\right\},\left\{w_{l^{\prime}}^{\prime \prime}\right\}\right)=W_{v_{l} c_{l}}(j) C_{\Delta_{l} \bar{\Delta}_{l}}^{j}\left(x_{l}, \bar{x}_{l}\right) W_{\bar{v}_{l} \bar{c}_{l}}(j)
$$

and $v_{l}, c_{l}$ and $\bar{v}_{l}, \bar{c}_{l}$ identify respectively the field and the antifield of the link. We defined

$$
\begin{aligned}
& \mathcal{I}_{c}^{v}=\left\{i_{v}\right\} \quad v \in V_{d}, c \in \sigma_{v} \\
& \mathcal{I}_{c}^{v}=J_{v}^{\prime c} \quad v \in V_{d}, c \notin \sigma_{v} \\
& \mathcal{I}_{c}^{v}=J_{v}^{c} \quad v \in \bar{V}_{d}
\end{aligned}
$$

and $I_{v}^{c}, J_{v}^{\prime c}$ and $J_{v}^{c}$ is the set of band attributions with the constraints due to the forest structure that we introduced above. Finally the definitions for the factors $W_{v c}$ are given below.

Vertical weakening factors The expression for $W_{v c}\left(j_{c}^{v}\right)$ is given by the $\Upsilon_{j}(v, c)$ and $s_{j}$ functions. Remark that

$$
\begin{array}{ll}
\Upsilon_{j}(v, c)=1 & \text { if } j<j_{c}^{v} \\
\Upsilon_{j}(v, c)=w_{y_{v}^{j}}^{\prime} & \text { if } j \geq j_{c}^{v}
\end{array}
$$

Actually we have to distinguish different cases.

If $v \in V_{d}, c=\alpha_{v}$ and $c \neq c_{v}$

$$
W_{v \alpha_{v}}\left(j_{\alpha_{v}}^{v}\right)=\left[\prod_{j=j_{\alpha_{v}}^{v}}^{l_{v}-1} w_{y_{v}^{j}}^{\prime}\right]\left[\prod_{j=i_{v}}^{j_{\alpha_{v}}^{v}-1} s_{j}\left(v, \alpha_{v}\right)\right] .
$$


If $v \in V_{d}, c \neq \alpha_{v}$ and $c \neq c_{v}$

$$
W_{v \alpha_{v}}\left(j_{c}^{v}\right)=\left[\prod_{j=j_{c}^{v}}^{l_{v}} w_{y_{v}^{j}}^{\prime}\right]\left[\prod_{j=i_{v}}^{j_{c}^{v}-1} s_{j}(v, c)\right]
$$

If $v \in V_{d}$ and $c=c_{v}$

$$
W_{v c_{v}}\left(j_{c_{v}}^{v}\right)=\left[\prod_{j=i_{v}}^{j_{c_{v}}^{v}-1} s_{j}\left(v, c_{v}\right)\right]
$$

Finally if $v \in \bar{V}_{d}$

$$
W_{v \alpha_{v}}\left(j_{c}^{v}\right)=\left[\prod_{j=j_{c}^{v}}^{j_{M}} w_{y_{v}^{j}}^{\prime}\right]\left[\prod_{j=i_{v}}^{j_{c}^{v}-1} s_{j}(v, c)\right]
$$

where we take the convention that a void product is 1 . Therefore for $v \in V_{d}$ and $\rho_{h}=1$ the product over $s_{j}$ is reduced to 1 and for $v \in V_{d}$ and $\rho_{h}=2$ the product over $w^{\prime}$ is reduced to 1 .

\section{III.5 Connected components}

Now, at each order $n$ we can factorize the connected components, namely the polymers. The forest $\mathcal{F}$ is connected if at the highest slice index (hence the lowest energy scale) there is only one connected component. Remark that $\mathcal{F}$ could have no link for any $j>j_{\mathcal{F}}$. In this case the forest is connected if $\mathcal{F}_{j_{\mathcal{F}}}$ has only one connected component.

The partition function is written as

$$
Z_{\Lambda}^{u}=\sum_{k_{Y}=0}^{\infty} \frac{1}{k_{Y} !} \sum_{\substack{Y_{1}, \ldots, Y_{k_{Y}} \\ u_{q} Y_{q}=\mathcal{D}, Y_{q} \cap Y_{q^{\prime}}=\emptyset}} \prod_{q} A\left(Y_{q}\right)
$$

where $k_{Y}$ is the number of different connected polymers $Y_{q}$ and the amplitude for a polymer $Y$ is defined as

$$
A(Y)=\sum_{n=0}^{\infty} \frac{\lambda^{n}}{n !} \sum_{\Delta_{V}} \sum_{\mathcal{F}_{M_{Y}}^{c}} \sum_{V_{d}, \alpha_{V_{d}}} \sum_{a, b, R} \sum_{l_{V_{d}}} \sum_{\left\{J_{h}^{a}\right\},\left\{J_{\bar{h}}^{a}\right\}} \sum_{\left\{j_{h}^{b}\right\},\left\{j_{\bar{h}}^{b}\right\}} \sum_{C_{b}} \varepsilon_{\mathcal{F}}\left[\prod_{v} \int_{\Delta_{v}} d^{4} x_{v}\right]
$$




$$
\begin{aligned}
& {\left[\prod_{j=m_{Y}}^{M_{Y}}\left(\prod_{l \in h L_{j}} \int_{0}^{1} d w_{l}\right)\right]\left[\prod_{j=m_{Y}+1}^{M_{Y}}\left(\prod_{l \in v L_{j}} \int_{0}^{1} d w_{l}^{\prime}\right)\right]\left[\prod_{j=m_{Y}+1}^{M_{Y}}\left(\prod_{l \in f L_{j}^{6}} \int_{0}^{1} d w_{l}^{\prime \prime}\right)\right]} \\
& {\left[\prod_{v \in V_{d}}\left(\sum_{n_{v} \sigma_{v} \rho_{v}} \sum_{i_{v} \in I_{v}^{c}}\right)\right]\left[\prod_{\substack{v \in V_{d} \\
c_{v} \neq \alpha_{v}}} \prod_{j=i_{v}}^{l_{v}} w_{y_{v}^{j}}^{\prime}\right]\left[\prod_{\substack{v \in V_{d} \\
c_{v}=\alpha_{v}}} \prod_{j=i_{v}}^{l_{v}-1} w_{y_{v}^{j}}^{\prime}\right]} \\
& {\left[\prod_{j=m_{Y}}^{M_{Y}}\left(\prod_{l \in h L_{j}} C_{\Delta_{l} \bar{\Delta}_{l}}^{j}\left(x_{l}, \bar{x}_{l},\left\{w_{l^{\prime}}^{\prime}\right\},\left\{w_{l^{\prime}}^{\prime \prime}\right\}\right)\right)\right] \operatorname{det} M^{\prime \prime}\left(\left\{w_{l}\right\},\left\{w_{l}^{\prime}\right\},\left\{w_{l}^{\prime \prime}\right\}\right)}
\end{aligned}
$$

where $\mathcal{F}_{M_{Y}}^{c}$ is any connected forest over $Y$. The spatial integral for each $v$ is still written in terms of cubes in $\mathcal{D}_{0}$, but all sums are restricted to the polymer. This means that $\Delta \in \mathcal{D}_{j}$ becomes $\Delta \in \mathcal{D}_{j} \cap Y$ and so on. Remark that $\mathcal{F}_{M_{Y}}^{c}$ has no link at scale $j<m_{Y}$.

\section{III.6 Main result}

Now we have nearly succeeded in computing the logarithm of $\mathcal{Z}$. Actually (IIII.101) would be the exponential of $A(Y)$, if there was no constraint $Y_{q} \cap$ $Y_{q^{\prime}}=\emptyset, \cup_{q} Y_{q}=\mathcal{D}$. Taking out these conditions and computing the logarithm is the purpose of the so called Mayer expansion [R].

By translation invariance, a Mayer expansion converges essentially if the following condition holds:

$$
\sum_{\substack{Y \\ 0 \in Y}}|A(Y)| e^{|Y|} \leq 1
$$

(where $|Y|$ is the cardinal of $Y$, hence the total number of cubes of all scales forming $Y$ ). If we perform power counting, we find that all sub-polymers of $Y, Y_{k}^{j}$, with $\left|E\left(Y_{k}^{j}\right)\right|=2,4$ need renormalization. This is postponed to a future publication f. To start with a simpler situation, in this paper we

\footnotetext{
${ }^{5}$ The constraint that $Y$ must be connected implies that the term at order $n$ is zero unless $n$ is big enough (in order to be able to connect $Y$ ).

${ }^{6}$ In this future publication, we plan in fact to renormalize only the 2-point function, and to bound the logarithmic divergence of the 4-point functions by the condition $\lambda|\log T| \leq$ $K$, like in [DR2].
} 
restrict ourselves to the case $\left|E\left(Y_{k}^{j}\right)\right|>4$ for all $j<j_{M}+1$. We call this subset the convergent attributions for $Y$ and we denote the corresponding amplitudes by $A_{c}(Y)$. Remark that $A_{c}(Y)$ contains only $f$-links of order 6 . We therefore prove the following theorem, which is a $3-\mathrm{d}$ analog of [FMRT] and [DR1].

Theorem For any $L>0$, there exists $K>0$, such that if

$$
|\lambda \ln T| \leq K
$$

we have

$$
\sum_{\substack{Y \\ 0 \in Y}}\left|A_{c}(Y)\right| L^{|Y|} \leq 1
$$

The sum is performed over all polymers that contain the position $x=0$, and $A_{c}(Y)$ is the amplitude of $Y$ restricted to the convergent attributions.

The rest of the paper is devoted to the proof of this theorem, and from now on we further assume $K \leq 1$.

\section{Proof}

The general idea is to bound the determinant by a Hadamard inequality, and to sum over the horizontal structures using the horizontal propagators decay. The Hadamard inequality generally costs a factor

$$
n^{n}|\ln T|^{\left|\bar{V}_{d} \backslash V_{b}\right|+(1-\varepsilon)\left|V_{d} \cup V_{b}\right|}
$$

where $0<\varepsilon<1$ and $V_{b}$ is the set of vertices hooked to some horizontal link:

$$
V_{b}=\left\{v \in V \mid h_{c}^{v} \in b \text { for some } c\right\} .
$$

The factor $n^{n}$ is bounded by the global $1 / n$ ! symmetry factor of the vertices, up to a factor $e^{n}$ by Stirling formula, which is absorbed in the constant $K^{\prime}$ (see however the remark in the Introduction). The logarithm is bounded by a fraction of the small coupling constant $\lambda^{n}$. A delicate point is to prove that the factor $\varepsilon$ is strictly positive $\varepsilon>0$, since we need to spare a fraction of $\lambda$ at each derived vertex $v \in V_{d} \cup V_{b}$ in order to extract a small factor per cube. This factor is necessary to bound the last sum over the polymer size and shape. 
In the following we will denote fields only by $h$ (not $h_{c}^{v}$ ) and antifields by $\bar{h}$. The corresponding vertex is $v_{h}, v_{\bar{h}}$, the field index is $c_{h} \in C$, the antifield index $c_{\bar{h}} \in \bar{C}(C$ and $\bar{C}$ are introduced in section II.5), their slice indices are $j_{h}, j_{\bar{h}}$ and their vertex position is $x_{h}, x_{\bar{h}}$.

In order to bound the amplitude of a polymer $A(Y)$ we must introduce the auxiliary slice decoupling of section II.4. For each propagator extracted from the determinant we write

$$
\begin{aligned}
C_{\Delta_{l} \bar{\Delta}_{l}}^{j}\left(x_{l}, \bar{x}_{l},\left\{w_{l^{\prime}}^{\prime}\right\},\left\{w_{l^{\prime}}^{\prime \prime}\right\}\right) & =\sum_{k=0}^{k_{M}(j)} C_{\Delta_{l} \bar{\Delta}_{l}}^{j k}\left(x_{l}, \bar{x}_{l},\left\{w_{l^{\prime}}^{\prime}\right\},\left\{w_{l^{\prime}}^{\prime \prime}\right\}\right) \quad(\mathrm{IV} .108) \\
& =\sum_{k_{h_{l}} k_{\bar{h}_{l}}} \delta_{k_{h_{l}}, k_{\bar{h}_{l}}} C_{\Delta_{l} \bar{\Delta}_{l}}^{j k_{h_{l}}}\left(x_{l}, \bar{x}_{l},\left\{w_{l^{\prime}}^{\prime}\right\},\left\{w_{l^{\prime}}^{\prime \prime}\right\}\right)
\end{aligned}
$$

where

$$
C_{\Delta_{l} \bar{\Delta}_{l}}^{j k}\left(x_{l}, \bar{x}_{l},\left\{w_{l^{\prime}}^{\prime}\right\},\left\{w_{l^{\prime}}^{\prime \prime}\right\}\right)=W_{v_{l} c_{l}}(j) C_{\Delta_{l} \bar{\Delta}_{l}}^{j k}\left(x_{l}, \bar{x}_{l}\right) W_{\bar{v}_{l} \bar{c}_{l}}(j)
$$

$C^{j k}$ is defined in (II.35) and $W_{h}(j)$ corresponds to the function $W_{v c}(j)$ defined in (III.97-[II.100). The matrix element is written as

$$
\begin{gathered}
M_{h ; \bar{h}}^{\prime \prime}\left(\left\{w_{l}\right\},\left\{w_{l}^{\prime}\right\},\left\{w_{l}^{\prime \prime}\right\}\right)=\sum_{k_{h} k_{\bar{h}}} \sum_{j \in \mathcal{I}_{h} \cap \mathcal{I}_{\bar{h}} \cap J\left(k_{h}\right)} \delta_{k_{h}, k_{\bar{h}}} \\
{\left[W_{h}(j)\right]\left[h_{\Delta_{h}^{j}, \Delta_{\bar{h}}^{j}}^{\mathcal{F}_{j}^{h}}(w)\right] C_{\Delta_{h}^{j}, \Delta_{\bar{h}}^{j}}^{j k_{h}}\left(x_{h}, x_{\bar{h}}\right)\left[W_{\bar{h}}(j)\right]}
\end{gathered}
$$

where we have exchanged the sums over $j_{h}$ and $k_{h}, J(k)$ is defined in (II.49) and the interval $\mathcal{I}_{h}$ corresponds to the interval $\mathcal{I}_{c}^{v}$ defined in (III.95). Finally we denote by $\Delta_{h}^{j}$ the cube $\Delta_{v_{h}}^{j}$. The same definitions hold for $\bar{h}$. The sums over $k_{h}$ and $k_{\bar{h}}$ are extracted from the determinant by multilinearity. We need now to reorganize the sum over $Y$ according to a tree structure analogous to the "Gallavotti-Nicoló tree" [GN]. that is called here $S$.

\section{IV.1 The $S$ structure}

Let $M_{Y}$ be the lowest scale of the polymer. $S$ is a rooted tree that pictures the inclusion relations for the connected components of $Y$ at each scale and the type of vertical connection (vertex or field). In this rooted tree the extremal leaves are pictured as dots and the other vertices as circles. A circle at layer 


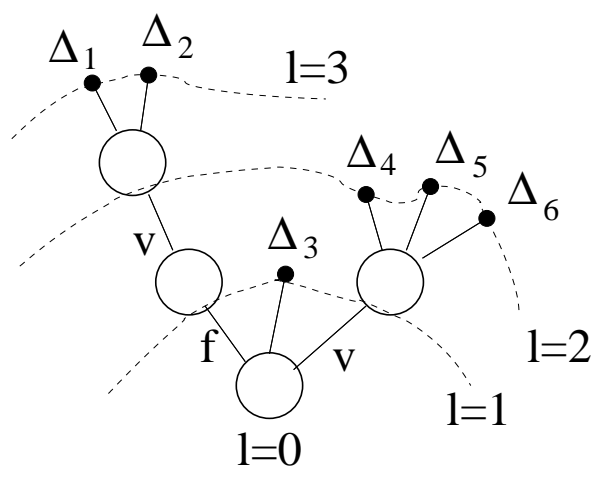

Figure 7: Example of $S$

$l$ represents a connected subpolymer at scale $j=M_{Y}-l$. A leaf at layer $l$ by convention represents an extremal summit cube, that is a cube such that $\operatorname{Ex}(\Delta)=\Delta$ (no cube above), whose scale is $M_{Y}-l+1$. The highest layer fixes the scale $m_{Y}: l_{\max }=M_{Y}-m_{Y}+1$ (as at scale $M_{Y}-l_{\max }$ there are only leaves, hence no cubes) and satisfies $l_{\max }-1 \leq j_{M}$.

There are two types of links in $S$ : the leaf-links which join a leaf to a circle, and the circle-links which join two circles. To each circle-link corresponds a vertical block in the multiscale expansion, and we can associate to it a label $f$ or $v$ depending if this block is associated to a vertex or to external fields $t$.

An example of $S$ structure is given in Fig.7 and two possible polymers corresponding to this structure are given in Fig.8 a and b. We remark that $S$ fixes in a unique way the number and scales of the extremal summit cubes, but that several polymers, with different total number of cubes, may correspond to the same structure $S$.

In order to fix this total number of cubes, we introduce for each circle-link of $S$ a further number which fixes the number of vertical links (wich are $v$ links or $f$-links depending of the type of the circle-link) selected in the block in section III.3. Since there is one vertical link per ancestor cube, this number is the number of ancestor cubes of the connected component $y$ corresponding to the circle at the top of the circle-link. We call this collection of indices $V L$. $S$ and $V L$ together fix the number $|Y|$ of cubes in $Y$. For instance the situations in Figga and b. correspond to the same $S$, shown in Fig.9 d. But

${ }^{7}$ We remark that the circles at level $l$ connected only to leaves at level $l+1$ must be connected to the previous circle at level $l-1$ by a $v$-circle-link. Indeed each of the extremal summit cubes forming that circle must contain at least one vertex. 


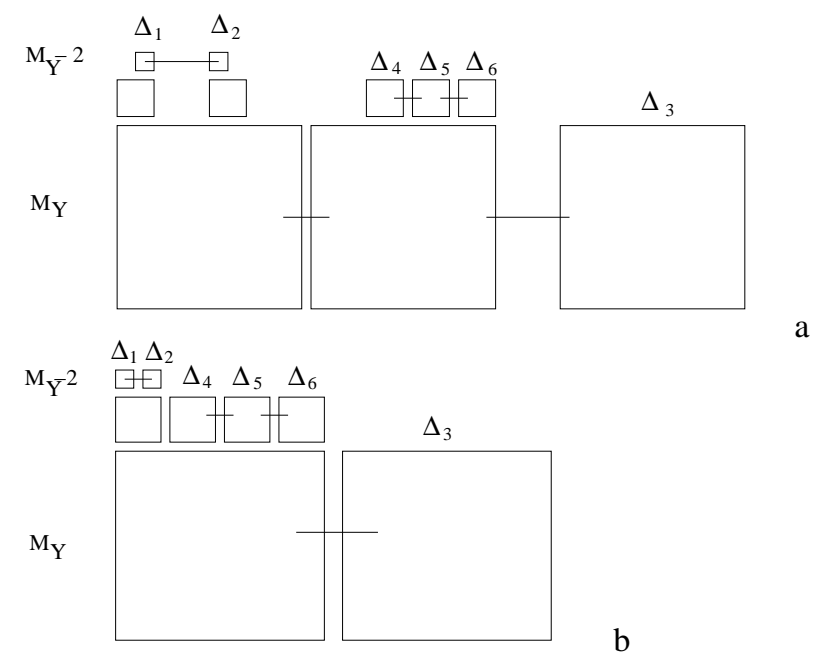

Figure 8: Two possible polymers corresponding to $S$

the case a) corresponds to an index $V L=\{1\}$ for the unique circle-link and to 4 cubes in $Y$, whether the case b) corresponds to an index $V L=\{2\}$ and to 5 cubes in $Y$,

Finally when $S$ and $V L$ are given, we can label all the cubes of $Y$, and we fix the subset $B_{S}$ of those cubes of $Y$ which are summit cubes. They are those with non-zero exposed volume: $|E x(\Delta)|>0^{8}$. Nevertheless we remark that there is still some ambiguity, as even $V L$ and $B_{S}$ cannot distinguish between Fig.9 $\mathbf{b}$ and $\mathbf{c}$, and the position of the cubes of $Y$ is not yet fixed.

\section{IV.2 The reorganized sum}

The sum (III.105) is then reorganized in terms of the structure $S$ as

$$
\begin{gathered}
\sum_{\substack{Y \\
0 \in Y}}\left|A_{c}(Y)\right| L^{|Y|} \leq \sum_{M_{Y}} \sum_{S} \sum_{V L} L^{|Y|} \sum_{B_{S}} \sum_{\left\{x_{\Delta}\right\}^{c}} \mid \sum_{n=0}^{\infty} \frac{\lambda^{n}}{n !} \sum_{V_{d}, \alpha_{V_{d}}} \sum_{a, b, R} \sum_{\left\{v_{l}\right\}_{l \in v L}} \\
\sum_{n_{V_{d}} \sigma_{V_{d}} \rho_{V_{d}}} \sum_{\left\{n_{\Delta}\right\}_{\Delta \in B_{S}}} \sum_{\Delta_{\bar{V}_{d}}^{c}}\left[\prod_{v \in V_{d}} \sum_{i_{v} \in I_{v}^{c}} \sum_{\Delta_{v} \in \mathcal{D}_{i_{v}} \cap Y}\right] \sum_{\left\{J_{h}^{a}\right\},\left\{J_{h}^{a}\right\}} \sum_{\left\{j_{h}^{b}\right\},\left\{j j_{\bar{h}}^{b}\right\}} \sum_{\left\{k_{h}\right\},\left\{k_{\bar{h}}\right\}}
\end{gathered}
$$

\footnotetext{
${ }^{8}$ Actually $B_{S}$ only really fixes the non-extremal summit cubes $\Delta$ (with $0<|E x(\Delta)|<$ $|\Delta|$ ) since the extremal summit cubes with $\operatorname{Ex}(\Delta)=\Delta$ were already known from the data in $S$.
} 

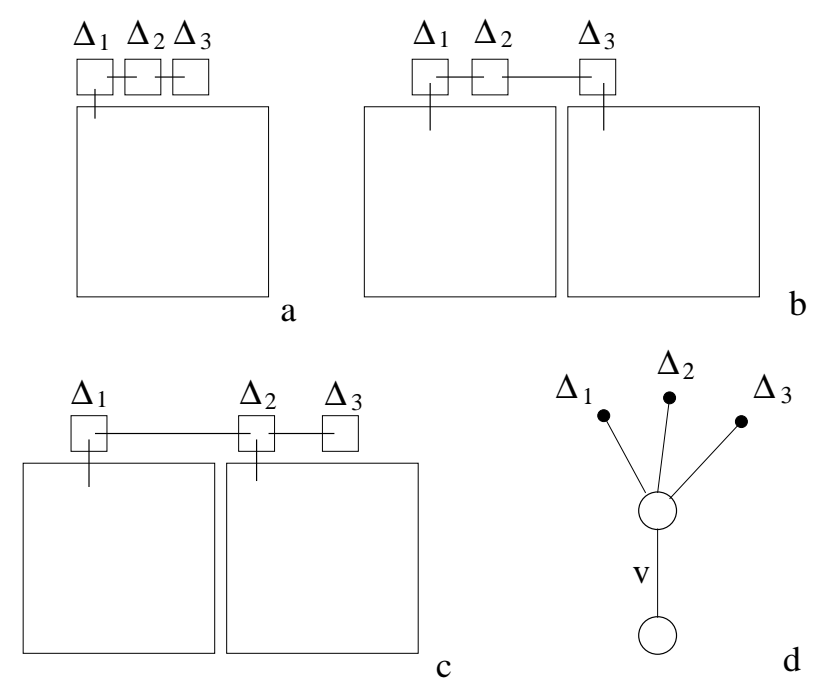

Figure 9: a,b,c: three polymers corresponding to the same $S$ shown in d: $V L$ can distinguish a from $b$, but not $b$ from $c$

$$
\begin{aligned}
& {\left[\prod_{j=m_{Y}}^{M_{Y}} \prod_{k=1}^{c_{j}} \sum_{T_{j k}}\right]\left[\prod_{v \in \bar{V}_{d}} \int_{E x\left(\Delta_{v}\right)} d x_{v}\right]\left[\prod_{v \in V_{d}} \int_{\Delta_{v}} d x_{v}\right] \varepsilon_{\mathcal{F}}} \\
& {\left[\prod_{j=m_{Y}}^{M_{Y}}\left(\prod_{l \in h L_{j}} \int_{0}^{1} d w_{l}\right)\right]\left[\prod_{j=m_{Y}+1}^{M_{Y}}\left(\prod_{l \in v L_{j}} \int_{0}^{1} d w_{l}^{\prime}\right)\right]\left[\prod_{j=m_{Y}+1}^{M_{Y}}\left(\prod_{l \in f L_{j}^{6}} \int_{0}^{1} d w_{l}^{\prime \prime}\right)\right]} \\
& {\left[\prod_{j=m_{Y}}^{M_{Y}} \prod_{k=1}^{c_{j}}\left[\prod_{l \in T_{j k}} C_{\Delta_{l} \bar{\Delta}_{l}}^{j k_{h_{l}}}\left(x_{l}, \bar{x}_{l},\left\{w_{l^{\prime}}^{\prime}\right\},\left\{w_{l^{\prime}}^{\prime \prime}\right\}\right)\right] \delta_{k_{h_{l}} k_{\bar{h}_{l}}}\right]} \\
& {\left[\operatorname{det} M^{\prime \prime}\left(\left\{w_{l}\right\},\left\{w_{l}^{\prime}\right\},\left\{w_{l}^{\prime \prime}\right\},\left\{k_{h, \bar{h}}\right\}\right)\right]\left[\prod_{\substack{v \in V_{d} \\
c v \neq \alpha_{v}}} \prod_{j=i_{v}}^{l_{v}} w_{y_{v}^{j}}^{\prime}\right]\left[\prod_{\substack{v \in V_{d} \\
c_{v}=\alpha_{v}}}^{l_{j=i_{v}}-1} \prod_{y_{v}^{j}}^{\prime} w^{\prime}\right] \mid}
\end{aligned}
$$

where

- $\left\{x_{\Delta}\right\}^{c}$ chooses the position of each cube in the polymer, constrained by $S, V L$ and $B_{S}$, with the additional constraint that at the lowest level $M_{Y}$ there is one cube containing the origin $x=0$.

- $v_{l}$ is the vertex $v \in V_{d}$ associated to the vertical link $l \in v L$ where $v L=\cup_{j} v L_{j}$. Remark that once we know $v_{l}$ for each $l \in v L$, we 
automatically know $l_{v}$ for all $v \in V_{d}$. The vertices of $V_{d}$ are from now on said to be localized in the cube $\Delta_{i_{v}} \in \mathcal{D}_{i_{v}}$ to which they belong.

- $n_{B_{S}}=\left\{n_{\Delta}\right\}_{\Delta \in B_{S}}$ gives the number of vertices in $\bar{V}_{d}$ localized in each summit cube (recall ([II.65): $n_{B_{S}}=\left\{n_{\Delta} \mid \Delta \in B_{S}\right\}$ with the constraint $\sum_{\Delta \in B_{S}} n_{\Delta}=\left|\bar{V}_{d}\right|=n-\left|V_{d}\right|$.

- $n_{V_{d}}, \sigma_{V_{d}}, \rho_{V_{d}}$ are the assignments $n_{v}, \sigma_{v}, \rho_{v} \forall v \in V_{d}$.

- $\Delta_{\bar{V}_{d}}^{c}$ chooses which vertices $v \in \bar{V}_{d}$ are localized in each summit cube: $\Delta_{\bar{V}_{d}}^{c}=\left\{\Delta_{v}\right\}_{v \in \bar{V}_{d}}$ with the constraint $\#\left\{v \mid v \in \bar{V}_{d}, \Delta_{v}=\Delta\right\}=n_{\Delta}$, $\forall \Delta \in B_{S}$. The spatial integral for each $v \in \bar{V}_{d}$ is then performed over the exposed volume of the corresponding cube $\operatorname{Ex}\left(\Delta_{v}\right)$ (see (III.65)).

- $k_{h}$ fixes the value of an auxiliary scale (defined in section II.4) that will be used in the propagator analysis; $k_{\bar{h}}$ is the same thing for antifields.

- $T_{j k}$ chooses the tree connecting the generalized cubes $\tilde{\Delta} \in y_{j}^{k}$ by $h$-links of scale $j$. To fix $T_{j k}$ one has to choose the $h$-links and the corresponding fields. As the fields (antifields) that must contract at scale $j$ in order to create $T_{j k}$ are already fixed by $b, j_{h}^{b}$ and $j_{h}^{b}$, we only have to fix the field-antifield pairing $C_{b}$ restricted to $y_{j}^{k}$.

\section{IV.3 Bounding the determinant}

In order to bound the main determinant we apply the following

Hadamard inequalities If $M$ is a $n \times n$ matrix with elements $M_{i j}$, its determinant satisfies the following bounds

$$
\begin{array}{ll}
\text { Hr : } & |\operatorname{det} M| \leq \prod_{i=1}^{n}\left[\sum_{j=1}^{n}\left|M_{i j}\right|^{2}\right]^{\frac{1}{2}} \\
\text { Hc : } & |\operatorname{det} M| \leq \prod_{j=1}^{n}\left[\sum_{i=1}^{n}\left|M_{i j}\right|^{2}\right]^{\frac{1}{2}}
\end{array}
$$

where $\mathrm{Hr}$ is obtained by considering each row as a n-component vector, and $H c$ by considering each column as a n-component vector.

We remark that these two inequalities are both true, but not identical. In our case it is crucial to optimize as much as possible our bounds, and to 
use either the row or the column inequality depending of the kind of fields involved and of various scaling and occupation factors.

Before expanding the determinant in ([V.111) we distinguish therefore five different types of fields (antifields) denoted by an index $\alpha_{h}, \alpha_{\bar{h}}$ :

$$
\begin{array}{lll}
\alpha_{h}=1 & \text { if } & v_{h} \notin V_{d} \\
\alpha_{h}=2 & \text { if } & v_{h} \in V_{d}, c_{h} \neq c_{v} \text { and } \rho_{h}=1 \\
\alpha_{h}=3 & \text { if } & v_{h} \in V_{d}, c_{h} \neq c_{v}, h \notin a \text { and } \rho_{h}=2 \\
\alpha_{h}=4 & \text { if } & v_{h} \in V_{d}, h \in a \\
\alpha_{h}=5 & \text { if } & v_{h} \in V_{d} \text { and } c_{h}=c_{v}
\end{array}
$$

The same definitions hold for antifields $\bar{h}$. The case $\alpha_{h}=1$ is the most general one. This is a partition, since neither the fields with $\rho_{h}=1$ and $c_{h} \neq c_{v}$ nor the special fields $h$ with $v_{h} \in V_{d}$ and $c_{h}=c_{v}$ can belong to $a$.

We now define for each field $h$ a weight $I_{h}$ which depends of the type of the field as follows:

$$
\begin{array}{ll}
\alpha_{h}=1: & I_{h}=n_{\Delta_{h}} M^{-4 i_{\Delta_{h}}} f_{\Delta_{h}}^{-1} \\
\alpha_{h}=2: & I_{h}=M^{-4 i_{v_{h}}} \\
\alpha_{h}=3: & I_{h}=M^{-4 l_{v_{h}}} \\
\alpha_{h}=4: & I_{h}= \\
\alpha_{h}=5: & I_{h}=M^{-4 i_{h}}
\end{array}
$$

where $\Delta_{h}$ is the cube where the vertex $v_{h}$ is localized. For $\Delta \in B_{S}$ we defined $f_{\Delta}$ as the exposed fraction of the volume $|\Delta|=M^{4 i_{\Delta}}$, and $n_{\Delta}$ as the number of vertices in $\bar{V}_{d}$ localized in the summit cube $\Delta$. Finally, for each $h \in a$ the scale $i_{h}$ is defined as

$$
i_{h}=\max J_{h}^{a}
$$

We remark that actually $h \in a$ can only have attributions $j \geq 1+i_{h}$. The same definitions hold for $\bar{h}$.

The Hadamard inequality will be either of the row or of the column type depending on whether the ratio of weights of the fields involved is larger or smaller than 1 . In fact we need to discretize these ratios in order to transfer some factors from fields to antifields and conversely and to obtain a correct bound. To implement this program we introduce an auxiliary expansion called the weight expansion. 


\section{IV.3.1 The weight expansion}

We expand

$$
h=\sum_{\beta_{h}=1}^{5} h^{\beta_{h}}
$$

where $h^{\beta_{h}}$ means that $h$ can contract only with $\bar{h}$ such that $\alpha_{\bar{h}}=\beta_{h}$. The same holds for the antifields.

Finally, we expand each $h^{\beta_{h}}\left(\bar{h}^{\beta_{\bar{h}}}\right)$ as

$$
h^{\beta_{h}}=\sum_{r \in \mathbb{Z}} h^{\beta_{h}}(r)
$$

where $h^{\beta_{h}}(r)$ means that $h$ can contract only with $\bar{h}$ such that

$$
\left.\left.\frac{I_{h}}{I_{\bar{h}}} \in I_{r} ; \quad I_{0}=[1], I_{r}=\right] 2^{r-1}, 2^{r}\right] \text { if } r>0, \quad I_{r}=\left[2^{r}, 2^{r+1}[\text { if } r<0\right.
$$

We remark that the intervals $I_{r}$ are disjoint with $\left.\cup_{r \in \mathbb{Z}} I_{r}=\right] 0,+\infty[$ and that with this definition $h(r)$ can contract only with antifields $\bar{h}\left(r^{\prime}\right)$ with $r^{\prime}=-r$. The same holds for the antifields.

The special fields or antifields of type 5 require an additional expansion. We define for each such field $h$ an occupation number $n(h)$ which is the number of derived vertices localized in the same cube than $h$

$$
n(h)=n_{d}\left(\Delta_{i_{h}}\right)=\mid\left\{\text { vertices in } V_{d} \text { localized in the cube } \Delta_{i_{h}}\right\} \mid
$$

We remark that $n_{d}\left(\Delta_{i_{h}}\right)$ has nothing to do with $n_{\Delta_{v_{h}}}$ in general, since these numbers concern respectively $V_{d}$ and $\bar{V}_{d}$. We recall that the vertices $v \in V_{d}$ are localized in the cube of $\mathcal{D}_{i_{v}}$ to which they belong, whether the vertices of $\bar{V}_{d}$ are localized in the summit cube to which they belong.

By convention, for any field not of type 5 we put

$$
n(h)=1
$$

The same definitions hold for the antifields. Now we expand each field as

$$
h^{\beta}(r)=\sum_{s \in \mathbb{Z}} h^{\beta}(r, s)
$$


where $h^{\beta_{h}}(r, s)$ means that $h$ can contract only with $\bar{h}$ such that

$$
\frac{n(h)}{n(\bar{h})} \in I_{s}
$$

where $I_{s}$ is defined like $I_{r}$ in ([V.119). We remark that this additional $s$ expansion is trivial (reduced to the term $s=0$ ) unless $\alpha$ or $\beta$ equals 5 , and that for $\alpha \neq 5 \beta=5, s$ is negative: $s \leq 0$. Symmetrically for $\alpha=5 \beta \neq 5, s$ is positive: $s \geq 0$.

Summarizing all constraints, the field $h^{\beta_{h}}(r, s)$ contracts only with antifields $\bar{h}^{\beta_{\bar{h}}}\left(r^{\prime}, s^{\prime}\right)$ such that $\beta_{h}=\alpha_{\bar{h}}, \beta_{\bar{h}}=\alpha_{h}, k_{h}=k_{\bar{h}}, r^{\prime}=-r$ and $s^{\prime}=-s$. Therefore we have

$$
\left|\left\{h^{\beta}(r, s) \mid \alpha_{h}=\alpha, k_{h}=k\right\}\right|=\left|\left\{\bar{h}^{\alpha}(-r,-s) \mid \alpha_{\bar{h}}=\beta, k_{\bar{h}}=k\right\}\right| .
$$

The determinant in (IV.111) is now written as

$$
\operatorname{det} M^{\prime \prime}=\sum_{\left\{\beta_{h}\right\}\left\{\beta_{\bar{h}}\right\}} \sum_{\left\{r_{h}\right\},\left\{r_{\bar{h}}\right\}} \sum_{\left\{s_{h}\right\},\left\{s_{\bar{h}}\right\}}\left[\prod_{r, s \in \mathbb{Z}} \operatorname{det} \mathcal{M}_{r, s}\left(\left\{\beta_{h}\right\}\left\{\beta_{\bar{h}}\right\}\right)\right]
$$

where the sums over $r_{h}, r_{\bar{h}}, s_{h}, s_{\bar{h}}, \beta_{h}$ and $\beta_{\bar{h}}$ are extracted from the determinant by multilinearity, and $\mathcal{M}_{r, s}$ is the matrix containing only fields with $r_{h}=r$ (therefore only antifields with $r_{\bar{h}}=-r$ ) and $s_{h}=s$ (therefore only antifields with $s_{\bar{h}}=-s$ ) We take the convention that $\mathcal{M}_{r, s}=1$ if there is no field with $r_{h}=r$ and $s_{h}=s$. We recall that the sums over $s_{h}$ and $s_{\bar{h}}$ are restricted by some constraints: $s=0$ unless $\beta_{h}$ or $\beta_{\bar{h}}$ equals $5, s \leq 0$ for $\beta_{h}=5, \beta_{\bar{h}} \neq 5$, and $s \geq 0$ for $\beta_{h} \neq 5, \beta_{\bar{h}}=5$.

Now we can insert absolute values inside the sums and (IV.111) can be bounded by

$$
\begin{gathered}
\sum_{\substack{Y \\
0 \in Y}}\left|A_{c}(Y)\right| L^{|Y|} \leq \sum_{M_{Y}} \sum_{S} \sum_{V L} L^{|Y|} \sum_{B_{S}} \sum_{\left\{x_{\Delta}\right\}^{c}} \sum_{n=0}^{\infty} \frac{|\lambda|^{n}}{n !} \sum_{V_{d}, \alpha_{V_{d}}} \sum_{a, b, R} \sum_{\left\{v_{l}\right\}_{l \in v L}} \\
\sum_{n_{V_{d} \sigma_{V_{d}} \rho_{V_{d}}}} \sum_{\left\{n_{\Delta}\right\}} \sum_{\Delta \in B_{S}}\left[\prod_{\Delta_{\bar{V}_{d}}^{c}} \sum_{v \in V_{d}} \sum_{i_{v} \in I_{v}^{c}}\right] \sum_{\Delta_{v} \in \mathcal{D}_{i_{v}} \cap Y} \sum_{\left\{J_{h}^{a}\right\},\left\{J_{\bar{h}}^{a}\right\}} \sum_{\left\{j_{h}^{b}\right\},\{j \bar{h}\}} \\
{\left[\prod_{j=m_{Y}} \prod_{k=1}^{M_{Y}} \sum_{\left.T_{j k}\right\},\left\{k_{\bar{h}}\right\}}\right]\left[\prod_{v \in \bar{V}_{d}} \int_{E x\left(\Delta_{v}\right)} d x_{v}\right]\left[\prod_{v \in V_{d}} \int_{\Delta_{v}} d x_{v}\right]}
\end{gathered}
$$




$$
\begin{aligned}
& {\left[\prod_{j=m_{Y}}^{M_{Y}}\left(\prod_{l \in h L_{j}} \int_{0}^{1} d w_{l}\right)\right]\left[\prod_{j=m_{Y}+1}^{M_{Y}}\left(\prod_{l \in v L_{j}} \int_{0}^{1} d w_{l}^{\prime}\right)\right]\left[\prod_{j=m_{Y}+1}^{M_{Y}}\left(\prod_{l \in f L_{j}^{6}} \int_{0}^{1} d w_{l}^{\prime \prime}\right)\right]} \\
& {\left[\prod_{j=m_{Y}}^{M_{Y}} \prod_{k=1}^{c_{j}}\left[\prod_{l \in T_{j k}}\left|C_{\Delta_{l} \bar{\Delta}_{l}}^{j k_{l}}\left(x_{l}, \bar{x}_{l},\left\{w_{l^{\prime}}^{\prime}\right\},\left\{w_{l^{\prime}}^{\prime \prime}\right\}\right)\right|\right] \delta_{k_{h_{l}} k_{\bar{h}}}\right] \sum_{\left\{\beta_{h}\right\}\left\{\beta_{\bar{h}}\right\}} \sum_{\left\{r_{h}\right\},\left\{r_{\bar{h}}\right\}}} \\
& \sum_{\left\{s_{h}\right\},\left\{s_{\bar{h}}\right\}}\left[\prod_{r, s \in \mathbb{Z}}\left|\operatorname{det} \mathcal{M}_{r, s}\left(\left\{\beta_{h}\right\}\left\{\beta_{\bar{h}}\right\}\right)\right|\right]\left[\prod_{\substack{v \in V_{d} \\
c_{v} \neq \alpha_{v}}} \prod_{j=i_{v}}^{l_{v}} w_{y_{v}^{\prime}}^{\prime}\right]\left[\prod_{\substack{v \in V_{d} \\
c_{v}=\alpha_{v}}} \prod_{j=i_{v}}^{l_{v}-1} w_{y_{v}^{j}}^{\prime}\right]
\end{aligned}
$$

Now, for each $r, s$ we distinguish between three cases.

- If $r>0$ (which means $r_{h}=r>0$ and $r_{\bar{h}}=-r<0$ ), then $I_{h}>I_{\bar{h}}$ for any $h, \bar{h}$ in $\mathcal{M}_{r}$. In this case we apply the row inequality (IV.112).

- If $r<0$ (which means $r_{h}=r<0$ and $r_{\bar{h}}=-r>0$ ), then $I_{h}<I_{\bar{h}}$ for any $h, \bar{h}$ in $\mathcal{M}_{r}$. This case is similar to the first case, exchanging the role of fields and antifields, so we apply the column inequality (IV.113).

- If $r=0$ (which means $r_{h}=r=0$ and $r_{\bar{h}}=-r=0$ ), then $I_{h}=I_{\bar{h}}$ for any $h, \bar{h}$ in $\mathcal{M}_{r}$. In this case we must analyze in more detail the subdeterminants as will be explained later.

With these conventions the fixed index (field or antifield) in the sum $\sum_{j=1}^{n}\left|M_{i j}\right|^{2}$ for Hr or $\sum_{i=1}^{n}\left|M_{i j}\right|^{2}$ for Hc is always the one with the highest weight $I$. This is essential in the following bounds.

IV.3.2 Case $r>0($ and $r<0)$

As remarked above we treat only the case $r>0$, the other case being similar, exchanging fields and antifields, hence rows and columns. In that case we apply the row inequality (एV.112):

$$
\left|\operatorname{det} \mathcal{M}_{r, s}\left(\left\{\beta_{h}\right\}\left\{\beta_{\bar{h}}\right\}\right)\right| \leq \prod_{\substack{h \notin b, r_{h}=r \\ s_{h}=s}}\left(\sum_{\substack{\bar{h} \notin b \mid \beta_{h}=\alpha_{\bar{h}}, \alpha_{h}=\beta_{\bar{h}}, k_{\bar{h}}=k_{h}, r_{\bar{h}}=-r, s_{\bar{h}}=-s}}\left|M_{h, \bar{h}}\right|^{2}\right)^{\frac{1}{2}}
$$


where $h \notin b$ is the set of fields that are not extracted from the determinant to give some $h$-link. Now

$$
\begin{aligned}
& \left|M_{h, \bar{h}}\right|^{2}=\left|\sum_{j \in \mathcal{I}_{h} \cap \mathcal{I}_{\bar{h}} \cap J\left(k_{h}\right)} \delta_{k_{h}, k_{\bar{h}}}\left[W_{h}(j)\right]\left[h_{\Delta_{h}^{j}, \Delta_{\bar{h}}^{j}}^{\mathcal{F}_{j}^{h}}(w)\right] C_{\Delta_{h}^{j}, \Delta_{\bar{h}}^{j}}^{j k_{h}}\left(x_{h}, x_{\bar{h}}\right)\left[W_{\bar{h}}(j)\right]\right|^{2} \\
& \leq \delta_{k_{h}, k_{\bar{h}}} \sum_{j \in \mathcal{I}_{h}}\left|C_{\Delta_{h}^{j}, \Delta_{\bar{h}}^{j}}^{j k_{h}}\left(x_{h}, x_{\bar{h}}\right)\right|^{2}
\end{aligned}
$$

where the weakening factors $W_{h}(j), W_{\bar{h}}(j)$ and $h_{\Delta_{h}^{j}, \Delta_{\bar{h}}^{j}}^{\mathcal{F}_{h}^{h}}(w)$ are bounded by one, the sum over $j$ is performed over the larger set $\mathcal{I}_{h} \cap \mathcal{I}_{\bar{h}} \cap J\left(k_{h}\right) \subset \mathcal{I}_{h}$, which is an upper bound, and we applied the identity

$$
C_{\Delta_{h}^{j}, \Delta_{\bar{h}}^{j}}^{j k_{h}}\left(x_{h}, x_{\bar{h}}\right) C_{\Delta_{h}^{j^{\prime}}, \Delta_{\bar{h}}^{j^{\prime}}}^{j^{\prime} k_{h}}\left(x_{h}, x_{\bar{h}}\right)=0 \quad \text { if } j \neq j^{\prime}
$$

which is true by construction. For any $\bar{h}$ in the sum, its weight satisfies

$$
I_{h} 2^{-r} \leq I_{\bar{h}}<I_{h} 2^{-r+1}
$$

Before going on we prove the following lemma

Lemma. If $r>0$, the only non zero contributions are for $\alpha_{h}<5$.

Proof Actually if there exists $\alpha_{h}=5$ we must have

$$
M^{-4 i_{h}}>2^{r-1} I_{\bar{h}} \geq I_{\bar{h}} .
$$

But this is impossible. Indeed let us consider for instance the case $\alpha_{\bar{h}}=1$. Then

$$
M^{-4 i_{v_{h}}}>n_{\Delta_{\bar{h}}} M^{-4 i_{\Delta_{\bar{h}}}} f_{\Delta_{\bar{h}}}^{-1} \geq M^{-4 i_{\Delta_{\bar{h}}}}
$$

which implies $i_{v_{h}}<i_{\Delta_{\bar{h}}}$. But to contract $h$ with $\bar{h}$ we must also have $i_{v_{h}} \geq i_{\Delta_{\bar{h}}}$, which is a contradiction. The other cases are verified in the same way. 
Now the first step is to estimate the sum over $\bar{h}$

$$
\Sigma_{\bar{h}}=\sum_{\left\{\begin{array}{c}
\bar{h} \notin b \mid \beta_{h}=\alpha_{\bar{h}}, \alpha_{h}=\beta_{\bar{h}}, \\
k_{\bar{h}}=k_{h}, r_{\bar{h}}=-r, s_{\bar{h}}=-s
\end{array}\right\}}\left|M_{h, \bar{h}}\right|^{2}
$$

For this purpose we distinguish five cases.

1.) $\beta_{h}=1$ which means that $h$ can contract only with $\bar{h}$ of type $1\left(\alpha_{\bar{h}}=1\right)$. Therefore for any $\bar{h}$ the weight $I$ is

$$
I_{\bar{h}}=n_{\Delta_{\bar{h}}} M^{-4 i_{\Delta_{\bar{h}}}} f_{\Delta_{\bar{h}}}^{-1}
$$

Therefore the sum $\Sigma_{\bar{h}}$ is bounded by

$$
\Sigma_{\bar{h}} \leq \sum_{j \in \mathcal{I}_{h}} \sum_{\Delta \in \mathcal{D}_{j}}\left|C^{j k_{h}}\left(x_{\Delta_{h}^{j}}, x_{\Delta}\right)\right|^{2} \sum_{\Delta^{\prime} \in B_{S}, \Delta^{\prime} \subset \Delta} 2 n_{\Delta^{\prime}}
$$

where $2 n_{\Delta^{\prime}}$ is the maximal number of antifields (two for each vertex) localized in $\Delta^{\prime}$. We remark that the vertex position in the propagator is substituted by the cube center $x_{\Delta}$. By ([V.130) and ([V.134) we see that

$$
n_{\Delta^{\prime}}<I_{h} 2^{-r+1} M^{4 i_{\Delta^{\prime}}} f_{\Delta^{\prime}}
$$

therefore (ㅍ.135) is bounded by

$$
\Sigma_{\bar{h}} \leq 2 I_{h} 2^{-r+1} \sum_{j \in \mathcal{I}_{h}} \sum_{\Delta \in \mathcal{D}_{j}}\left|C^{j k_{h}}\left(x_{\Delta_{h}^{j}}, x_{\Delta}\right)\right|^{2} \sum_{\Delta^{\prime} \in B_{S}, \Delta^{\prime} \subset \Delta} M^{4 i_{\Delta^{\prime}}} f_{\Delta^{\prime}}
$$

Now we observe that $M^{4 i} i_{\Delta^{\prime}} f_{\Delta^{\prime}}$ is the exposed volume of $\Delta^{\prime}$ and that, for any cube $\Delta, \cup_{\Delta^{\prime} \subset \Delta} E x\left(\Delta^{\prime}\right)$ is a partition of $\Delta$. Therefore we have

$$
\sum_{\Delta^{\prime} \in B_{S}, \Delta^{\prime} \subset \Delta} M^{4 i \Delta^{\prime}} f_{\Delta^{\prime}}=M^{4 j}
$$

hence $\Sigma_{\bar{h}}$ is bounded by

$$
\Sigma_{\bar{h}} \leq I_{h} 2^{-r+2} \sum_{j \in \mathcal{I}_{h}} M^{4 j} \sum_{\Delta \in \mathcal{D}_{j}}\left|C^{j k_{h}}\left(x_{\Delta_{h}^{j}}, x_{\Delta}\right)\right|^{2}
$$


Finally the sum over $\Delta$ is bounded by

$$
\sum_{\Delta \in \mathcal{D}_{j}}\left|C^{j k_{h}}\left(x_{\Delta_{h}^{j}}, x_{\Delta}\right)\right|^{2} \leq C M^{\frac{16}{3}} M^{-4 j} M^{-\frac{4}{3} k_{h}} \sum_{\Delta \in \mathcal{D}_{j}} \chi_{j, k}\left(x_{\Delta_{h}^{j}}, x_{\Delta}\right)
$$

where from now on we use $C$ as generic name for a constant independent of $M$ which can be tracked but whose numerical precise value is inessential. We applied the scaled decay ([I.42)-([I.46), and the function $\chi_{j, k}$ is different from zero only for $\left|\vec{x}_{\Delta_{h}^{j}}-\vec{x}_{\Delta}\right| \leq M^{j}$ and $\left|t_{\Delta_{h}^{j}}-t_{\Delta}\right| \leq M^{j+k}$ (actually for $k>0$ we have $\left.\left|\vec{x}_{\Delta_{h}^{j}}-\vec{x}_{\Delta}\right| \simeq M^{j-\frac{k}{3}+\frac{1}{3}} \leq M^{j}\right)$. Now, for $x_{\Delta_{h}^{j}}$ fixed, the number of cubes such that their center $x_{\Delta}$ satisfies these bounds is at most $26\left(2 M^{k_{h}}\right)$ where 26 is the number of nearest neighbors of $\Delta_{h}^{j}$ in the position space, and $2 M^{k_{h}}$ is the number of choices in the time direction. Therefore

$$
\sum_{\Delta \in \mathcal{D}_{j}}\left|C^{j k_{h}}\left(x_{\Delta_{h}^{j}}, x_{\Delta}\right)\right|^{2} \leq C M^{\frac{16}{3}} M^{-4 j} M^{-\frac{k_{h}}{3}}
$$

Remark that the case $j=j_{M}+1$ needs a different treatment. Actually in this case we have

$$
\begin{aligned}
& \sum_{\Delta \in \mathcal{D}_{j}}\left|C^{j_{M}+1,0}\left(x_{\Delta}, x_{\Delta^{\prime}}\right)\right|^{2} \leq C_{p} M^{-4 j_{M}} \sum_{\Delta \in \mathcal{D}_{j}} \frac{\chi\left(\left|t_{\Delta}-t_{\Delta^{\prime}}\right| \leq M^{j_{M}}\right)}{\left(1+M^{-j_{M}}\left|\vec{x}_{\Delta}-\vec{x}_{\Delta^{\prime}}\right|\right)^{2 p}} \\
& \leq M^{-4 j_{M}} \sum_{n_{1}, n_{2}, n_{3} \in \mathbb{Z}} \frac{C_{p}}{\left(1+M^{-j_{M}} M^{j_{M}}\left(\left|n_{1}\right|+\left|n_{2}\right|+\left|n_{3}\right|\right)\right)^{2 p}} \leq C M^{-4 j_{M}} .
\end{aligned}
$$

The sum $\Sigma_{\bar{h}}$ is finally bounded by

$$
\begin{aligned}
\Sigma_{\bar{h}} & \leq C M^{\frac{16}{3}} I_{h} 2^{-r} \sum_{j \in \mathcal{I}_{h}} M^{4 j} M^{-4 j} M^{-\frac{k_{h}}{3}} \leq C M^{\frac{16}{3}} I_{h} 2^{-r} M^{-\frac{k_{h}}{3}}\left|\mathcal{I}_{h}\right| \\
& \leq C M^{\frac{16}{3}} I_{h} 2^{-r} M^{-\frac{k_{h}}{3}} j_{M}
\end{aligned}
$$

where $\left|\mathcal{I}_{h}\right|$ is the number of elements in the interval $\mathcal{I}_{h}$, the numerical constants have been absorbed in $C$ and we bounded $\left|\mathcal{I}_{h}\right|$ by $j_{M}$.

2.) $\beta_{h}=2$ which means that $h$ can contract only with $\bar{h}$ of type $2\left(\alpha_{\bar{h}}=2\right)$. Therefore all $\bar{h}$ must be hooked to some vertex in $V_{d}$ and must have scale attribution $i_{v_{\bar{h}}} \leq j_{\bar{h}} \leq l_{v_{\bar{h}}}$. The weight $I_{\bar{h}}$ is

$$
I_{\bar{h}}=M^{-4 i_{v_{\bar{h}}}}=M^{-4 i_{r}(h)}
$$


where $i_{r}(h)$ is the unique scale for which (IV.130) is satisfied. Now

$$
\Sigma_{\bar{h}} \leq \sum_{\substack{j \in \mathcal{I}_{h} \\ j \geq i_{r}(h)}} \sum_{\Delta \in \mathcal{D}_{j}}\left|C^{j k_{h}}\left(x_{\Delta_{h}^{j}}, x_{\Delta}\right)\right|^{2} 2\left(j_{M}+2-j\right)
$$

where $j_{M}+2-j \leq 2 j_{M}$ is the maximal number of cubes at scale $j_{M}+1 \geq j^{\prime} \geq$ $j$ containing $\Delta$. As $j=j_{\bar{h}} \leq l_{v_{\bar{h}}}$, only vertices localized in these cubes can contribute. The factor 2 appears because there is only one vertex localized in each cube and at most 2 antifields hooked to that vertex. The sum over $\Delta$ is performed as in the case 1.). Therefore

$$
\Sigma_{\bar{h}} \leq C j_{M} M^{\frac{16}{3}} M^{-\frac{k_{h}}{3}} \sum_{j \in \mathcal{I}_{h}} M^{-4 j}
$$

Now

$$
M^{-4 j}=M^{-4\left(j-i_{r}(h)\right)} M^{-4 i_{r}(h)} \leq M^{-4\left(j-i_{r}(h)\right)} 2^{-r+1} I_{h}
$$

The sum over $j$ is performed with the decay $M^{-4\left(j-i_{r}(h)\right)}$

$$
\sum_{\substack{j \in \mathcal{I}_{h} \\ j \geq i_{r}(h)}} M^{-4\left(j-i_{r}(h)\right)} \leq C
$$

Finally

$$
\Sigma_{\bar{h}} \leq C M^{\frac{16}{3}} I_{h} 2^{-r} M^{-\frac{k_{h}}{3}} j_{M}
$$

where all constants have been inserted in $C$.

3.) $\beta_{h}=3$ which means that $h$ can contract only with $\bar{h}$ of type $3\left(\alpha_{\bar{h}}=3\right)$. Therefore all $\bar{h}$ in the sum are hooked to some $v \in V_{d}$ and have $j_{\bar{h}}>l_{v}$. The weight is

$$
I_{\bar{h}}=M^{-4 l_{\bar{h}}}=M^{-4 i_{r}(h)}
$$

where $i_{r}(h)$ is the unique scale for which (IV.130) is satisfied. Then

$$
\Sigma_{\bar{h}} \leq \sum_{j \in \mathcal{I}_{h}} \sum_{\Delta \in \mathcal{D}_{j}}\left|C^{j k_{h}}\left(x_{\Delta_{h}^{j}}, x_{\Delta}\right)\right|^{2} \sum_{\Delta^{\prime} \in \mathcal{D}_{i_{r}(h)}, \Delta^{\prime} \subset \Delta} 2
$$


where 2 is the maximal number of antifields with $\Delta_{v} \subseteq \Delta^{\prime}$ that are hooked to the vertex $v_{l}$ of the vertical link $l \in v L_{i_{\Delta^{\prime}}}$ connecting the connected component $y_{k}^{j}\left(j=i_{\Delta^{\prime}}\right)$ containing $\Delta^{\prime}$ to its ancestor. Now we observe that

$$
\sum_{\Delta^{\prime} \in \mathcal{D}_{i_{r}(h)}, \Delta^{\prime} \subset \Delta} 2 \leq 2 M^{4 j} M^{-4 i_{r}(h)}
$$

where $M^{4 j-4 i_{r}(h)}$ is the number of cubes of scale $i_{r}(h)$ contained in a cube of

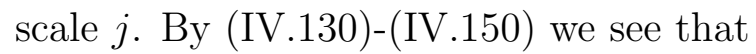

$$
M^{-4 i_{r}(h)} \leq I_{h} 2^{-r+1}
$$

hence $\Sigma_{\bar{h}}$ is bounded by

$$
\Sigma_{\bar{h}} \leq 2 I_{h} 2^{-r+1} \sum_{j \in \mathcal{I}_{h}} M^{4 j} \sum_{\Delta \in \mathcal{D}_{j}}\left|C^{j k_{h}}\left(x_{\Delta_{h}^{j}}, x_{\Delta}\right)\right|^{2}
$$

The sum over $\Delta$ is bounded as in the case 1.) above. Therefore

$$
\begin{aligned}
\Sigma_{\bar{h}} & \leq C M^{\frac{16}{3}} I_{h} 2^{-r} \sum_{j \in \mathcal{I}_{h}} M^{4 j} M^{-4 j} M^{-\frac{k_{h}}{3}} \leq C M^{\frac{16}{3}} I_{h} 2^{-r} M^{-\frac{k_{h}}{3}}\left|\mathcal{I}_{h}\right| \\
& \leq C M^{\frac{16}{3}} I_{h} 2^{-r} M^{-\frac{k_{h}}{3}} j_{M}
\end{aligned}
$$

where $\left|\mathcal{I}_{h}\right| \leq j_{M}+1 \leq 2 j_{M}$ and all constant factors are absorbed in $C$.

4.) $\beta_{h}=4$ which means that $h$ can contract only with $\bar{h}$ of type $4\left(\alpha_{\bar{h}}=4\right)$. Therefore all $\bar{h}$ in the sum are associated to some $f$-link of order 6 and its weight is

$$
I_{\bar{h}}=M^{-4 i_{\bar{h}}}=M^{-4 i_{r}(h)}
$$

where $i_{r}(h)$ is the unique scale for which (IV.130) is satisfied. Then

$$
\Sigma_{\bar{h}} \leq \sum_{j \in \mathcal{I}_{h}} \sum_{\Delta \in \mathcal{D}_{j}}\left|C^{j k_{h}}\left(x_{\Delta_{h}^{j}}, x_{\Delta}\right)\right|^{2} \sum_{\Delta^{\prime} \in \mathcal{D}_{i_{r}(h)}, \Delta^{\prime} \subset \Delta} 6
$$

where 6 is the maximal number of antifields with $\Delta_{v} \subset \Delta^{\prime}$ that have been derived by a $f$-link of order 6 at scale $i_{\Delta^{\prime}}$ for the connected component $y_{k}^{j}$ $\left(j=i_{\Delta^{\prime}}\right)$ containing $\Delta^{\prime}$. Now we can apply the same analysis as for the case 3.) except that instead of a factor 2 we have a factor 6 . Hence we obtain

$$
\Sigma_{\bar{h}} \leq C M^{\frac{16}{3}} I_{h} 2^{-r} M^{-\frac{k_{h}}{3}} j_{M}
$$


5.) $\beta_{h}=5$ which means that $h$ can contract only with $\bar{h}$ of type $5\left(\alpha_{\bar{h}}=5\right)$. Therefore all $\bar{h}$ in the sum are hooked to some $v \in V_{d}$ and have $j_{\bar{h}}=i_{v}$. The weight is

$$
I_{\bar{h}}=M^{-4 i_{\bar{h}}}=M^{-4 i_{r}(h)}
$$

where $i_{r}(h)$ is the unique scale for which ([V.130) is satisfied. There is no sum over $j$ to compute, as we have only $j=i_{r}(h)$.

$$
\Sigma_{\bar{h}} \leq \sum_{\Delta \in \mathcal{D}_{i_{r}(h)}} \mid C^{i_{r}(h) k_{h}}\left(\left.x_{\left.\Delta_{h}^{i_{r}(h)}, x_{\Delta}\right)}\right|^{2} n(\bar{h})\right.
$$

We know that $s$ is negative, and by (IV.123) (and the fact that $n(h)=1$ ), we obtain $n(\bar{h}) \leq 2^{-s}=2^{|s|}$. Therefore

$$
\Sigma_{\bar{h}} \leq 2^{|s|} \sum_{\Delta \in \mathcal{D}_{i_{r}(h)}}\left|C^{i_{r}(h) k_{h}}\left(x_{\Delta_{h}^{i_{r}(h)}}, x_{\Delta}\right)\right|^{2}
$$

The sum over $\Delta$ is performed as in the other cases then

$$
\Sigma_{\bar{h}} \leq C 2^{|s|} M^{\frac{16}{3}} M^{-\frac{k_{h}}{3}} M^{-4 i_{r}(h)}
$$

Applying (IV.159) we have

$$
\Sigma_{\bar{h}} \leq C 2^{|s|} 2^{-r} I_{h} M^{\frac{16}{3}} M^{-\frac{k_{h}}{3}}
$$

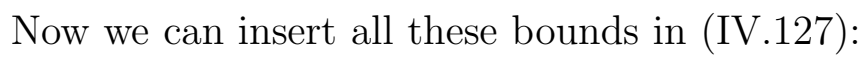

$$
\begin{aligned}
\left|\operatorname{det} \mathcal{M}_{r, s}\left(\left\{\beta_{h}\right\}\left\{\beta_{\bar{h}}\right\}\right)\right| \leq & \left(C M^{\frac{8}{3}}\right)^{n_{r, s}} \prod_{\substack{h \notin b, r_{h}=r \\
s_{h}=s, \beta_{h}=1, \ldots, 4}}\left[I_{h}^{\frac{1}{2}} 2^{-\frac{r}{2}} j_{M}^{\frac{1}{2}} M^{-\frac{k_{h}}{6}}\right] \\
& \prod_{\substack{h \notin b, r_{h}=r \\
s_{h}=s, \beta_{h}=5}}\left[I_{h}^{\frac{1}{2}} 2^{-\frac{r}{2}} 2^{\frac{|s|}{2}} M^{-\frac{k_{h}}{6}}\right] \quad \text { (IV.164) }
\end{aligned}
$$

where $C$ is a constant and $n_{r, s}$ is the number of fields belonging to the matrix $\mathcal{M}_{r, s}$. Now we observe that

$$
\begin{aligned}
& \prod_{\substack{h \notin b, r_{h}=r \\
s_{h}=s, \beta h=\beta}}\left[I_{h}^{\frac{1}{2}} 2^{-\frac{r}{2}}\right] \leq \prod_{\substack{h \notin b, r_{h}=r \\
s_{h}=s, \beta_{h}=\beta}}\left[I_{h}^{\frac{1}{4}} 2^{\frac{r}{4}} 2^{-\frac{r}{2}}\right] \prod_{\substack{\bar{h} \notin b, r \\
\bar{h}=-r \\
s_{\bar{h}}=-s, \alpha_{\bar{h}}=\beta}}\left[I_{\bar{h}}^{\frac{1}{4}}\right] \\
& =\prod_{\substack{h \notin b, r_{h}=r \\
s_{h}=s, \beta_{h}=\beta}}\left[I_{h}^{\frac{1}{4}} 2^{-\frac{r}{8}}\right] \prod_{\substack{\bar{h} \notin b, r_{\bar{h}}=-r \\
s \bar{h}=-s, \alpha_{\bar{h}}=\beta}}\left[I_{\bar{h}}^{\frac{1}{4}} 2^{-\frac{r}{8}}\right]
\end{aligned}
$$


where we applied the relation (IV.130) and the fact that $\mid\left\{h \mid r_{h}=r, s_{h}=\right.$ $\left.s, \beta_{h}=\beta\right\}|=|\left\{\bar{h} \mid r_{\bar{h}}=-r, s_{\bar{h}}=-s, \alpha_{\bar{h}}=\beta\right\} \mid$ for $\beta=1, \ldots, 5$. Moreover

$$
\prod_{\left\{\begin{array}{c}
h \notin b, r_{h}=r \\
s_{h}=s
\end{array}\right\}}\left[M^{-\frac{k_{h}}{6}}\right]=\prod_{\substack{h \notin b, r_{h}=r \\
s_{h}=s}}\left[M^{-\frac{k_{h}}{12}}\right] \prod_{\substack{\bar{h} \notin b, r_{\bar{h}}=-r \\
s \bar{h}=-s}}\left[M^{-\frac{k_{\bar{h}}}{12}}\right]
$$

since $\left|\left\{h \mid r_{h}=r, s_{h}=s, k_{h}=k\right\}\right|=\left|\left\{\bar{h} \mid r_{\bar{h}}=-r, s_{\bar{h}}=-s, k_{\bar{h}}=k\right\}\right|$ for any $k \geq 0$ and

$$
\begin{aligned}
& \prod_{\substack{h \notin b, r_{h}=r \\
s_{h}=s, \beta_{h}=1, \ldots, 4}}\left[j_{M}^{\frac{1}{2}}\right]=\prod_{\substack{h \notin b, r_{h}=r \\
s_{h}=s, \beta_{h}=1, \ldots, 4}}\left[j_{M}^{\frac{1}{4}}\right] \prod_{\substack{\bar{h} \notin b, r_{\bar{h}}=-r \\
s_{\bar{h}}=-s, \alpha_{\bar{h}}=1, \ldots, 4}}\left[j^{\frac{1}{4}}\right] \\
& \leq \prod_{\left\{\begin{array}{c}
h \notin b, r_{h}=r \\
s_{h}=s, \alpha_{h}=1, \ldots, 4
\end{array}\right\}}\left[j_{M}^{\frac{1}{4}}\right] \prod_{\substack{\bar{h} \notin b, r_{\bar{h}}=-r \\
s_{\bar{h}}=-s, \alpha_{\bar{h}}=1, \ldots, 4}}\left[j_{M}^{\frac{1}{4}}\right]
\end{aligned}
$$

where we applied the relation $\left|\left\{h \mid r_{h}=r, s_{h}=s, \alpha_{h}=1, \ldots, 4\right\}\right|=\mid\left\{\bar{h} \mid r_{\bar{h}}=\right.$ $\left.-r, s_{\bar{h}}=-s, \alpha_{\bar{h}}=1, \ldots, 4\right\}|+|\left\{\bar{h} \mid r_{\bar{h}}=-r, s_{\bar{h}}=-s, \alpha_{\bar{h}}=5\right\} \mid$ which is true because $\alpha_{h}<5 \forall h$. Now, for any $h$ with $\beta_{h}=5$ there is no factor $j_{M}$ therefore we write $1 \leq j_{M}^{\frac{1}{4}}$.

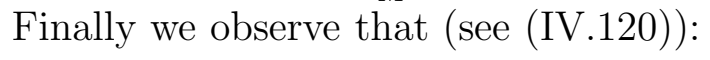

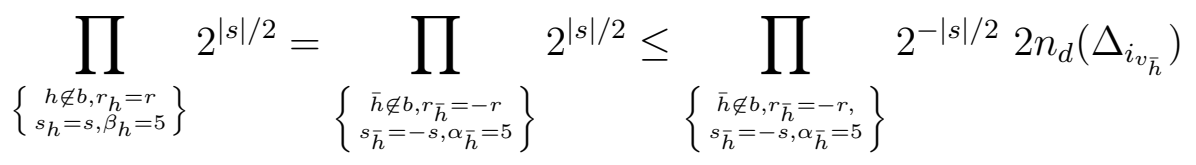

$$
\begin{aligned}
& \leq \prod_{\left\{\begin{array}{c}
h \notin b, r_{h}=r, \\
s \\
s_{h}=s
\end{array}\right\}} 2^{-|s| / 4} \prod_{\substack{\bar{h} \notin b, r_{h}=-r, h \\
s_{\bar{h}}=-s, \alpha_{\bar{h}}=5}} 2^{-|s| / 4} 2 n_{d}\left(\Delta_{i_{v_{\bar{h}}}}\right)
\end{aligned}
$$

where we apply the inequality $2^{|s|} \leq 2 n_{d}\left(\Delta_{i_{v_{\bar{h}}}}\right)$. The determinant $\left|\operatorname{det} \mathcal{M}_{r, s}\right|$ is then bounded by

$$
\begin{gathered}
\left|\operatorname{det} \mathcal{M}_{r, s}\right| \leq\left(C M^{\frac{8}{3}}\right)^{n_{r, s}} \prod_{\substack{h \notin b, r_{h}=r \\
s_{h}=s, \alpha_{h}<5}}\left[I_{h}^{\frac{1}{4}} 2^{-\frac{r}{8}} 2^{-\frac{|s|}{4}} M^{-\frac{k_{h}}{12}} j_{M}^{\frac{1}{4}}\right] \\
\prod_{\substack{\bar{h} \notin b, r_{\bar{h}}=-r \\
s \bar{h}=-s, \alpha_{\bar{h}}<5}}\left[I_{\bar{h}}^{\frac{1}{4}} 2^{-\frac{r}{8}} 2^{-\frac{|s|}{4}} j_{M}^{\frac{1}{4}} M^{-\frac{k_{\bar{h}}}{12}}\right]
\end{gathered}
$$




$$
\prod_{\left\{\begin{array}{c}
\bar{h} \notin b, r, r_{\bar{h}}=-r \\
s \bar{h}=-s, \alpha_{\bar{h}}=5
\end{array}\right\}}\left[I_{\bar{h}}^{\frac{1}{4}} 2^{-\frac{r}{8}} 2^{-\frac{|s|}{4}} M^{-\frac{k_{\bar{h}}}{12}} n_{d}\left(\Delta_{i_{\bar{h}}}\right)\right]
$$

where $n_{r, s}$ is the number of fields in the determinant. Inserting the definitions for $I$ we can write the determinant as

$$
\begin{aligned}
& \left|\operatorname{det} \mathcal{M}_{r, s}\right| \leq\left(C M^{\frac{8}{3}}\right)^{n_{r, s}} \prod_{\substack{h \notin b, r_{h}=r \\
s_{h}=s, \alpha_{h}=1}}\left[M^{-i_{\Delta_{h}}} n_{\Delta_{h}}^{\frac{1}{4}} \frac{1}{f_{\Delta_{h}}^{\frac{1}{4}}} 2^{-\frac{r}{8}} 2^{-\frac{|s|}{4}} M^{-\frac{k_{h}}{12}} j_{M}^{\frac{1}{4}}\right] \\
& \prod_{\left\{\begin{array}{c}
h \notin b, r, r_{h}=r \\
s_{h}=s, \alpha_{h}=2
\end{array}\right\}}\left[M^{-i_{v_{h}}} 2^{-\frac{r}{8}} 2^{-\frac{|s|}{4}} M^{-\frac{k_{h}}{12}} j_{M}^{\frac{1}{4}}\right] \prod_{\substack{h \notin b, r_{h}=r \\
s_{h}=s, \alpha_{h}=3}}\left[M^{-l_{v_{h}}} 2^{-\frac{r}{8}} 2^{-\frac{|s|}{4}} M^{-\frac{k_{h}}{12}} j_{M}^{\frac{1}{4}}\right] \\
& \prod_{\substack{h \notin b, r_{h}=r \\
s_{h}=s, \alpha_{h}=4}}\left[M^{-i_{h}} 2^{-\frac{r}{8}} 2^{-\frac{|s|}{4}} M^{-\frac{k_{h}}{12}} j_{M}^{\frac{1}{4}}\right] \\
& \prod_{\left\{\begin{array}{c}
\bar{h} \notin b, r \bar{h}=-r \\
s_{\bar{h}}=-s, \alpha_{\bar{h}}=1
\end{array}\right\}}\left[M^{-i_{\Delta_{\bar{h}}}} n_{\Delta_{\bar{h}}^{\frac{1}{4}}} \frac{1}{f_{\Delta_{\bar{h}}}^{\frac{1}{4}}} 2^{-\frac{r}{8}} 2^{-\frac{|s|}{4}} M^{-\frac{k_{\bar{h}}}{12}} j_{M}^{\frac{1}{4}}\right]
\end{aligned}
$$

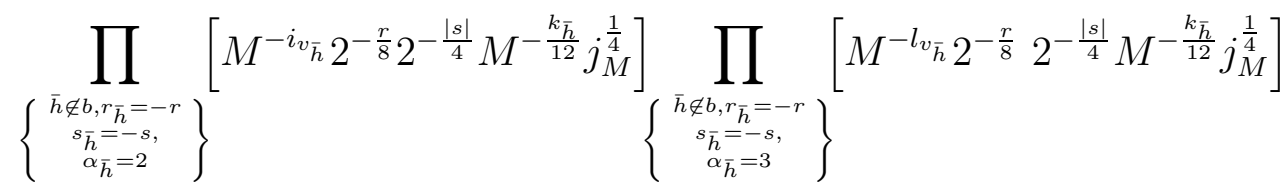

$$
\begin{aligned}
& \prod_{\left\{\begin{array}{c}
\bar{h} \notin b, r_{\bar{h}}=-r \\
s_{\bar{h}}=-s, \alpha_{\bar{h}}=4
\end{array}\right\}}\left[M^{-i_{\bar{h}}} 2^{-\frac{r}{8}} 2^{-\frac{|s|}{4}} M^{-\frac{k_{\bar{h}}}{12}} j_{M}^{\frac{1}{4}}\right] \\
& \prod_{\left\{\begin{array}{c}
\bar{h} \notin b, r \bar{h}=-r \\
s_{\bar{h}}=-s, \alpha_{\bar{h}}=5
\end{array}\right\}}\left[M^{-i_{v_{\bar{h}}}} 2^{-\frac{r}{8}} 2^{-\frac{|s|}{4}} M^{-\frac{k_{\bar{h}}}{12}} n_{d}\left(\Delta_{i_{\bar{h}}}\right)\right]
\end{aligned}
$$

where we applied the fact that for $r>0, \alpha_{h}<5 \forall h$.

\section{IV.3.3 Case $r=0$}

The subdeterminant for $r=0$ actually needs a more detailed analysis. We can write it as

$$
\begin{aligned}
& \operatorname{det} \mathcal{M}_{0, s}\left(\left\{\beta_{h}\right\}\left\{\beta_{\bar{h}}\right\}\right)=\operatorname{det} \mathcal{M}_{0, s}(\leq 5,<5) \\
& \operatorname{det} \mathcal{M}_{0, s}(<5,=5) \operatorname{det} \mathcal{M}_{0, s}(=5,=5)
\end{aligned}
$$


where the first subdeterminant contains contractions between fields with $\alpha_{h}<5$ (which corresponds to $\beta_{\bar{h}}<5$ ) and any antifield (which corresponds to $\left.\beta_{h} \leq 5\right)$, the second subdeterminant contains contractions between fields with $\alpha_{h}=5$ (which corresponds to $\beta_{\bar{h}}=5$ ) and antifields with $\alpha_{\bar{h}}<5$ (which corresponds to $\beta_{h}<5$ ). Finally the third subdeterminant contains contractions between fields with $\alpha_{h}=5$ (which corresponds to $\beta_{\bar{h}}=5$ ) and antifields with $\alpha_{\bar{h}}=5$ (which corresponds to $\beta_{h}=5$ ).

In the first case $\left(\alpha_{h}<5\right)$ we apply exactly the same bound as for $r>0$. In the second case $\left(\alpha_{h}=5, \beta_{h}<5\right)$ we apply the column inequality (IV.112) and everything goes as in the case $r>0$ exchanging fields and antifields.

Finally in the third case we have some field with $\alpha_{h}=5$ contracting with some antifield with $\alpha_{\bar{h}}=5$. Here again we optimize the Hadamard inequalities depending on the sign of $s$. If $s \geq 0$ we apply the row inequality, and symmetricallyf?

The two main weights are equal

$$
I_{\bar{h}}=M^{-4 i_{\bar{h}}}=M^{-4 i_{v_{h}}}=I_{h}
$$

Remark that there is no sum over $j$ to compute, as we have only $j=i_{v_{h}}$.

$$
\Sigma_{\bar{h}} \leq \sum_{\Delta \in \mathcal{D}_{i_{v_{h}}}}\left|C^{i_{v_{h}} k_{h}}\left(x_{\Delta_{h}^{i_{h}}}, x_{\Delta}\right)\right|^{2} n(\bar{h})
$$

We know that $n(\bar{h})<2^{-s+1} n(h)$ therefore

$$
\Sigma_{\bar{h}} \leq 2^{-|s|+1} n(h) \sum_{\Delta \in \mathcal{D}_{i_{v_{h}}}} \mid C^{i_{r}(h) k_{h}}\left(\left.x_{\left.\Delta_{h}^{i_{r}(h)}, x_{\Delta}\right)}\right|^{2}\right.
$$

The sum over $\Delta$ is performed as in the other cases and we get

$$
\Sigma_{\bar{h}} \leq 2^{-|s|+1} n\left(\Delta_{v_{h}}\right) C M^{\frac{16}{3}} M^{-\frac{k_{h}}{3}} M^{-4 i_{v_{h}}}=C 2^{-|s|} n\left(\Delta_{v_{h}}\right) I_{h} M^{\frac{16}{3}} M^{-\frac{k_{h}}{3}}
$$

where the constant 2 has been inserted into $C$. As before we will distribute the factor $2^{-|s|}$ on both sides of the determinant which gives again factors $2^{-|s| / 4}$ for each field or antifield of this determinant after the Hadamard inequality. The other factors are unchanged.

\footnotetext{
${ }^{9}$ This second optimization is not really necessary, but nicer.
} 


\section{IV.3.4 Result of the weight expansion}

The global determinant is bounded by

$$
\begin{aligned}
& \prod_{r, s}\left|\operatorname{det} \mathcal{M}_{r, s}\right| \leq C^{n} M^{\frac{16 n}{3}} \prod_{h \notin b} 2^{-\frac{\left|r_{h}\right|}{8}-\frac{\left|s_{h}\right|}{4}} M^{-\frac{k_{h}}{12}} \prod_{\bar{h} \notin b} 2^{-\frac{\left|r_{\bar{h}}\right|}{8}-\frac{\left|s_{\bar{h}}\right|}{4}} M^{-\frac{k_{\bar{h}}}{12}} \\
& \prod_{\substack{h \notin b, \alpha_{h}=1}}\left[M^{-i_{\Delta_{h}}} n_{\Delta_{h}}^{\frac{1}{4}} f_{\Delta_{h}}^{-\frac{1}{4}} j_{M}^{\frac{1}{4}}\right] \prod_{\substack{h \notin b, \alpha_{h}=2,3}}\left[M^{-i_{v_{h}}} j_{M}^{\frac{1}{4}}\right] \prod_{\substack{h \notin b, \alpha_{h}=4}}\left[M^{-i_{h}} j_{M}^{\frac{1}{4}}\right] \\
& \prod_{\substack{h \notin b, \alpha \notin h \\
\alpha_{h}=5}}\left[M^{-i_{v_{h}}} n_{d}\left(\Delta_{i_{v_{h}}}\right)\right] \prod_{\substack{\bar{h} \notin b, \alpha_{\bar{h}}=1}}\left[M^{-i_{\Delta_{\bar{h}}}} n_{\Delta_{\bar{h}}}^{\frac{1}{4}} f_{\Delta_{\bar{h}}}^{-\frac{1}{4}} j_{M}^{\frac{1}{4}}\right] \prod_{\substack{\bar{h} \notin b, \alpha_{\bar{h}}=2,3}}\left[M^{-i_{v_{\bar{h}}}} j_{M}^{\frac{1}{4}}\right] \\
& \prod_{\left\{\begin{array}{cc}
\bar{h} \notin b, \\
\alpha \bar{h}=4
\end{array}\right\}}\left[M^{-i_{\bar{h}}} j_{M}^{\frac{1}{4}}\right] \prod_{\substack{\bar{h} \notin b, \alpha \bar{h}=5}}\left[M^{-i_{v_{\bar{h}}}} n_{d}\left(\Delta_{i_{\bar{h}}}\right)\right]
\end{aligned}
$$

where we have applied $\sum_{r, s} n_{r, s} \leq 2 n$, and all numerical factors have been absorbed in the constant $C$. The factors $M^{-l_{v_{h}}}$ have been moreover bounded by $M^{-i_{v_{h}}}$. Inserting this result inside (IV.126) we have

$$
\begin{aligned}
& \sum_{\substack{Y \\
0 \in Y}}\left|A_{c}(Y)\right| L^{|Y|} \leq \sum_{M_{Y}} \sum_{S} \sum_{V L} L^{|Y|} \sum_{B_{S}} \sum_{\left\{x_{\Delta}\right\}^{c}} \sum_{n=0}^{\infty} \frac{|\lambda|^{n}\left(C M^{\frac{16}{3}}\right)^{n}}{n !} \sum_{V_{d}, \alpha_{V_{d}}} \sum_{a, b, R} \\
& \sum_{\left\{v_{l}\right\}_{l \in v L}} \sum_{n_{V_{d}} \sigma_{V_{d}} \rho_{V_{d}}} \sum_{\left\{n_{\Delta}\right\}_{\Delta \in B_{S}}} \sum_{\Delta_{V_{d}}^{c}}\left[\prod_{v \in V_{d}} \sum_{i_{v} \in I_{v}^{c}} \sum_{\Delta_{v} \in \mathcal{D}_{i_{v}} \cap Y}\right] \sum_{\left\{J_{h}^{a}\right\},\left\{J_{h}^{a}\right\}} \sum_{\left\{j_{h}^{b}\right\},\left\{j_{h}^{b}\right\}} \sum_{\left\{k_{h}\right\},\left\{k_{\bar{h}}\right\}} \\
& \left(\prod_{j=m_{Y}}^{M_{Y}} \prod_{k=1}^{c_{j}} \sum_{T_{j k}}\right)\left(\prod_{v \in V_{d}} \int_{\Delta_{v}} d x_{v}\right)\left(\prod_{v \in V_{b} \backslash V_{d}} \int_{E x\left(\Delta_{v}\right)} d x_{v}\right)\left(\prod_{v \in \bar{V}_{d} \backslash V_{b}} M^{4 i_{\Delta_{v}}}\right) \\
& {\left[\prod_{j=m_{Y}}^{M_{Y}} \prod_{k=1}^{c_{j}}\left[\prod_{l \in T_{j k}}\left|C_{\Delta_{l} \bar{\Delta}_{l}}^{j k_{l}}\left(x_{l}, \bar{x}_{l}\right)\right|\right] \delta_{k_{h_{l}} k_{\bar{h}_{l}}}\right] \sum_{\left\{r_{h}\right\},\left\{r_{\bar{h}}\right\}} \sum_{\left\{s_{h}\right\},\left\{s_{\bar{h}}\right\}}} \\
& {\left[\prod_{\{h \notin b\}} 2^{-\frac{\left|r_{h}\right|}{8}-\frac{\left|s_{h}\right|}{4}} \prod_{\{\bar{h} \notin b\}} 2^{-\frac{\left|r_{\bar{h}}\right|}{8}-\frac{\left|s_{\bar{h}}\right|}{4}}\right]\left[\prod_{j=m_{Y}+1}^{M_{Y}}\left(\prod_{l \in v L_{j}} \int_{0}^{1} d w_{l}^{\prime}\right)\right]\left[\prod_{\substack{v \in V_{d} \\
c_{v} \neq \alpha_{v}}}^{\prod_{j=i_{v}}} w_{y_{v}^{j}}^{\prime}\right]}
\end{aligned}
$$




$$
\begin{aligned}
& {\left[\prod_{\substack{v \in V_{d} \\
c_{v}=\alpha_{v}}} \prod_{j=i_{v}}^{l_{v}-1} w_{y_{v}^{j}}^{\prime}\right]\left[\prod_{\{h \notin b\}}\left[M^{-\frac{k_{h}}{12}}\right] \prod_{\{\bar{h} \notin b\}}\left[M^{-\frac{k_{\bar{h}}}{12}}\right]\right] \prod_{v \in V_{d} \cup V_{b}}|\ln T|^{3 / 4}} \\
& \prod_{v \notin V_{d} \cup V_{b}}|\ln T| \sum_{\left\{\beta_{h}\right\}\left\{\beta_{\bar{h}}\right\}}\left[\prod_{\substack{h \notin b \\
\alpha_{h}=1}}\left[M^{-i_{\Delta_{h}}} n_{\Delta_{h}}^{\frac{1}{4}} f_{\Delta_{h}}^{-\frac{1}{4}}\right] \prod_{\substack{\bar{h} \notin b, \alpha_{\bar{h}}=1}}\left[M^{-i_{\Delta_{\bar{h}}}} n_{\Delta_{\bar{h}}}^{\frac{1}{4}} f_{\Delta_{\bar{h}}}^{-\frac{1}{4}}\right]\right] \\
& {\left[\prod_{\substack{h \notin b, \alpha_{h}=2,3}} M^{-i_{v_{h}}} \prod_{\substack{\bar{h} \notin b, \alpha_{\bar{h}}=2,3}} M^{-i_{\bar{h}}}\right]\left[\prod_{\substack{h \notin b, \alpha_{h}=4}} M^{-i_{h}} \prod_{\substack{\bar{h} \notin b, \alpha_{\bar{h}}=4}} M^{-i_{\bar{h}}}\right]} \\
& {\left[\prod_{\substack{h \notin b, \alpha_{h}=5}} M^{-i_{v_{h}}} n_{d}\left(\Delta_{i_{v_{h}}}\right) \prod_{\substack{\bar{h} \notin b, \bar{\alpha}_{\bar{h}}=5}} M^{-i_{v_{\bar{h}}}} n_{d}\left(\Delta_{i_{\bar{h}}}\right)\right]}
\end{aligned}
$$

where

$$
\left|C_{\Delta_{l} \bar{\Delta}_{l}}^{j k_{l}}\left(x_{l}, \bar{x}_{l},\left\{w_{l^{\prime}}^{\prime}\right\},\left\{w_{l^{\prime}}^{\prime \prime}\right\}\right)\right| \leq\left|C^{j k_{l}}\left(x_{l}, \bar{x}_{l}\right)\right| .
$$

To get the factor $\prod_{v \in V_{d} \cup V_{b}}\left[|\ln T|^{3 / 4}\right] \prod_{v \notin V_{d} \cup V_{b}}|\ln T|$ in this bound we collected the factors $j_{M}^{1 / 4}$, which are bounded by $|\ln T|^{1 / 4}$ (II.32), and we used the fact that a vertex $v \in V_{d} \cup V_{b}$ either is in $V_{d}$, hence has a field or antifield of type 5 hooked to it, which has no $j_{M}^{1 / 4}$ factor, or is in $V_{b}-V_{d}$, hence has at least a field or antifield in $b$ which does not appear in the products of (IV.176).

The integrals over the weakening factors $w_{l}$ and $w_{l}^{\prime \prime}$ have been bounded by one, but the ones over $w_{l}^{\prime}$ are kept preciously since they are used below. Now we observe that

$$
\sum_{\substack{\left\{r_{h}\right\},\left\{r_{\bar{h}}\right\} \\\left\{s_{h}\right\},\left\{s_{\bar{h}}\right\}}}\left[\prod_{\{h \notin b\}}\left[2^{-\frac{\left|r_{h}\right|}{8}-\frac{\left|s_{h}\right|}{4}}\right] \prod_{\{\bar{h} \notin b\}}\left[2^{-\frac{\left|r_{\bar{h}}\right|}{8}-\frac{\left|s_{\bar{h}}\right|}{4}}\right]\right] \leq C^{n}
$$

The logarithms are bounded using the relation $|\ln T||\lambda| \leq K$. Hence we can write (since we assumed $K \leq 1$ )

$$
\prod_{v \in V_{d} \cup V_{b}}|\lambda||\ln T|^{3 / 4} \prod_{v \notin V_{d} \cup V_{b}}|\lambda||\ln T| \leq K^{\left|\bar{V}_{d} \backslash V_{b}\right|} \prod_{v \in V_{d} \cup V_{b}}|\lambda|^{\frac{1}{4}}
$$


The $n_{\Delta}$ and $n(\Delta)$ factors coming from the Hadamard bound can be estimated using Stirling's formula as follows:

$$
\begin{aligned}
& \prod_{\substack{h \notin b \\
h \notin b \\
\alpha_{h}=1}} n_{\Delta_{h}}^{\frac{1}{4}} \prod_{\substack{\bar{h} \notin b, \alpha_{\bar{h}}=1}} n_{\Delta_{\bar{h}}}^{\frac{1}{4}} \leq \prod_{\Delta \in B_{S}} n_{\Delta}^{n_{\Delta}} \leq \prod_{\Delta \in B_{S}} n_{\Delta} ! e^{n_{\Delta}}=e^{n} \prod_{\Delta \in B_{S}} n_{\Delta} ! \\
& \prod_{\substack{h \notin b, \alpha_{h}=5}} n_{d}\left(\Delta_{i_{v_{h}}}\right) \prod_{\substack{\bar{h} \notin b, \alpha \bar{h}=5}} n_{d}\left(\Delta_{i_{v_{\bar{h}}}}\right) \leq \prod_{\Delta \in Y} n_{d}(\Delta)^{n_{d}(\Delta)} \leq e^{n} \prod_{\Delta \in Y} n_{d}(\Delta) !
\end{aligned}
$$

Inserting all these results and absorbing all constants except $K$ in the global factor $C^{n}$ we have

$$
\begin{aligned}
& \sum_{\substack{Y \\
0 \in Y}}\left|A_{c}(Y)\right| L^{|Y|} \leq \sum_{M_{Y}} \sum_{S} \sum_{V L} L^{|Y|} \sum_{B_{S}} \sum_{\left\{x_{\Delta}\right\}^{c}} \sum_{n=0}^{\infty} \frac{\left(C M^{\frac{16}{3}} M^{4}\right)^{n}}{n !} K^{\left|\bar{V}_{d} \backslash V_{b}\right|} \sum_{V_{d}, \alpha_{V_{d}}} \\
& \sum_{a, b, R} \sum_{\left\{v_{i}\right\}_{l \in v L}} \sum_{n_{V_{d}} \sigma_{V_{d}} \rho_{V_{d}}} \sum_{\left\{n_{\Delta}\right\}_{\Delta \in B_{S}}} \sum_{\Delta_{V_{d}}^{c}}\left[\prod_{v \in V_{d}} \sum_{i_{v} \in I_{v}^{c}} \sum_{\Delta v}\right] \sum_{\left\{\mathcal{D}_{i v} \cap\right\}} \sum_{\left\{J_{h}^{a}\right\}\left\{J_{h}^{a}\right\}} \\
& \sum_{\left\{k_{h}\right\},\left\{k_{\bar{h}}\right\}} \sum_{\left\{\beta_{h}\right\}\left\{\beta_{\bar{h}}\right\}}\left[\prod_{v \in V_{d} \cup V_{b}}|\lambda|^{\frac{1}{4}}\right]\left[\prod_{v \notin V_{d} \cup V_{b}} M^{4 i_{\Delta_{v}}}\right]\left[\prod_{\Delta \in Y} n_{d}(\Delta) !\right] \\
& {\left[\prod_{\Delta \in B_{S}} n_{\Delta} !\right]\left[\prod_{\{h \notin b\}} M^{-\frac{k_{h}}{12}} \prod_{\{\bar{h} \notin b\}} M^{-\frac{k_{\bar{h}}}{12}}\right]\left[\prod_{\left\{\begin{array}{c}
h \notin b, \\
\alpha_{h}=1
\end{array}\right.} M^{-i_{\Delta_{h}}} \prod_{\substack{\bar{h} \notin b,\{\bar{h}=1 \\
\alpha \bar{h}}} M^{-i_{\Delta_{\bar{h}}}}\right]} \\
& {\left[\prod_{\substack{h \notin b, \alpha_{h}=2,3,5}} M^{-i_{v_{h}}} \prod_{\substack{\overline{\bar{h}} \notin b, \alpha_{\bar{h}}=2,3,5}} M^{-i_{v_{\bar{h}}}}\right]\left[\prod_{\substack{h \notin b, \alpha_{h}=4}} M^{-i_{h}} \prod_{\substack{\bar{h} \notin b \\
\alpha \bar{h}=4}} M^{-i_{\bar{h}}}\right]} \\
& \left(\prod_{j=m_{Y}}^{M_{Y}} \prod_{k=1}^{c_{j}} \sum_{T_{j k}}\right)\left(\prod_{v \in V_{d}} \int_{\Delta_{v}} d x_{v}\right)\left(\prod_{v \in V_{b} \backslash V_{d}} \int_{E x\left(\Delta_{v}\right)} d x_{v}\right)
\end{aligned}
$$




$$
\begin{aligned}
& {\left[\prod_{j=m_{Y}}^{M_{Y}} \prod_{k=1}^{c_{j}}\left[\prod_{l \in T_{j k}}\left|C_{\Delta_{l} \bar{\Delta}_{l}}^{j k_{l}}\left(x_{l}, \bar{x}_{l}\right)\right|\right] \delta_{k_{h_{l}} k_{\bar{h}_{l}}}\right]} \\
& {\left[\prod_{j=m_{Y}+1}^{M_{Y}}\left(\prod_{l \in v L_{j}} \int_{0}^{1} d w_{l}^{\prime}\right)\right]\left[\prod_{\substack{v \in V_{d} \\
c_{v} \neq \alpha_{v}}}^{l_{j}} \prod_{j=i_{v}} w_{y_{v}^{j}}^{\prime}\right]\left[\prod_{\substack{v \in V_{d} \\
c_{v}=\alpha_{v}}}^{\prod_{j=i_{v}}-1} w_{y_{v}^{j}}^{\prime}\right]}
\end{aligned}
$$

where we applied $f_{\Delta} \geq M^{-4}$ to bound every factor $f_{\Delta_{h}}^{-\frac{1}{4}}$ by $M$. As we have at most four fields of type 1 per vertex $v \in \bar{V}_{d}$ we obtain at most the factor $M^{4 n}$.

\section{IV.4 Extracting power counting}

In order to extract the power counting for $h$-links we define

$$
C_{\Delta_{l} \bar{\Delta}_{l}}^{j k_{l}}\left(x_{l}, \bar{x}_{l}\right)=M^{-j_{h}^{b}} M^{-j \bar{h}} M^{-\varepsilon k_{h}} M^{-\varepsilon k_{\bar{h}}} D_{\Delta_{l} \bar{\Delta}_{l}}^{j k_{l}}\left(x_{l}, \bar{x}_{l}\right)
$$

where $h$ and $\bar{h}$ are the field, antifield contracted to form the propagator and $\varepsilon$ is some small constant $0<\varepsilon<1$ that will be determined later. Remark that $j_{h}^{b}=j_{\bar{h}}^{b}=j, k_{h}=k_{\bar{h}}=k_{l}$ by construction. The factor $M^{-\varepsilon k_{h}}$ is necessary to sum over $k_{h}$ and extract a small factor per cube. The factor $M^{-j_{h}^{b}}$ corresponds to a kind of power counting for the field.

Now we can write

$$
\begin{aligned}
& \sum_{Y \in Y}\left|A_{c}(Y)\right| L^{|Y|} \leq \sum_{M_{Y}} \sum_{S} \sum_{V L} L^{|Y|} \sum_{B_{S}} \sum_{\left\{x_{\Delta}\right\}^{c}} \sum_{n=0}^{\infty} \frac{\left(C M^{\frac{28}{3}}\right)^{n}}{n !} K^{\left|\bar{V}_{d} \backslash V_{b}\right|} \sum_{V_{d}, \alpha_{V_{d}}} \\
& \sum_{a, b, R} \sum_{\left\{v_{l}\right\}_{l \in v L}} \sum_{n_{V_{d}} \sigma_{V_{d}} \rho_{V_{d}}} \sum_{\left\{n_{\Delta}\right\}_{\Delta \in B_{S}}} \sum_{\Delta_{\bar{V}_{d}}^{c}}\left[\prod_{v \in V_{d}} \sum_{i_{v} \in I_{v}^{c}} \sum_{\Delta_{v} \in \mathcal{D}_{i_{v}} \cap Y}\right] \sum_{\left\{J_{h}^{a}\right\},\left\{J_{\bar{h}}^{a}\right\}} \sum_{\left\{j_{h}^{b}\right\},\left\{j \bar{b}_{\bar{h}}\right\}} \\
& \sum_{\left\{k_{h}\right\},\left\{k_{\bar{h}}\right\}} \sum_{\left\{\beta_{h}\right\}\left\{\beta_{\bar{h}}\right\}}\left[\prod_{v \in V_{d} \cup V_{b}}|\lambda|^{\frac{1}{4}}\right]\left[\prod_{v \notin V_{d} \cup V_{b}} M^{4 i_{\Delta_{v}}}\right]\left[\prod_{\Delta \in Y} n_{d}(\Delta) !\right] \\
& {\left[\prod_{\Delta \in B_{S}} n_{\Delta} !\right]\left[\prod_{\{h \notin b\}} M^{-\frac{k_{h}}{12}} \prod_{\{\bar{h} \notin b\}} M^{-\frac{k_{\bar{h}}}{12}}\right]\left[\prod_{\{h \in b\}} M^{-\varepsilon k_{h}} \prod_{\{\bar{h} \in b\}} M^{-\varepsilon k_{\bar{h}}}\right]}
\end{aligned}
$$




$$
\begin{aligned}
& {\left[\prod_{\substack{h \notin b, \alpha_{h}=1,2,3,5}} M^{-i_{\Delta_{h}}} \prod_{\left\{\begin{array}{c}
\bar{h} \notin b, \\
\alpha_{\bar{h}}=1,2,3,5
\end{array}\right\}} M^{-i_{\Delta_{\bar{h}}}}\right]\left[\prod_{\substack{h \notin b \mid \alpha_{h}=4, \\
\text { or } h \in b}} M^{-i_{h}} \prod_{\left\{\begin{array}{c}
\bar{h} \notin b \mid \alpha_{\bar{h}}=4, \\
\text { or } \bar{h} \in b
\end{array}\right\}} M^{-i_{\bar{h}}}\right]} \\
& \left(\prod_{j=m_{Y}}^{M_{Y}} \prod_{k=1}^{c_{j}} \sum_{T_{j k}}\right)\left(\prod_{v \in V_{d} \cup V_{b}} \int_{\Omega_{v}} d x_{v}\right)\left[\prod_{j=m_{Y}}^{M_{Y}} \prod_{k=1}^{c_{j}}\left[\prod_{l \in T_{j k}}\left|D_{\Delta_{l} \bar{\Delta}_{l}}^{j k_{l}}\left(x_{l}, \bar{x}_{l}\right)\right|\right] \delta_{k_{h_{l}} k_{\bar{h}_{l}}}\right] \\
& {\left[\prod_{j=m_{Y}+1}^{M_{Y}}\left(\prod_{l \in v L_{j}} \int_{0}^{1} d w_{l}^{\prime}\right)\right]\left[\prod_{\substack{v \in V_{d} \\
c_{v} \neq \alpha_{v}}} \prod_{j=i_{v}}^{l_{v}} w_{y_{v}^{j}}^{\prime}\right]\left[\prod_{\substack{v \in V_{d} \\
c_{v}=\alpha_{v}}} \prod_{j=i_{v}}^{l_{v}-1} w_{y_{v}^{j}}^{\prime}\right]}
\end{aligned}
$$

where we defined $i_{h}=j_{h}^{b}$ if $h \in b$, and we defined $\Omega_{v}=\Delta_{i_{v}}$ if $v \in V_{d}$ and $\Omega_{v}=\operatorname{Ex}\left(\Delta_{v}\right)$ if $v \in V_{b} \cap \bar{V}_{d}$. We also defined $\Delta_{h}=\Delta_{v_{h}} \in B_{S}$ if $\alpha_{h}=1$ and $\Delta_{h}=\Delta_{i_{h}}$ if $\alpha_{h}=2,3,5$. Now for each $h$ with $i_{h}>i_{\Delta_{h}}$ we can write

$$
M^{-i_{h}}=M^{-i_{\Delta_{h}}} M^{-\left(i_{h}-i_{\Delta_{h}}\right)} .
$$

The same formulas hold for $\bar{h}$. The factor $M^{-i_{\Delta_{h}}}$ will be used to compensate the integration over $x_{v} \in \Delta_{v_{h}}$.

To extract power counting for a $v$-link associated to the vertex $v$ we extract a fraction $|\lambda|^{\frac{1}{8}}$ for each vertex in $V_{d}$ :

$$
\prod_{v \in V_{d}}|\lambda|^{\frac{1}{4}}=\prod_{v \in V_{d}}|\lambda|^{\frac{1}{8}}|\lambda|^{\frac{1}{8}}
$$

Now, for each $y_{k}^{j}$ connected to its ancestor by a $f$-link, there are 6 external fields. One of these may be the field $h_{\text {root }}$. For this field we keep the vertical decay $M^{-\left(j_{h}^{b}-i_{\Delta_{h}}\right)}$ untouched, in order to perform later the sum over the tree structure.

The vertical decay for the remaining five external fields, together with the factors $\lambda^{\frac{1}{8}}$ are necessary for several purposes:

- to ensure a factor $M^{-4}$ to sum the root cube for any $y_{k}^{j}$ inside a cube at scale $j+1$;

- to sum over $J_{h}^{a}$ and $j_{h}^{b}$;

- to extract one small factor per cube; 
- to sum over the tree structure.

Therefore we write the vertical decay for each of the five fields as follows:

$$
M^{-\left(i_{h}-i_{\Delta_{h}}\right)}=M^{-\frac{\varepsilon^{\prime}}{2}\left(i_{h}-i_{\Delta_{h}}\right)} M^{-\frac{\varepsilon^{\prime}}{2}\left(i_{h}-i_{\Delta_{h}}\right)} M^{-\left(1-\varepsilon^{\prime}\right)\left(i_{h}-i_{\Delta_{h}}\right)}
$$

where $0<\varepsilon^{\prime}<1$ is some small constant that will be chosen later. One of the two fractions $\varepsilon^{\prime} / 2$ is necessary to sum over $J_{h}^{a}$ and $j_{h}^{b}$, and to extract one small factor per cube. The other fraction will be used to reconstruct some vertical decay in order to sum over the tree. Now we call $G F$ the set of subpolymers $y_{k}^{j}$ connected to their ancestor by a $f$-link, and $G V$ the set of subpolymers $y_{k}^{j}$ connected to their ancestor by a $v$-link. Therefore we can write

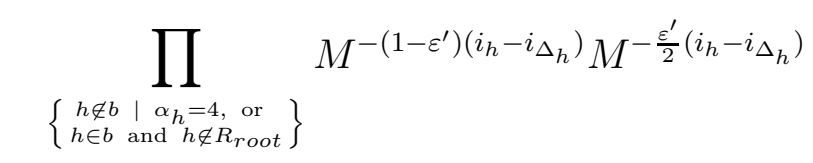

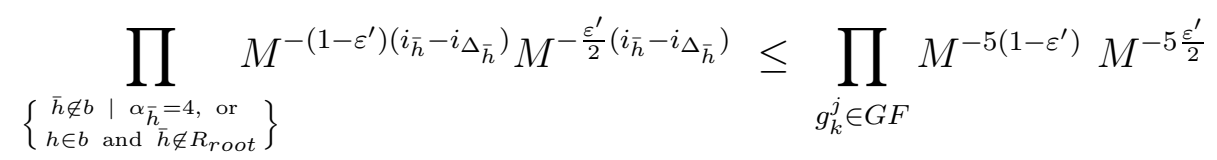

where we applied the equation

$$
M^{-\left(1-\varepsilon^{\prime}\right)\left(i_{h}-i_{\Delta_{h}}\right)}=\prod_{j=i_{h}}^{i_{\Delta_{h}}-1} M^{-\left(1-\varepsilon^{\prime}\right)[j-(j-1)]}
$$

On the other hand for subpolymers with $v$-links we write

$$
\prod_{v \in V_{d}}|\lambda|^{\frac{1}{8}}=\prod_{y_{k}^{j} \in G V}|\lambda|^{\frac{1}{8}} \leq\left[\prod_{y_{k}^{j} \in G V} M^{-5\left(1-\varepsilon^{\prime}\right)}\right]\left[\prod_{v \in V_{d}}|\lambda|^{\frac{\varepsilon^{\prime}}{8}}\right]
$$

where from now on we assume

$$
|\lambda|^{\frac{1}{8}} \leq M^{-5}
$$

Finally we observe that for each $h \in R_{\text {root }}$ we can reconstruct a fraction of the vertical decay $j_{h}^{b}-i_{\Delta_{h}}$. This is possible because any cube $\Delta$ in the set

$$
A_{h}=:\left\{\Delta \mid j_{h}^{b}>i_{\Delta} \geq i_{\Delta_{h}} \text { and } \Delta_{h} \subseteq \Delta\right\}
$$


must be $\Delta_{\text {root }}^{0}$ for some connected component at scale $i_{\Delta}$, with $\Delta_{h} \subseteq \Delta$, and we can extract a fraction $|\lambda|^{\frac{\varepsilon^{\prime}}{16}}$ or $M^{-5 \frac{\varepsilon^{\prime}}{2}}$ of its vertical decay. Remark that no field $h^{\prime} \neq h \in R_{\text {root }}$ can hook to any cube in $A_{h}$, because they are all cubes of type $\Delta_{\text {root }}^{0}$, therefore $A_{h} \cap A_{h^{\prime}}=\emptyset$ for any $h^{\prime} \neq h \in R_{\text {root }}$. This means that the same $\Delta$ is never used for more than one $h \in R_{\text {root }}$. Therefore we can write

$$
\left[\prod_{v \in V_{d}}|\lambda|^{\frac{\varepsilon^{\prime}}{8}}\right]\left[\prod_{y_{k}^{j} \in G F} M^{-5 \frac{\varepsilon^{\prime}}{2}}\right] \leq\left[\prod_{h \in R_{\text {root }}} M^{-5 \frac{\varepsilon^{\prime}}{2}\left(i_{h}-i_{\Delta_{h}}\right)}\right]\left[\prod_{v \in V_{d}}|\lambda|^{\frac{\varepsilon^{\prime}}{16}}\right]
$$

One of this fractions can be used to sum over $j_{h}^{b}$, the others will be used to sum over the tree. Inserting all these bounds we have

$$
\begin{aligned}
& \sum_{\substack{Y \\
0 \in Y}}\left|A_{c}(Y)\right| L^{|Y|} \leq \sum_{M_{Y}} \sum_{S} \sum_{V L} L^{|Y|} \sum_{B_{S}} \sum_{\left\{x_{\Delta}\right\}^{c}} \sum_{n=0}^{\infty} \frac{\left(C M^{\frac{28}{3}}\right)^{n}}{n !} K^{\left|\bar{V}_{d} \backslash V_{b}\right|} \\
& \sum_{V_{d}, \alpha_{V_{d}}} \sum_{a, b, R} \sum_{\left\{v_{l}\right\}_{l \in v L}} \sum_{n_{V_{d}} \sigma_{V_{d}} \rho_{V_{d}}} \sum_{\left\{n_{\Delta}\right\}_{\Delta \in B_{S}}} \sum_{\Delta_{V_{d}}^{c}}\left[\prod_{v \in V_{d}} \sum_{i_{v} \in I_{v}^{c}} \sum_{\Delta, \mathcal{D}_{i_{v}} \cap Y}\right] \sum_{\left\{J_{h}^{a}\right\},\left\{J_{h}^{a}\right\}} \\
& \sum_{\left\{j_{h}^{b}\right\},\left\{j_{\bar{h}}^{b}\right\}} \sum_{\left\{k_{h}\right\},\left\{k_{\bar{h}}\right\}} \sum_{\left\{\beta_{h}\right\}\left\{\beta_{\bar{h}}\right\}}\left[\prod_{v \in V_{d} \cup V_{b}}|\lambda|^{\frac{1}{16}}\right]\left[\prod_{v \in V_{d} \cup V_{b}}|\lambda|^{\frac{1}{16}}\right] \\
& {\left[\prod_{v \in V_{d} \cup V_{b}} M^{-4 i_{\Delta v}}\right]\left[\prod_{\Delta \in Y} n_{d}(\Delta) !\right]\left[\prod_{\Delta \in B_{S}} n_{\Delta} !\right]\left[\prod_{\{h \notin b\}} M^{-\frac{k_{h}}{12}} \prod_{\{\bar{h} \notin b\}} M^{-\frac{k_{\bar{h}}}{12}}\right]} \\
& {\left[\prod_{\{h \in b\}} M^{-\varepsilon k_{h}} \prod_{\{\bar{h} \in b\}} M^{-\varepsilon k_{\bar{h}}}\right]\left[\prod_{\left\{h \in R_{\text {root }}\right\}} M^{-\left(i_{h}-i_{\Delta_{h}}\right)} \prod_{\left\{\bar{h} \in R_{\text {root }}\right\}} M^{-\left(i_{\bar{h}}-i_{\Delta_{\bar{h}}}\right)}\right]}
\end{aligned}
$$

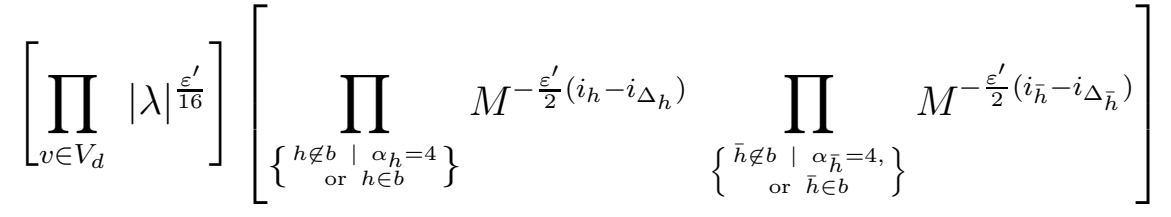

$$
\begin{aligned}
& {\left[\prod_{\left\{h \in R_{\text {root }}\right\}} M^{-4 \frac{\varepsilon^{\prime}}{2}\left(i_{h}-i_{\Delta_{h}}\right)} \prod_{\left\{\bar{h} \in R_{\text {root }}\right\}} M^{-4 \frac{\varepsilon^{\prime}}{2}\left(i_{\bar{h}}-i_{\bar{h}_{\bar{h}}}\right)}\right]\left[\prod_{j=m_{Y}}^{M_{Y}-1} \prod_{k=1}^{c_{j}} M^{-5\left(1-\varepsilon^{\prime}\right)}\right]}
\end{aligned}
$$




$$
\begin{aligned}
& \left(\prod_{j=m_{Y}}^{M_{Y}} \prod_{k=1}^{c_{j}} \sum_{T_{j k}}\right)\left(\prod_{v \in V_{d} \cup V_{b}} \int_{\Omega_{v}} d x_{v}\right)\left[\prod_{j=m_{Y}}^{M_{Y}} \prod_{k=1}^{c_{j}}\left[\prod_{l \in T_{j k}}\left|D_{\Delta_{l} \bar{\Delta}_{l}}^{j k_{l}}\left(x_{l}, \bar{x}_{l}\right)\right|\right] \delta_{\left.k_{h_{l}} k_{\bar{h}_{l}}\right]}\right] \\
& {\left[\prod_{j=m_{Y}+1}^{M_{Y}}\left(\prod_{l \in v L_{j}} \int_{0}^{1} d w_{l}^{\prime}\right)\right]\left[\prod_{\substack{v \in V_{d} \\
c_{v} \neq \alpha_{v}}} \prod_{j=i_{v}}^{l_{v}} w_{y_{v}^{j}}^{\prime}\right]\left[\prod_{\substack{v \in V_{d} \\
c_{v}=\alpha_{v}}} \prod_{j=i_{v}}^{l_{v}-1} w_{y_{v}^{j}}^{\prime}\right]}
\end{aligned}
$$

where we applied

$$
\prod_{v \notin V_{d} \cup V_{b}} M^{4 i_{\Delta_{v}}} \prod_{v \notin V_{d} \cup V_{b}} M^{-4 i_{\Delta_{v}}}=1
$$

and

$$
\prod_{y_{k}^{j} \in G V} M^{-5\left(1-\varepsilon^{\prime}\right)} \prod_{y_{k}^{j} \in G F} M^{-5\left(1-\varepsilon^{\prime}\right)}=\left[\prod_{j=m_{Y}}^{M_{Y}-1} \prod_{k=1}^{c_{j}} M^{-5\left(1-\varepsilon^{\prime}\right)}\right]
$$

where we have extracted from $|\lambda|^{\frac{1}{8}}$ a fraction $|\lambda|^{\frac{1}{16}}$ that will be used to extract a small factor per cube. The factors $M^{-5\left(1-\varepsilon^{\prime}\right)}$ will be used to sum over the cube positions and to perform the last sum over $S$. Remember that $\Delta_{v}$ is a cube in $B_{S}$ if $v \in V_{b} \cap \bar{V}_{d}$, but if $v \in V_{d}$, the localization cube $\Delta_{i_{v}}$ of $v$ may not be a summit cube.

\section{IV.5 Extracting a small factor per cube}

Now, before bounding the polymer structure, we must extract a small factor $g$ for each cube, in order to obtain a factor $g^{|Y|}$.

First we still need to extract some fractions of vertical decay. Actually, we will need also a fraction of the $k$ decay for tree lines. Therefore we write

$$
M^{-\varepsilon k_{h}}=M^{-\frac{\varepsilon}{2} k_{h}} M^{-\frac{\varepsilon}{2} k_{h}}
$$

for each $h, \bar{h} \in b$. One fraction will be used to sum over $k_{h}$, and the remaining fraction is used to extract a small factor per cube. Finally we need to extract a fraction $\varepsilon^{\prime} / 4$ of the vertical decay $M^{-\frac{\varepsilon^{\prime}}{2}\left(i_{h}-i_{\Delta_{h}}\right)}$ for each $h, \bar{h} \notin b$ with $\alpha_{h}=4$, and for each $h, \bar{h} \in b$. One fraction will be used to sum over $J^{a}$ and $j^{b}$. The 
remaining fraction is bounded by

$$
\left[\prod_{\left\{\begin{array}{c}
h \notin b \mid \alpha_{h}=4 \\
\text { or } h \in b
\end{array}\right\}} M^{-\frac{\varepsilon^{\prime}}{4}\left(i_{h}-i_{\Delta_{h}}\right)} \prod_{\substack{\bar{h} \notin b \mid \alpha_{\bar{h}}=4, \\
\text { or } \bar{h} \in b}} M^{-\frac{\varepsilon^{\prime}}{4}\left(i_{\bar{h}}-i_{\Delta_{\bar{h}}}\right)}\right]\left[\prod_{v \in V_{d}}|\lambda|^{\frac{\varepsilon^{\prime}}{16}}\right] \leq\left[\prod_{j=m_{Y}}^{M_{Y}-1} \prod_{k=1}^{c_{j}} M^{-5 \frac{\varepsilon^{\prime}}{4}}\right] .
$$

Now we can prove the following lemma.

Lemma. One can extract from (IV.194) at least one small factor $g<1$ for each cube in $Y$, where $g$ is defined by

$$
g=\max \left[|\lambda|^{\frac{1}{32}}, M^{-5 \frac{\varepsilon^{\prime}}{4 d}}, M^{-\frac{\varepsilon}{2 d}}\right]
$$

where $d=3^{4}=81$ is the number of nearest neighbors for each cube (including itself).

Proof. We will proof the following inequality

$$
\left[\prod_{v \in V_{d} \cup V_{b}}|\lambda|^{\frac{1}{16}}\right]\left[\prod_{j=m_{Y}}^{M_{Y}-1} \prod_{k=1}^{c_{j}} M^{-5 \frac{\varepsilon^{\prime}}{4}}\right]\left[\prod_{h \in b} M^{-\frac{\varepsilon}{2} k_{h}} \prod_{\bar{h} \in b} M^{-\frac{\varepsilon}{2} k_{\bar{h}}}\right] \leq g^{|Y|}
$$

which is enough to prove the lemma.

First we make some remarks.

1) For all extremal summit cube $\Delta \in Y(\operatorname{Ex}(\Delta)=\Delta)$, there must be at least one vertex $v \in V_{d} \cup V_{b}$ with $\Delta_{v}=\Delta$, as this cube must be connected to the polymer by a horizontal or vertical link. For this vertex we have a factor $|\lambda|^{\frac{1}{16}} \leq g^{2}$. Therefore we a factor $g^{2}$ for each $\Delta \in Y$ with $\operatorname{Ex}(\Delta)=\Delta$.

2) For all $\Delta \in Y$ such that $\Delta=\Delta_{\text {root }}^{0}$ for some connected subpolymer $y_{k}^{j}$, there is a vertical link connecting $\Delta$ to its ancestor and we have a fraction of the vertical decay $M^{-5 \frac{\varepsilon^{\prime}}{4}} \leq g^{d}$.

3) For each tree line $C^{j k}$ connecting some $\Delta, \Delta^{\prime} \in \mathcal{D}_{j}$, we can write the vertical decay $M^{-\frac{\varepsilon}{2} k}$ for the corresponding $h$ and $\bar{h}\left(k_{h}=k_{\bar{h}}=k\right)$ as

$$
M^{-\frac{\varepsilon}{2} k} M^{-\frac{\varepsilon}{2} k}=\prod_{j^{\prime}=j}^{j+k-1} M^{-\frac{\varepsilon}{2}} M^{-\frac{\varepsilon}{2}}
$$


Therefore for all $\Delta^{\prime \prime} \in \mathcal{D}_{j^{\prime}}$ with $j \leq j^{\prime} \leq j+k-1$ such that $\Delta \subseteq \Delta^{\prime \prime}$ or $\Delta^{\prime} \subseteq \Delta^{\prime \prime}$ we have a factor $M^{-\frac{\varepsilon}{2}} \leq g^{\bar{d}}$.

With these remarks we can now prove (IV.200) by induction. Actually we will prove that, if at the scale $j$ we have a factor $g^{2}$ for any $\Delta \in \mathcal{D}_{j} \cap Y$ then we can rewrite this factors in such a way to have a factor $g$ for any $\Delta \in \mathcal{D}_{j} \cap Y$ and a factor $g^{2} \forall \Delta \in \mathcal{D}_{j+1} \cap Y$.

Inductive hypothesis: At scale $j$ we have a factor $g^{2}$ for any $\Delta \in \mathcal{D}_{j} \cap Y$.

This is certainly true for the highest scale $m_{Y}$, because at this scale all cubes are extremal summit cubes therefore by remark 1) they have a factor $g^{2}$.

Proof of the induction. Now we must prove that, given a factor $g^{2}$ for any $\Delta \in \mathcal{D}_{j} \cap Y$, we have a factor $g$ for any $\Delta \in \mathcal{D}_{j} \cap Y$ and a factor $g^{2}$ for any $\Delta \in \mathcal{D}_{j+1} \cap Y$.

We consider a connected component $y_{k}^{j+1}$. This is made from a set of generalized cubes connected by a tree. Let us consider one particular generalized cube $\tilde{\Delta}$ which is made of cubes of scale $j+1$ connected by links of higher scales. Now we consider each cube in $\tilde{\Delta}$. For each such $\Delta$ we denote by $s_{\Delta}$ the number of cubes above that is $s_{\Delta}=\left\{\Delta^{\prime} \in \mathcal{D}_{j} \cap Y \mid \Delta^{\prime} \subset \Delta\right\}$ We distinguish three situations.

a) If $\left|s_{\Delta}\right|=0$ then we are in the special case $\operatorname{Ex}(\Delta)=\Delta$ therefore the extremal summit cube $\Delta$ has a factor $g^{2}$.

b) If $\left|s_{\Delta}\right| \geq 2$ then we have

$$
g^{2\left|s_{\Delta}\right|}=g^{\left|s_{\Delta}\right|} g^{\left|s_{\Delta}\right|} \leq g^{\left|s_{\Delta}\right|} g^{2}
$$

therefore we can keep a factor $g$ for each $\Delta^{\prime} \in s_{\Delta}$ and we have a factor $g^{2}$ for $\Delta$.

c) The case $\left|s_{\Delta}\right|=1$ is the most difficult one. We call the unique element of $s_{\Delta} \Delta^{\prime}$. Again we distinguish three cases:

- there is no tree line of any scale connecting $\Delta^{\prime}$ to some other $\Delta^{\prime \prime} \in \mathcal{D}_{j}$ (see Fig.10 a). Therefore $\Delta=\tilde{\Delta}$ and $\Delta^{\prime}$ must be $\Delta_{\text {root }}^{0}$ for some connected component at scale $j$, therefore there is a vertical link connecting 

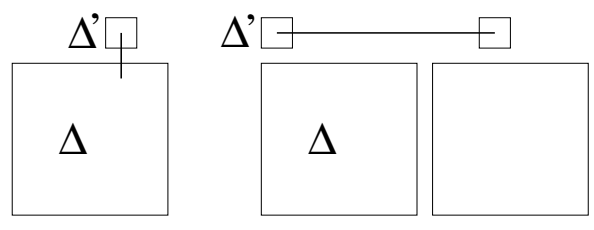

b

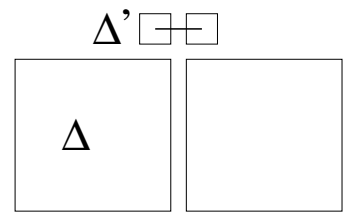

$\mathrm{c}$

Figure 10: Three possible cases for $\left|s_{\Delta}\right|=1$.

$\Delta^{\prime}$ to $\Delta$, and, by 3 ) we have a factor $g^{d}$. Hence we can write

$$
g^{2} g^{d}=g g^{d+1} \leq g g^{2}
$$

and we can keep a factor $g$ for $\Delta^{\prime}$ and assign a factor $g^{2}$ to $\Delta$.

- there is at least one tree line $C^{j^{\prime} k}$ at some scale $j^{\prime} \leq j$ connecting $\Delta^{\prime}$ to some $\Delta^{\prime \prime} \subset \Delta_{1}\left(\Delta_{1} \in \tilde{\Delta}\right.$ ) and $\Delta^{\prime}$ is not nearest neighbor of $\Delta^{\prime \prime}$ (see Fig.10 b).

Then, $\left|t_{\Delta^{\prime}}-t_{\Delta^{\prime \prime}}\right| \geq M^{j+1}$ (in the space directions they must always be nearest neighbors) and the propagator must have $j^{\prime}+k \geq j+1$. Therefore as $j^{\prime} \leq j k$ cannot be zero and by remark 3 ) we can associate to $\Delta^{\prime}$ a factor $g^{d}$ in addition to $g^{2}$. Hence we can write

$$
g^{2} g^{d}=g g^{d+1} \leq g g^{2}
$$

and we can keep a factor $g$ for $\Delta^{\prime}$ and assign a factor $g^{2}$ to $\Delta$.

- there is at least one tree line $C^{j^{\prime} k}$ at some scale $j^{\prime} \leq j$ connecting $\Delta^{\prime}$ to some $\Delta^{\prime \prime} \subset \Delta_{1}\left(\Delta_{1} \in \tilde{\Delta}\right.$ ) and $\Delta^{\prime}$ is nearest neighbor of $\Delta^{\prime \prime}$ (see Fig.10 c).

In this case $j^{\prime}+k \geq j$ and no factor can be extracted from the $k$ decay. Remark that, if $\Delta^{\prime}=\Delta_{\text {root }}^{0}$ for some connected component at scale $j$, then there is a vertical link and everything works as in the case of Fig 10 a). On the other hand, if $\Delta^{\prime} \neq \Delta_{\text {root }}^{0}$, there is still a vertical link connecting $\Delta^{\prime}$ to $\Delta$ but it does not have any vertical decay associated. In this case we have to distinguish three possible situations: 
a' ) there is no other tree line connecting $\Delta^{\prime}$ or $\Delta^{\prime \prime}$ to some other cube in $\mathcal{D}_{j}$. Therefore $\Delta^{\prime \prime}$ must be $\Delta_{\text {root }}^{0}$ for some connected component at scale $j$ and the corresponding vertical link has a vertical decay associated. Hence we have a factor $g$ in addition to $g^{2}$ for each cube nearest neighbor (nn) of $\Delta^{\prime \prime}$, hence for each of them we can keep one factor $g$ and give the remaining $g^{2}$ to its ancestor.

b' ) there is a tree line connecting $\Delta^{\prime \prime}$ to some cube which is not nn of $\Delta^{\prime \prime}$. Then we have some $k$ vertical decay from the tree propagator, and we can assign a factor $g$ in addition to $g^{2}$ for each cube nn of $\Delta^{\prime \prime}$. Therefore, as in a', we have a factor $g$ in addition to $g^{2}$ for each cube nn of $\Delta^{\prime \prime}$, hence for each of them we can keep one factor $g$ and give the remaining $g^{2}$ to its ancestor.

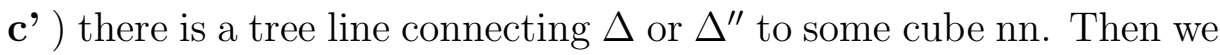
test case a' and b' again, and we go on until a' or b' (see Fig[1] $\mathrm{a}, \mathrm{b})$ is satisfied, or until the chain of nn cubes at scale $j$ arrives to a cube at scale $j+1$ that is not nn of $\Delta$. In this last case (see Fig11 c) we must have at least $M$ of such cubes, therefore we can write

$$
\left(g^{2}\right)^{M} \leq g^{M}\left(g^{2}\right)^{d}
$$

which means that we keep one factor $g$ for each cube at scale $j$ and we give a factor $g^{2}$ to each nn of $\Delta$ at scale $j+1$. This is true if $M$ satisfies:

$$
M \geq 2 d .
$$

\section{IV.6 Bounding the tree choice}

Construction of $T_{j k} \quad$ Before summing over the trees we must see how the tree is built. In the connected component $y_{j k}$, for each $\tilde{\Delta} \neq \tilde{\Delta}_{\text {root }}$ we have one $h \in R_{\text {root }}$ and $d_{\tilde{\Delta}}$ fields in $l_{b}(\tilde{\Delta})$ (defined in sec. III.3). For $\tilde{\Delta}_{\text {root }}$ we have no $h \in R_{\text {root }}$ but we still have $d_{\tilde{\Delta}}$ fields in $l_{b}(\tilde{\Delta})$. Each $h \in l_{b}(\tilde{\Delta})$ can contract only with a $h \in R_{\text {root }}$ in some $\tilde{\Delta} \neq \tilde{\Delta}^{\prime}$. As there is only one field $h \in b\left(\tilde{\Delta}^{\prime}\right)$ we only have to choose $\tilde{\Delta}^{\prime}$. This last sum is performed using the decay of the tree line as we will prove below. 

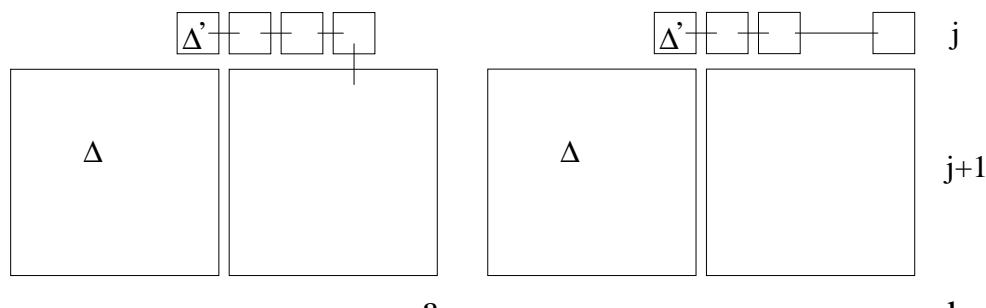

a

b

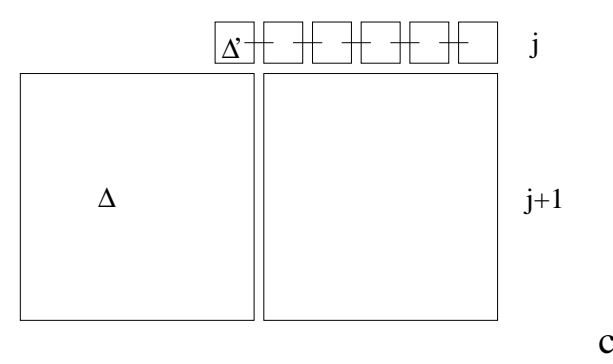

Figure 11: Three possible situations when extracting a small factor $g$

Therefore for each $h \in l_{b}(\tilde{\Delta})$ we have to perform the following sum

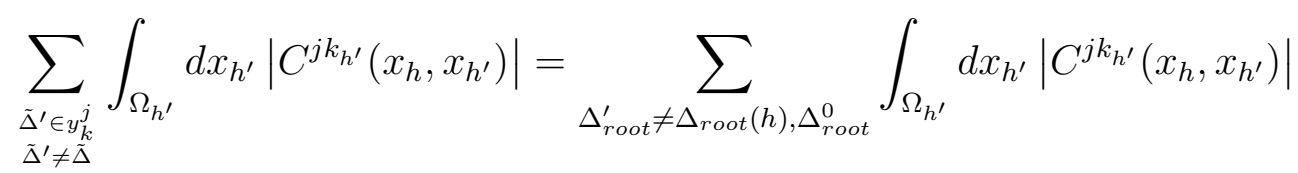

where $h^{\prime}$ is the unique field in $R_{\text {root }}$ hooked to $\tilde{\Delta}^{\prime}, \Omega_{h^{\prime}}$ is the localization volume $\Omega_{v_{h^{\prime}}}$ of the vertex to which $h^{\prime}$ is hooked, $\Delta_{\text {root }}^{\prime}$ is the corresponding cube in $\tilde{\Delta}^{\prime}$ and $\Delta_{h^{\prime}} \subseteq \Delta_{\text {root }}^{\prime}$ is the localization cube for $h^{\prime}$. Finally we denoted by $\Delta_{\text {root }}(h)$ the cube $\Delta_{\text {root }}$ for the generalized cube $\tilde{\Delta}$ where $h$ is hooked (this contraction is not possible as it would generate a loop). Remark that the condition $\Delta_{\text {root }}^{\prime} \neq \Delta_{\text {root }}^{0}$ holds because this last cube does not contain any $h \in R_{\text {root }}$. The sum over the tree $T_{j k}$ is then bounded by

$$
\prod_{\substack{h \in b \backslash R_{\text {root }} \\ j_{h}^{b}=j, \text { and } \Delta_{h} \subseteq y_{k}^{j}}} \sum_{\substack{\Delta_{\text {root }}^{\prime} \neq \Delta_{\text {root }}(h), \Delta_{\text {root }}^{0} \\ \Omega_{h^{\prime}}}} \int_{x_{h^{\prime}}} \mid C^{j k_{h^{\prime}}\left(x_{h}, x_{h^{\prime}}\right) \mid .}
$$

Sum over the cube positions and $T_{j k}$ Now, for fixed $T_{j k}$, we have a multiscale tree structure. We want to sum over the cube positions following this tree from the leaves towards the root (which is the cube $\Delta_{\text {root }}^{0}$ at scale 


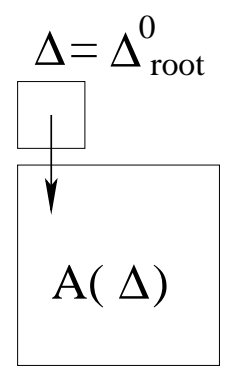

a

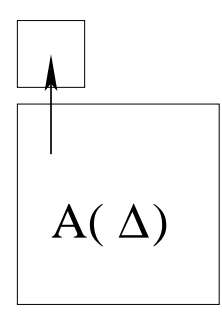

b

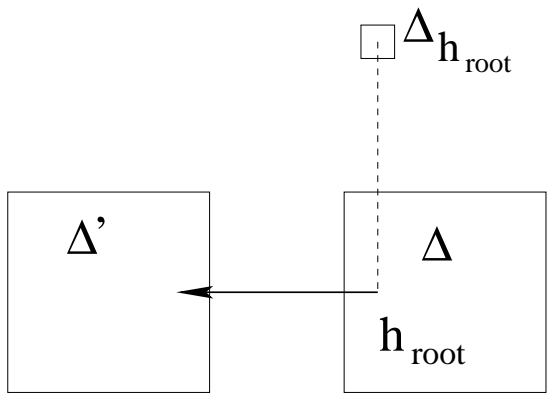

$\mathrm{c}$

Figure 12: Three types of oriented links

$M_{Y}$, which contain $x=0$ ). For this purpose we give a direction (represented by an arrow) to all links (vertical and horizontal).

- For any vertical link connecting some $\Delta_{\text {root }}^{0}$ to its ancestor we draw an arrow going from $\Delta_{\text {root }}^{0}$ down to its ancestor and we call it a down-link (see Fig.12a)

- For all other vertical links connecting some $\Delta$ to its ancestor we draw an arrow going from its ancestor up to $\Delta$ or and we call it a $u p-l i n k$ (see Fig.12b).

- For each horizontal link, that is made by the contraction of a field (antifield) in $R_{\text {root }}$ with an antifield ( field) in $b \backslash R_{\text {root }}$ we draw an arrow going from the field (antifield) in $R_{\text {root }}$ towards the antifield ( field) in $b \backslash R_{\text {root }}$ (see Fig.12] ).

Now we can perform the sums following the tree. We have three situations.

- If we have a down-link we have to sum over the choices for $\Delta$, for $\Delta^{\prime}=\mathcal{A}(\Delta)$ fixed. Remark that for each down-link we have the vertical decay $M^{-5\left(1-\varepsilon^{\prime}\right)}$. From this we first extract a fraction $M^{-5 \varepsilon^{\prime}}$ that will be used for the last sum. With the remaining $M^{-5\left(1-2 \varepsilon^{\prime}\right)}$ assuming 
$\varepsilon^{\prime} \leq 1 / 10$ we can write

$$
\sum_{\substack{\Delta \in \mathcal{D}_{j} \\ \Delta \subset \Delta^{\prime}, \Delta_{\Delta^{\prime}}=j+1}} M^{-5\left(1-2 \varepsilon^{\prime}\right)}=\frac{\left|\Delta^{\prime}\right|}{|\Delta|} M^{-5\left(1-2 \varepsilon^{\prime}\right)}=M^{4} M^{-5\left(1-2 \varepsilon^{\prime}\right)} \leq 1
$$

- If we have an up-link we have to sum over the choices for $\Delta^{\prime}=\mathcal{A}(\Delta)$ for $\Delta$ fixed. As there is only one $\Delta^{\prime}$ such that $\Delta^{\prime}=\mathcal{A}(\Delta)$ there is no sum at all.

- If we have an horizontal link the argument is more subtle and we explain it below.

Sum over horizontal links For some $h \in R_{\text {root }}$ we want to prove that

$$
M^{-\left(i_{h}-i_{\Delta_{h}}\right)} M^{-4 \frac{\varepsilon^{\prime}}{2}\left(i_{h}-i_{\Delta_{h}}\right)} \sum_{x_{\Delta}} \int_{\Omega_{h}} d x_{h}\left|D^{j k_{h}}\left(x_{h}, x_{h^{\prime}}\right)\right| \leq C M^{11 / 3} M^{4 i_{\Delta_{h}}}
$$

where $\Delta$ is the unique cube at scale $i_{h}=j_{h}^{b}=j$ with $\Delta_{h} \subseteq \Delta$ (see Fig.12 c). From now on we write $j$ instead of $i_{h}$. We recall that we defined for $k>0$ (see ([V.183) $)$

$$
\begin{aligned}
& \left|D^{j, k_{h}}\left(x_{h}, x_{h^{\prime}}\right)\right|=\left|C^{j, k_{h}}\left(x_{h}, x_{h^{\prime}}\right)\right| M^{2 j} M^{2 \varepsilon k_{h}} \\
& \leq C M^{8 / 3} M^{2 \varepsilon k_{h}} M^{-2 k_{h} / 3} \chi\left(\left|\vec{x}_{h}-\vec{x}_{h^{\prime}}\right| \leq M^{j-k_{h} / 3+1 / 3},\left|t_{h}-t_{h^{\prime}}\right| \leq M^{j+k}\right)
\end{aligned}
$$

and for $k=0$

$$
\begin{aligned}
\left|D^{j, 0}\left(x_{h}, x_{h^{\prime}}\right)\right| & =\left|C^{j, 0}\left(x_{h}, x_{h^{\prime}}\right)\right| M^{2 j} M^{2 \varepsilon k_{h}} \\
& \leq C M^{8 / 3} \chi\left(\left|\vec{x}_{h}-\vec{x}_{h^{\prime}}\right| \leq M^{j},\left|t_{h}-t_{h^{\prime}}\right| \leq M^{j}\right)
\end{aligned}
$$

The case $k=0$ is simple as

$$
\begin{aligned}
& \int_{\Omega_{h}} d x_{h} \chi\left(\left|\vec{x}_{h}-\vec{x}_{h^{\prime}}\right| \leq M^{j},\left|t_{h}-t_{h^{\prime}}\right| \leq M^{j}\right) \\
& \quad \leq M^{4 i_{\Delta_{h}}} \chi\left(\left|\vec{x}_{\Delta}-\vec{x}_{\Delta^{\prime}}\right| \leq M^{j},\left|t_{\Delta}-t_{\Delta^{\prime}}\right| \leq M^{j}\right)
\end{aligned}
$$

and

$$
\sum_{x_{\Delta}} \chi\left(\left|\vec{x}_{\Delta}-\vec{x}_{\Delta^{\prime}}\right| \leq M^{j},\left|t_{\Delta}-t_{\Delta^{\prime}}\right| \leq M^{j}\right) \leq d
$$


where $d$ is the number of nearest neighbors. Therefore

$$
M^{-\left(j-i_{\Delta_{h}}\right)} M^{-2 \varepsilon^{\prime}\left(j-i_{\Delta_{h}}\right)} \sum_{x_{\Delta}} \int_{\Omega_{h}} d x_{h}\left|D^{j k_{h}}\left(x_{h}, x_{h^{\prime}}\right)\right| \leq C M^{8 / 3} M^{4 i_{\Delta_{h}}}
$$

where the decay $M^{-\left(1+2 \varepsilon^{\prime}\right)\left(j-i_{\Delta_{h}}\right)}$ is just bounded by one.

The case $k>0$ is more difficult. Now the integral is given by

$$
\begin{aligned}
& \int_{\Omega_{h}} d x_{h} \chi\left(\left|\vec{x}_{h}-\vec{x}_{h^{\prime}}\right| \leq M^{j-k_{h} / 3+1 / 3},\left|t_{h}-t_{h^{\prime}}\right| \leq M^{j+k}\right) \\
& \leq M^{i_{\Delta_{h}}}\left(\min \left\{M^{i_{\Delta_{h}}}, M^{j-k_{h} / 3+1 / 3}\right\}\right)^{3} \chi\left(\left|\vec{x}_{\Delta}-\vec{x}_{\Delta^{\prime}}\right| \leq M^{j},\left|t_{\Delta}-t_{\Delta^{\prime}}\right| \leq M^{j+k}\right)
\end{aligned}
$$

and the sum over $x_{\Delta}$ gives

$$
\sum_{x_{\Delta}} \chi\left(\left|\vec{x}_{\Delta}-\vec{x}_{\Delta^{\prime}}\right| \leq M^{j},\left|t_{\Delta}-t_{\Delta^{\prime}}\right| \leq M^{j+k}\right) \leq d 2 M^{k}
$$

Now we have to distinguish two cases.

1. If we have

$$
i_{\Delta_{h}}<j-\frac{k_{h}}{3}+\frac{1}{3}
$$

(IV.210) is bounded by

$$
C M^{8 / 3} M^{4 i_{\Delta_{h}}} M^{k_{h}} M^{-\left(1+2 \varepsilon^{\prime}\right)\left(j-i_{\Delta_{h}}\right)} M^{-k_{h}(2 / 3-2 \varepsilon)}
$$

By (IV.218) we have

$$
M^{-\left(1+2 \varepsilon^{\prime}\right)\left(j-i_{\Delta_{h}}\right)} \leq M^{-\left(1+2 \varepsilon^{\prime}\right) k_{h} / 3} M^{\left(1+2 \varepsilon^{\prime}\right) / 3} .
$$

Inserting this bound in the equation above we obtain

$$
\left(C M^{8 / 3} M^{\left(1+2 \varepsilon^{\prime}\right) / 3}\right) M^{4 i_{\Delta_{h}}} M^{k_{h}\left(2 \varepsilon-2 \varepsilon^{\prime}\right)} \leq C M^{11 / 3} M^{4 i_{\Delta_{h}}}
$$

for $\varepsilon<\varepsilon^{\prime}$. 
2. On the other hand, if we have

$$
i_{\Delta_{h}} \geq j-k_{h} \frac{1}{3}+\frac{1}{3} \Rightarrow k_{h} \geq 3\left(j-i_{\Delta_{h}}\right)+1
$$

(IV.210) is bounded by

$$
C M^{8 / 3} M^{i_{\Delta_{h}}} M^{3\left(j-k_{h} / 3+1 / 3\right)} M^{k_{h}} M^{-\left(1+2 \varepsilon^{\prime}\right)\left(j-i_{\Delta_{h}}\right)} M^{-k_{h}(2 / 3-2 \varepsilon)}
$$

Now we can write

$$
M^{i_{\Delta_{h}}} M^{3\left(j-k_{h} / 3+1 / 3\right)} M^{k_{h}}=M M^{-k_{h}} M^{k_{h}} M^{4 i_{\Delta_{h}}} M^{3\left(j-i_{\Delta_{h}}\right)}
$$

and (IV.210) is bounded by

$$
\left(C M^{1+8 / 3}\right) M^{4 i_{\Delta_{h}}} M^{\left(2-2 \varepsilon^{\prime}\right)\left(j-i_{\Delta_{h}}\right)} M^{-k_{h}(2 / 3-2 \varepsilon)} \leq C M^{11 / 3} M^{4 i_{\Delta_{h}}}
$$

if we can prove that

$$
k_{h} \geq \frac{\left(1-\varepsilon^{\prime}\right)}{(1 / 3-\varepsilon)}\left(j-i_{\Delta_{h}}\right)
$$

This is true by (IV.222) if

$$
\frac{\left(1-\varepsilon^{\prime}\right)}{(1 / 3-\varepsilon)} \leq 3
$$

hence for $\varepsilon<\frac{\varepsilon^{\prime}}{3}$ which is consistent with the condition we find in the case $\mathbf{1}$.

With all these results we can now write

$$
\begin{aligned}
& \sum_{\left\{x_{\Delta}\right\}}\left[\prod_{\left\{h \in R_{\text {root }}\right\}} M^{-\left(i_{h}-i_{\Delta_{h}}\right)} \prod_{\left\{\bar{h} \in R_{\text {root }}\right\}} M^{-\left(i_{\bar{h}}-i_{\Delta_{\bar{h}}}\right)}\right]\left[\prod_{v \in V_{d} \cup V_{b}} M^{-4 i_{\Delta_{v}}}\right] \quad(\mathrm{IV} .228) \\
& {\left[\prod_{\left\{h \in R_{\text {root }}\right.} M^{-4 \frac{\varepsilon^{\prime}}{2}\left(i_{h}-i_{\Delta_{h}}\right)} \prod_{\left\{\bar{h} \in R_{\text {root }}\right\}} M^{-4 \frac{\varepsilon^{\prime}}{2}\left(i_{\bar{h}}-i_{\Delta_{\bar{h}}}\right)}\right]\left[\prod_{j=m_{Y}}^{M_{Y}-1} \prod_{k=1}^{c_{j}} M^{-5\left(1-2 \varepsilon^{\prime}\right)}\right]} \\
& {\left[\prod_{j=m_{Y}}^{M_{Y}} \prod_{k=1}^{c_{j}} \sum_{T_{j k}}\right]\left[\prod_{v \in V_{d} \cup V_{b}} \int_{\Omega_{v}} d x_{v}\right]\left[\prod_{j=m_{Y}}^{M_{Y}} \prod_{k=1}^{c_{j}}\left[\prod_{l \in T_{j k}}\left|D_{\Delta_{l} \bar{\Delta}_{l}}^{j k_{l}}\left(x_{l}, \bar{x}_{l}\right)\right|\right] \delta_{k_{h_{l}} k_{\bar{h}}}\right]} \\
& \leq C^{|Y|} M^{11 n / 3} \prod_{v \in V_{d} \cup V_{b}} M^{4 i_{\Delta_{v}}} M^{-4 i_{\Delta_{v}}} \leq C^{|Y|} M^{11 n / 3}
\end{aligned}
$$


where the constant $C^{|Y|}$ comes from ([V.210), and we applied

$$
\prod_{h \in R_{\text {root }}} M^{4 i_{\Delta_{h}}} \prod_{\bar{h} \in R_{\text {root }}} M^{4 i_{\Delta_{\bar{h}}}} \leq \prod_{v \in V_{d} \cup V_{b}} M^{4 i_{\Delta_{v}}}
$$

This is true because two $h_{\text {root }}$ cannot be hooked to the same vertex by construction.

\section{IV.7 Final bound}

Now we can perform all the remaining bounds, namely

$$
\begin{aligned}
& \sum_{\substack{Y \\
0 \in Y}}\left|A_{c}(Y)\right| L^{|Y|} \leq \sum_{M_{Y}} \sum_{S} \sum_{V L} L^{|Y|} \sum_{B_{S}} g^{|Y|} C^{|Y|} \sum_{n=0}^{\infty} C^{n} M^{13 n} \frac{1}{n !} \\
& |\lambda|^{\mid \frac{\left|V_{d} \cup V_{b}\right|}{16}} K^{\left|\bar{V}_{d} \backslash V_{b}\right|} \sum_{V_{d}, \alpha_{V_{d}}} \sum_{a, b, R} \sum_{\left\{v_{l}\right\}_{l \in v L}} \sum_{n_{V_{d}} \sigma_{V_{d}} \rho_{V_{d}}} \sum_{\left\{n_{\Delta}\right\}_{\Delta \in B_{S}}} \sum_{\Delta_{V_{d}}^{c}} \\
& {\left[\prod_{v \in V_{d}} \sum_{i_{v} \in I_{v}^{c}} \sum_{\Delta_{v} \in \mathcal{D}_{i_{v}} \cap Y}\right] \sum_{\left\{J_{h}^{a}\right\},\left\{J_{\bar{h}}^{a}\right\}} \sum_{\left\{j_{h}^{b}\right\},\left\{j_{\bar{h}}\right\}} \sum_{\left\{k_{h}\right\},\left\{k_{\bar{h}}\right\}} \sum_{\left\{\beta_{h}\right\}\left\{\beta_{\bar{h}}\right\}}} \\
& {\left[\prod_{\Delta \in Y} n_{d}(\Delta) !\right]\left[\prod_{\Delta \in B_{S}} n_{\Delta} !\right]\left[\prod_{\{h \notin b\}} M^{-\frac{k_{h}}{12}} \prod_{\{\bar{h} \notin b\}} M^{-\frac{k_{\bar{h}}}{12}}\right]\left[\prod_{\{h \in b\}} M^{-\frac{\varepsilon}{2} k_{h}}\right]} \\
& {\left[\prod_{\{\bar{h} \in b\}} M^{-\frac{\varepsilon}{2} k_{\bar{h}}}\right]\left[\prod_{\substack{\left\{\notin b \mid \alpha_{h}=4 \\
\text { or } h \in b\right.}} M^{-\frac{\varepsilon^{\prime}}{4}\left(i_{h}-i_{\Delta_{h}}\right)} \prod_{\substack{\bar{h} \notin b \mid \alpha_{\bar{h}}=4, \\
\text { or } \bar{h} \in b}} M^{-\frac{\varepsilon^{\prime}}{4}\left(i_{\bar{h}}-i_{\Delta_{\bar{h}}}\right)}\right]} \\
& {\left[\prod_{j=m_{Y}}^{M_{Y}-1} \prod_{k=1}^{c_{j}} M^{-5 \varepsilon^{\prime}}\right]\left[\prod_{j=m_{Y}+1}^{M_{Y}}\left(\prod_{l \in v L_{j}} \int_{0}^{1} d w_{l}^{\prime}\right)\right]\left[\prod_{\substack{v \in V_{d} \\
c_{v} \neq \alpha_{v}}} \prod_{j=i_{v}}^{l_{v}} w_{y_{v}^{j}}^{w^{\prime}}\right]\left[\prod_{\substack{v \in V_{d} \\
c_{v}=\alpha_{v}}} \prod_{j=i_{v}}^{l_{v}-1} w_{y_{v}^{\prime}}^{\prime}\right]}
\end{aligned}
$$

where $M^{-5 \varepsilon^{\prime}}$ is the factor we extracted form $M^{-5\left(1-\varepsilon^{\prime}\right)}$ before performing the sum over the tree choice. Now we can immediately bound the following sums.

- the sum over $\beta_{h}$ costs only a factor 5 per field, hence

$$
\sum_{\left\{\beta_{h}\right\}\left\{\beta_{\bar{h}}\right\}} 1 \leq 5^{4 n}
$$


- the sum over $k_{h}$ is performed using the vertical decay

$$
\sum_{\left\{k_{h}\right\},\left\{k_{\bar{h}}\right\}} \prod_{\{h \notin b\}} M^{-\frac{k_{h}}{12}} \prod_{\{\bar{h} \notin b\}} M^{-\frac{k_{\bar{h}}}{12}} \prod_{\{h \in b\}} M^{-\frac{\varepsilon}{2} k_{h}} \prod_{\{\bar{h} \in b\}} M^{-\frac{\varepsilon}{2} k_{\bar{h}}} \leq C^{n}
$$

- the sums over $J_{h}^{a}$ and $j_{h}^{b}$ are done using the vertical decay $M^{-\frac{\varepsilon^{\prime}}{4}\left(i_{h}-i_{\Delta_{h}}\right)}$ For $j_{h}^{b}$ we write

$$
\sum_{\left\{j_{h}^{b}\right\},\left\{j_{\bar{h}}^{b}\right\}} \prod_{\{h \in b\}} M^{-\frac{\varepsilon^{\prime}}{4}\left(j_{h}^{b}-i_{\Delta_{h}}\right)} \prod_{\{\bar{h} \in b\}} M^{-\frac{\varepsilon^{\prime}}{4}\left(j_{h}^{b}-i_{\Delta_{\bar{h}}}\right)} \leq C^{\left|V_{b}\right|}
$$

For $J_{h}^{a}$ we define $V_{d}^{\prime}$ as the set of vertices $v \in V_{d}$ that have some field (or antifield) $h \in a$. Then we can write

$$
\prod_{v \in V_{d}^{\prime}} \prod_{\left\{h_{v}^{c} \in a\right\}} \sum_{J_{h}^{a}} M^{-\frac{\varepsilon^{\prime}}{4}\left(i_{h}-i_{\Delta_{h}}\right)} \leq C^{n}
$$

where we applied $i_{\Delta_{h}}=i_{v}\left(\right.$ as $\left.v \in V_{d}\right)$ and

$$
\begin{aligned}
& \sum_{J_{h}^{a}} M^{-\frac{\varepsilon^{\prime}}{4}\left(i_{h}-i_{v}\right)}=\sum_{i_{h}>i_{v}} \sum_{p=0}^{i_{h}-i_{v}-1} \sum_{i_{v}<j_{1}<j_{2} \ldots<j_{p}<i_{h}} M^{-\frac{\varepsilon^{\prime}}{4}\left(i_{h}-i_{v}\right)} \\
& \leq \sum_{i_{h}} M^{-\frac{\varepsilon^{\prime}}{4}\left(i_{h}-i_{v}\right)} \sum_{p=0}^{i_{h}-i_{v}-1} 2^{\left(i_{h}-i_{v}\right)} \leq C
\end{aligned}
$$

and we used $\sum_{p=0}^{m} \sum_{0<j_{1}<j_{2} \ldots<j_{p}<m} 1 \leq 2^{m}$

\section{IV.7.1 Choice of $i_{v}$ and $\Delta_{i_{v}}$}

For each vertex $v_{l} \in V_{d}$ associated to the vertical link $l \in v L$ we can sum over the choices for $i_{v}$ and $\Delta_{i_{v}}$ using the weakening factors $w^{\prime}$. Actually these factors not only allow to choose $i_{v}$ and $\Delta_{i_{v}}$, but they also give a factor $1 / n_{d}(\Delta)$ ! for each $\Delta$, where we recall that $n_{d}(\Delta)$ is the number of vertices $v \in V_{d}$ localized in $\Delta$ (IV.120). This is proved in the following lemma. 
Lemma IV.7.1a The integrals over the weakening factors $w^{\prime}$ allow to choose $i_{v}$ and $\Delta_{i_{v}}$, and give a factor $1 / n_{d}(\Delta)$ ! for each $\Delta$, namely

$$
\begin{aligned}
& {\left[\prod_{v \in V_{d}} \sum_{i_{v} \in I_{v}^{c}} \sum_{\Delta_{i_{v}} \in \mathcal{D}_{i_{v}} \cap Y}\right]\left[\prod_{j=m_{Y}+1}^{M_{Y}}\left(\prod_{l \in v L_{j}} \int_{0}^{1} d w_{l}^{\prime}\right)\right]} \\
& {\left[\prod_{\substack{v \in V_{d} \\
c_{v} \neq \alpha_{v}}} \prod_{j=i_{v}}^{l_{v}} w_{y_{v}^{j}}^{\prime}\right]\left[\prod_{\substack{v \in V_{d} \\
c_{v}=\alpha_{v}}} \prod_{j=i_{v}}^{l_{v}-1} w_{y_{v}^{j}}^{\prime}\right] \leq C^{|Y|} \prod_{\Delta \in Y} \frac{1}{n_{d}(\Delta) !}(}
\end{aligned}
$$

Proof. We perform the sum following the structure of the rooted tree $S$. We call $T$ the tree obtained by $S$ taking away the leaves (dots). We work with $T$ and not with $S$ because the leaves of $S$ do not correspond to a connected component but to a void subset. We denote each vertex of $T$ as $v_{T}$. Remark that the vertex $v_{T}$ at the layer $l$ corresponds to a set of connected cubes in $\mathcal{D}_{j}$, with $j=M_{Y}-l$. For $V L$ fixed, we know the number of cubes at this scale belonging to $v_{T}$, hence also the number of vertices $v \in V_{d}$ localized in some cube of $v_{T}$. We denote this number by $n\left(v_{T}\right)$. This satisfies $n\left(v_{T}\right)=\sum_{\Delta \in v_{T}} n_{d}(\Delta)$.

Now we visualize the sums using a set of arrows on the rooted tree $T$. For each link of type $v$ (which corresponds to a vertical link $l \in v L$ ) between a vertex $v_{T}^{\prime}$ and its ancestor in the tree $v_{T}$, we draw an arrow starting at $v_{T}$ and going up, and stop the arrow at the vertex $v_{T}^{\prime \prime}$ corresponding to the connected subpolymer at scale $i_{v_{l}}$ containing the cube $\Delta_{v_{l}}$, where $v_{l}$ is the vertex associated to the link (see Fig.13).

Therefore $n\left(v_{T}\right)$ actually corresponds to the number of arrows which end at vertex $v_{T}$ in the tree $S$. Let $d\left(v_{T}\right)$ be the number of arrows departing from $v_{T}$. For any line $l$ of $T$ let us call $t(l)$ the traffic over $l$, namely the number of arrows flying above line $l$. We have obviously at any vertex $v_{T}$ of the tree a conservation law. If $l_{0}\left(v_{T}\right)$ is the trunk arriving at node $v_{T}$ from below in the tree, and $l_{1}\left(v_{T}\right), \ldots l_{p}\left(v_{T}\right)$ the branches going up from $v_{T}$, we have $t\left(l_{0}\right)+d\left(v_{T}\right)=n\left(v_{T}\right)+\sum_{i=1}^{p} t\left(l_{i}\left(v_{T}\right)\right)$.

Now the integration of the $w^{\prime}$ factors gives exactly $\prod_{l \in L(T)} 1 / t(l)$, where $L(T)$ is the set of lines in $T$ of type $v$. This can be seen as each such line corresponds to a vertical link $l \in v L$ that is to the introduction of a specific $w_{l}^{\prime}$ parameter (it is not every line of $T$, because there can be $f$ links too, 


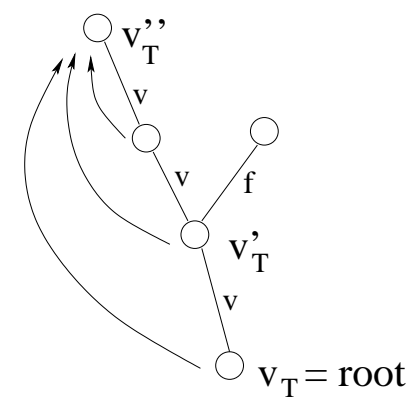

Figure 13: Example of arrow system

see Fig.13). Indeed the power of that $w^{\prime}$ factor to integrate is then exactly $t(l)-1$ for that line $l$. The -1 is there because the derivation with respect to $w^{\prime}$ erased the factor $w^{\prime}$ for the $v$ link created, but it did not erase all other $w^{\prime}$ factors for the other $v$ links going up through that line.

We can now decide to fix the numbers $n\left(v_{T}\right)$ and $d\left(v_{T}\right)$ of arrows arriving and departing at $v_{T}$. (IV.235) is then written as

$$
\left[\prod_{v_{T}} \sum_{n\left(v_{T}\right)} \sum_{d\left(v_{T}\right)}\right] \sum_{s y s t} \prod_{l \in L(T)} 1 / t(l)\left[\prod_{v_{T}} \sum_{\left\{n_{d}(\Delta)\right\}}\left(\prod_{v \in v_{T}} \sum_{\Delta_{v} \in v_{T}}\right)\right] 1
$$

$\sum_{\text {syst }}$ is the sum over all systems of arrows compatible with $n\left(v_{T}\right)$ and $d\left(v_{T}\right)$, $\left\{n_{d}(\Delta)\right\}$ chooses the number of vertices localized in each $\Delta \in v_{T}$ with the condition $\sum_{\Delta \in v_{T}} n_{d}(\Delta)=n\left(v_{T}\right)$ and $\prod_{v \in v_{T}} \sum_{\Delta_{v} \in v_{T}}$ chooses for each vertex localized in $v_{T}$ by the arrow system, the localization cube $\Delta_{v}$. The sums over $n\left(v_{T}\right)$ and $d\left(v_{T}\right)$ will be performed later and will cost at most $C^{|Y|}$.

Let us perform first the sum over $\left\{n_{d}(\Delta)\right\}$ and $\Delta_{v}$.

$$
\prod_{v \in v_{T}} \sum_{\Delta v \in v_{T}} 1 \leq \frac{n\left(v_{T}\right) !}{\prod_{\Delta \in v_{T}} n_{d}(\Delta) !} \text { and } \sum_{\left\{n_{d}(\Delta)\right\}_{\Delta \in v_{T}}} \leq 2^{\left|v_{T}\right|+n\left(v_{T}\right)}
$$

where $\left|v_{T}\right|$ is the number of cubes in $v_{T}$ and we applied $\sum_{\left\{n_{d}(\Delta)\right\}_{\Delta \in v_{T}}} 1 \leq$ $2^{\left|v_{T}\right|+n\left(v_{Y}\right)}$ (by a well known combinatoric trick, $\sum_{i_{1}, i_{2}, \ldots i_{p} \mid \sum i_{j}=m} 1 \leq 2^{m+p-1} \leq$ $\left.2^{2 m-1}\right)$. Therefore

$$
\left[\prod_{v_{T}} \sum_{\left\{n_{d}(\Delta)\right\}_{\Delta \in v_{T}}}\left(\prod_{v \in v_{T}} \sum_{\Delta_{v} \in v_{T}}\right)\right] 1 \leq C^{|Y|} \frac{\prod_{v_{T}} n\left(v_{T}\right) !}{\prod_{\Delta} n_{d}(\Delta) !}
$$


Indeed the number of vertical links of type $v$ is at most $\left|V_{T}\right|-1$ where $\left|V_{T}\right|$ is the number of vertices in $T$. Therefore we have $\sum_{v_{T}} n\left(v_{T}\right)=m \leq\left|V_{T}\right|-1 \leq$ $|Y|$.

Now we perform the sum over the arrow system. Remark that once the numbers $n\left(v_{T}\right)$ and $d\left(v_{T}\right)$ are fixed, the traffic numbers $t(l)$ are also known, since for any line the traffic $t(l)$ is equal to the sum of all arrows arriving in the subtree for which $l$ is the trunk, minus the number of arrows departing in that subtree (because arrows always go upwards in the tree, so the ones departing in the subtree have to end there too).

Now, it is easy to check that the complete choice over the system of arrows consists, for each node $v_{T}$ of the tree, in choosing by multinomial coefficients the $n\left(v_{T}\right)$ ones from the arriving traffic $t\left(l_{0}\right)$ which stop at $v_{T}$, and then which of the remaining ones go into which subbranches. This costs exactly a factor

$$
A\left(v_{T}\right)=\frac{t\left(l_{0}\left(v_{T}\right)\right) !}{n\left(v_{T}\right) ! \prod_{i} t^{\prime}\left(l_{i}\left(v_{T}\right)\right) !}
$$

where $t^{\prime}\left(l_{i}\right)=t\left(l_{i}\right)-1$ if $l_{i} \in L(T)$, that is if there is one departing arrow from node $v_{T}$ flying over line $l_{i}$ corresponding to a vertical link of type $v$ attaching the vertex $v_{T}^{\prime}$ at the upper end of line $l_{i}$ to its ancestor $v_{T}$; and $t^{\prime}\left(l_{i}\right)=t\left(l_{i}\right)$ otherwise (actually in that last case, $t^{\prime}\left(l_{i}\right)=t\left(l_{i}\right)=0$ because that link must be of type $f$ therefore no vertex from a higher scale can be associated to a vertical link at a lower scale). Therefore we have to bound

$$
\left[\prod_{v_{T}} \frac{t\left(l_{0}\left(v_{T}\right)\right) !}{n\left(v_{T}\right) ! \prod_{i} t^{\prime}\left(l_{i}\left(v_{T}\right)\right) !}\right]\left[\prod_{l \in L(T)} 1 / t(l)\right]=\left[\prod_{v_{T}} \frac{1}{n\left(v_{T}\right) !}\right][A . B]
$$

where $A$ is $\prod_{v_{T}} A\left(v_{T}\right)=\prod_{v_{T}} \frac{t\left(l_{0}\left(v_{T}\right)\right) !}{\prod_{i} t^{\prime}\left(l_{i}\left(v_{T}\right)\right) !}$ and where $B$ is our good factor coming from the $w^{\prime}$ integrals, namely $\prod_{l \in L(T)} 1 / t(l)$.

Lemma IV.7.1b For any tree and any choice of the numbers $n\left(v_{T}\right)$ and $d\left(v_{T}\right)$ (which determine the traffic numbers $t(l)$, as said above), we have A.B $=1$ (exactly!)

Proof By induction, starting from the leaves of the tree towards the trunk, we see that this is true.

For instance from a leaf $v_{T}$ of a tree, we have an apparently bad factor $t\left(l_{0}\left(v_{T}\right)\right)$ ! in $A$, where because we are at a leaf, $t\left(l_{0}\left(v_{T}\right)\right)=n\left(v_{T}\right)$ (all arrows 
must end at $v_{T}$, because there is nothing beyond if $v_{T}$ is a leaf). But then if at the node $v_{T}^{\prime}$ below that line $l_{0}\left(v_{T}\right)$ there is a departing arrow flying over $l_{0}\left(v_{T}\right)$, we have a factor $1 / t\left(l_{0}\left(v_{T}\right)\right)$ from $B$, and $l_{0}\left(v_{T}\right)=l_{i}\left(v_{T}^{\prime}\right)$ for some $i$. Combining the factor $1 / t\left(l_{0}\left(v_{T}\right)\right)$ from $B$ and the factorial $t^{\prime}\left(l_{i}\left(v_{T}^{\prime}\right)\right) !=$ $\left[t\left(l_{0}\left(v_{T}\right)\right)-1\right]$ ! in $A$ at the next node, we can reconstruct a denominator $1 / t\left(l_{0}\left(v_{T}\right)\right)$ !, which exactly cancels our bad factor $t\left(l_{0}\left(v_{T}\right)\right)$ !. Doing that for all leaves above $v_{T}^{\prime}$, we erase all bad factors and remain with exactly the numerator of the $A$ factor at node $v_{T}^{\prime}$, namely $\left[t\left(l_{0}\left(v_{T}^{\prime}\right)\right)\right]$ !. Continuing this way towards the bottom of the tree, we are finally left with a single factorial of the traffic, namely $\left[t\left(l_{0}\left(v_{T 0}\right)\right)\right]$ ! which is the last traffic at the trunk. But this traffic is 1 ! Therefore $A \cdot B=1$. This ends the proof of Lemma IV.7.1b四.

Now, the factor $\prod_{v_{T}} \frac{1}{n\left(v_{T}\right) !}$ cancels the corresponding factor on the numerator in (IV.238), while the $\prod_{\Delta} \frac{1}{n_{d}(\Delta) !}$ is kept outside. Finally we check that

$$
\left[\prod_{v_{T}} \sum_{n\left(v_{T}\right)} \sum_{d\left(v_{T}\right)}\right] 1 \leq C^{|Y|} .
$$

As for (IV.237-[V.238) we have $\sum_{v_{T}} n\left(v_{T}\right)=\sum_{v_{T}} d\left(v_{T}\right)=m \leq\left|V_{T}\right|-1 \leq|Y|$ and we apply $\sum_{i_{1}, i_{2}, \ldots i_{p} \mid \sum i_{j}=m} 1 \leq 2^{m+p-1} \leq 2^{2 m-1}$. This ends the proof of Lemma IV.7.1a.

\section{IV.7.2 Extracting a global factor $|\lambda|$}

The last sum over $M_{Y}$ will cost an extra logarithm. Therefore, in order to prove $\sum_{0 \in Y}^{Y}\left|A_{c}(Y)\right| L^{|Y|} \leq 1$ we must ensure that we can extract at least one factor $|\lambda|$ from the sum\& This is not trivial because we have only a fraction $|\lambda|^{\frac{1}{16}}$ per vertex $v \in V_{d}$. If $\left|V_{d}\right| \geq 17$ we can extract the factor $|\lambda|$ to sum over $M_{Y}$ and keep a remaining small factor $|\lambda|^{\left|V_{d}\right| /(16 \times 17)}=|\lambda|^{\left|V_{d}\right| / 272}$ per vertex. The case $\left|V_{d}\right| \leq 16$ is more delicate. Remark that, when $\left|V_{d}\right| \leq 16$, the Hadamard bound is simpler in the sense that we do not need to pay any logarithm (see case 2 in IV.3.2) or any factor $n_{\Delta}, n(\Delta)$ (see case 1 and 5 in

\footnotetext{
${ }^{10}$ This lemma is a particular variation on well known combinatoric identities $[\mathrm{BF}][\mathrm{DR} 2$, Appendix B1].

${ }^{11}$ In fact to perform a Mayer expansion, we need only to control $\sum_{0 \in Y}^{Y}$ with $M_{Y}$ fixed in our main result (III.103). However we prove the slightly stronger result (III.105) for simplicity, since it is also true.
} 
IV.3.2) to choose the contractions as the number of choices to contract a field with an antifield are bounded by $2 \cdot 16$. The only logarithms appearing are then the ones given by the sums over possible attributions ( the $\sum_{j}$ in the Hadamard bound).

We distinguish two situations:

- $\left|V_{d}\right| \leq 16$ and $|Y|=\left|V_{d}\right|$. In this case we have at most 17 energy scales, therefore any sum over scale attributions costs just a factor 17, hence the Hadamard bound does not produce any logarithm. This means that the three fields (antifields) of type $\alpha_{h} \neq 5$ hooked to the vertex $v \in V_{d}$ still have their factor $|\lambda|^{\frac{1}{4}}$, therefore we have a factor $|\lambda|^{\frac{3\left|V_{d}\right|}{4}}|\lambda|^{\frac{\left|V_{d}\right|}{16}}=|\lambda|^{\frac{13\left|V_{d}\right|}{16}}$. Now, for $\left|V_{d}\right|>1$ we can extract a factor $|\lambda|$. Otherwise, if $\left|V_{d}\right|=1$, we have a polymer reduced to one or two cubes, therefore there is no logarithms. We can extract the complete coupling constant for the unique vertex. Remark that in this case we have not extracted a small factor $g$ for the cube, but only a factor $K$. Nevertheless this is only one term of the sum (only the polymers with $|Y|=1)$.

- $\left|V_{d}\right| \leq 16$ and $|Y|>\left|V_{d}\right|$. In this case we must have at least $|Y|-\left|V_{d}\right|$ vertical links of type $f$, therefore there must be at least 2 vertices with some derived fields hooked: $\left|V_{d}^{\prime}\right| \geq 2$. Let us say that the lowest $f$-link is at scale $j$. At lower scale there can be only $v$-links, therefore there are at most 16 scales. As $M_{Y}-j \leq 16$ the set of attributions for six fields derived to give the $f$-link has at most size $M_{Y}-j \leq 16$, therefore these links do no give any logarithm, and we have a factor $|\lambda|^{6 / 4}<|\lambda|$.

\section{IV.7.3 Remaining sums}

Now the remaining sum is

$$
\begin{aligned}
& \sum_{\substack{Y \\
0 \in Y}}\left|A_{c}(Y)\right| L^{|Y|} \leq|\lambda| \sum_{M_{Y}} \sum_{S} \sum_{V L} \sum_{B_{S}}(g L C)^{|Y|} \\
& \sum_{n \geq 1}\left(C M^{13}\right)^{n} \frac{1}{n !}|\lambda|^{\frac{\left|V_{d} \cup V_{b}\right|}{272}} K^{\left|\bar{V}_{d} \backslash V_{b}\right|} \sum_{V_{d}, \alpha_{V_{d}}} \sum_{a, b, R} \sum_{\left\{v_{l}\right\}_{l \in v L}} \\
& \sum_{n_{V_{d}} \sigma_{V_{d}} \rho_{V_{d}}} \sum_{\left\{n_{\Delta}\right\}_{\Delta \in B_{S}}} \sum_{\Delta_{V_{d}}^{c}}\left[\prod_{\Delta \in B_{S}} n_{\Delta} !\right]\left[\prod_{j=m_{Y}}^{M_{Y}-1} \prod_{k=1}^{c_{j}} M^{-5 \varepsilon^{\prime}}\right]
\end{aligned}
$$


where all constants have been inserted in $C$ and the factor $\left[\prod_{\Delta \in Y} n_{d}(\Delta) !\right]$ coming from ([V.230) is compensated by $\left[\prod_{\Delta \in Y} \frac{1}{n_{d}(\Delta) !}\right]$ coming from Lemma IV.7.1a.

Sum over $\left\{n_{\Delta}\right\}$ and $\Delta_{V}^{c}$. These sums are bounded as follows.

$$
\sum_{\left\{n_{\Delta}\right\}_{\Delta \in B_{S}}} \sum_{\Delta_{\bar{V}_{d}}^{c}} \prod_{\Delta \in B_{S}}\left[n_{\Delta} !\right] \leq \sum_{\left\{n_{\Delta}\right\}_{\Delta \in B_{S}}} \frac{\left|\bar{V}_{d}\right| !}{\prod_{\Delta \in B_{S}} n_{\Delta} !} \prod_{\Delta \in B_{S}} n_{\Delta} ! \leq\left|\bar{V}_{d}\right| ! 2^{|Y|+n}
$$

where we applied

$$
\sum_{\left\{n_{\Delta}\right\} \Delta \in B_{S}} 1 \leq 2^{|Y|+n}
$$

as $\sum_{\Delta \in B_{S}} n_{\Delta}=\left|\bar{V}_{d}\right| \leq n$

Sum over $\left\{v_{l}\right\}_{l \in v L}$ This sum actually consumes a fraction of the global factorial, namely

$$
\frac{1}{n !} \sum_{\left\{v_{l}\right\}_{l \in v L}} 1 \leq \frac{1}{n !}\left[n(n-1)(n-2) \ldots\left(n-\left|V_{d}\right|+1\right)\right]=\frac{1}{\left|\bar{V}_{d}\right| !}
$$

where we applied $n-\left|V_{d}\right|=\left|\bar{V}_{d}\right|$.

Sum over $\sigma_{v_{d}}, n_{V_{d}}, a, b, R, V_{d}, \rho_{V_{d}}$ and $\alpha_{V_{d}}$. The sum over $\sigma_{v_{d}}$ costs at most a factor 4 ! per vertex, the sum over $n_{V_{d}}$ at most a factor 4 per vertex, the sums over $a, b$ and $R$ a factor 2 per field, the sum over $V_{d}$ a factor 2 per vertex, the sum over $\rho_{V_{d}}$ a factor 2 per field and finally the sum over $\alpha_{V_{d}}$ a factor 4 per vertex. Therefore

$$
\sum_{V_{d}, \alpha_{V_{d}}} \sum_{a, b, R} \sum_{n_{V_{d}} \sigma_{V_{d}}} \leq C^{n}
$$

The remaining bound is now

$$
\begin{aligned}
& \sum_{\substack{Y \\
0 \in Y}}\left|A_{c}(Y)\right| L^{|Y|} \leq|\lambda| \sum_{M_{Y}} \sum_{S} \sum_{V L} \sum_{B_{S}}(g L C)^{|Y|} \\
& \sum_{n \geq 1}\left(C M^{13}\right)^{n}|\lambda|^{\frac{\left|V_{d} \cup V_{b}\right|}{272}} K^{\left|\bar{V}_{d} \backslash V_{b}\right|}\left[\prod_{j=m_{Y}}^{M_{Y}-1} \prod_{k=1}^{c_{j}} M^{-5\left(1-2 \varepsilon^{\prime}\right)}\right]
\end{aligned}
$$


where all constants have been inserted in $C$ and the factorial $\left|\bar{V}_{d}\right|$ ! in (IV.243) has been canceled by the factor $\frac{1}{\left|V_{d}\right| !}$ in (IV.245). Now

$$
\begin{aligned}
& \sum_{n \geq 1}\left(C M^{13}\right)^{n}|\lambda|^{\frac{\left|V_{d} \cup V_{b}\right|}{272}} K^{\left|\bar{V}_{d} \backslash V_{b}\right|}= \\
& \sum_{\left|V_{d} \cup V_{b}\right| \geq 1}\left(C M^{13}|\lambda|^{1 / 272}\right)^{\left|V_{d} \cup V_{b}\right|} \sum_{\left|\bar{V}_{d} \backslash V_{b}\right| \geq 0}\left(C M^{13} K\right)^{\left|\bar{V}_{d} \backslash V_{b}\right|} \leq C
\end{aligned}
$$

for $\lambda$ and $K$ small enough, depending on $M$. The choice of $B_{S}$ costs a factor 2 per cube so finally we have to bound

$$
|\lambda| \sum_{M_{Y}} \sum_{S} \sum_{V L}(g L C)^{|Y|}\left[\prod_{j=m_{Y}}^{M_{Y}-1} \prod_{k=1}^{c_{j}} M^{-5 \varepsilon^{\prime}}\right]
$$

Sum over $S$ and $V L \quad$ These sums are performed together. For this purpose we reorganize the sum as follows:

$$
\begin{aligned}
& \sum_{S} \sum_{V L}(g L C)^{|Y|}\left[\prod_{j=m_{Y}}^{M_{Y}-1} \prod_{k=1}^{c_{j}} M^{-5 \varepsilon^{\prime}}\right] \leq \sum_{p \geq 1}(8 g L C)^{p^{0}} \\
& \sum_{d^{0} \geq 0} \prod_{i=1}^{d^{0}}\left[\sum_{p_{i}^{1} \geq 1}(8 g L C)^{p_{i}^{1}} M^{-5 \varepsilon^{\prime}} \sum_{d_{i}^{1} \geq 0} \prod_{i^{\prime}=1}^{d_{i}^{1}}\left[\sum_{p_{i^{\prime}} \geq 1}(8 g L C)^{p_{i^{\prime}}^{2}} M^{-5 \varepsilon^{\prime}} \sum_{d_{i^{\prime}} \geq 0} \ldots\right]\right]
\end{aligned}
$$

where $p^{0}$ is the number of cubes in the connected subpolymer at the layer $l=$ 0 (corresponding to the scale $M_{Y}$ ), $d^{0}$ the number of connected components at the scale $M_{Y}-1$ (circles in the rooted tree) connected to the root, $p_{i}^{1}$ the number of cubes for the connected subpolymer $i$ and so on. The factor 8 include a factor 2 to decide, for each vertical link, whether it is a $v$ or $f$ link, a factor 2 to decide for any cube of the connected subpolymer if it is going to a give a dot or not in $S$ at the next layer (see Fig. 7), and finally a factor $2^{p}$ to decide the remaining positive numbers $V L$ for the circle links of $S$ (since they are strictly positive and their sum is $p$ ).

The products stop at $p^{M_{Y}}$ as this is the maximal number of layers. We remark that for the root we do not have any vertical link, hence no vertical decay $M^{-5 \varepsilon^{\prime}}$. 
We start computing this formula from leaves, which correspond to $d=0$. Assuming $g L C \leq 1 / 16$ and $M^{-5 \varepsilon^{\prime} / 2} \leq 1 / 2$ we have

$$
\sum_{p \geq 1}(8 g L C)^{p} M^{-5 \varepsilon^{\prime}} \leq \frac{1}{2} M^{-5 \varepsilon^{\prime} / 2}
$$

Now we can perform the sum over $d$ at the previous layer

$$
\sum_{d \geq 0}\left(M^{-5 \varepsilon^{\prime} / 2}\right)^{d} \leq 2
$$

and at each layer we compensate the factor 2 by the new factor $M^{-5 \varepsilon^{\prime} / 2} \leq$ $1 / 2$.

Therefore we can sum over all layers until the root, and the result is bounded by 2 because the last layer has no $M^{-5 \varepsilon^{\prime}}$ factor.

Sum over $M_{Y}$ This sum is finally bounded as announced by our spared factor $\lambda$

$$
\sum_{\substack{Y \\ 0 \in Y}}\left|A_{c}(Y)\right| L^{|Y|} \leq|\lambda| \sum_{M_{Y}} 2 \leq 2|\ln T||\lambda| \leq 2 K \leq 1 .
$$

for $|\lambda \ln T| \leq K$.

This ends the proof of the theorem. To summarize our conditions, for a given $L$ we compute first the constant $C$, we choose $M$ large enough (and $\lambda$ small enough) so that $g L C \leq 1 / 16$ and $M^{-5 \varepsilon^{\prime} / 2} \leq 1 / 2$, and we restrict again $\lambda$ so that $C M^{13} \lambda^{1 / 272} \leq 1 / 2$. These restrictions on $\lambda$ are therefore enforced solely by taking $K$ small enough depending on $L$, which is our theorem. 


\section{Appendix A}

In section II. 4 we have introduced band decoupling on the position space, and defined, for each band $j$ the characteristic function $\Omega_{j}$. Let us introduce the following generalization of ([I.31):

$$
\begin{aligned}
\Omega_{j} & =\left\{(\vec{x}, t) \mid M^{j-1} \leq(1+|\vec{x}|)^{\frac{1}{2}+\alpha}(1+f(t)+|\vec{x}|)^{\frac{1}{2}-\alpha}<M^{j}\right. & \} j \leq j_{M} \\
& =\left\{(\vec{x}, t) \mid M^{j_{M}} \leq(1+|\vec{x}|)^{\frac{1}{2}+\alpha}(1+f(t)+|\vec{x}|)^{\frac{1}{2}-\alpha}\right. & \} j=j_{M}
\end{aligned}
$$

To select the optimal value for $\alpha$ we must insert auxiliary scales as in section II.4 and estimate the scaled decay of the propagator $C^{j k}$, as a function of $\alpha$. We insert auxiliary scale decomposition as in (11.36).

Spatial constraints The constraints on spatial positions now are:

- if $j<j_{M}$ and $k>0$ there is a non zero contribution only for

$$
M^{j} M^{-k\left(\frac{1-2 \alpha}{1+2 \alpha}\right)} M^{-\frac{2}{1+2 \alpha}} 2^{-\frac{1-2 \alpha}{1+2 \alpha}} \leq(1+|\vec{x}|) \leq M^{j} M^{-k\left(\frac{1-2 \alpha}{1+2 \alpha}\right)} M^{\frac{1-2 \alpha}{1+2 \alpha}}
$$

- for $j \leq j_{M}$ and $k=0$ there is a non zero contribution only for

$$
M^{j} M^{-\frac{2}{1+2 \alpha}} 2^{-\frac{1-2 \alpha}{1+2 \alpha}} \leq(1+|\vec{x}|) \leq M^{j}
$$

- for $j=j_{M}+1$ there is a non zero contribution only for

$$
M^{j_{M}} 2^{-\frac{1-2 \alpha}{1+2 \alpha}} \leq(1+|\vec{x}|)
$$

Scaled decay of the propagator Now for each $j$ and $k$ we can estimate the scaled decay of the propagator $C^{j, k}$. We distinguish three cases:

- for $j<j_{M}$ and $k>0$ we have

$$
\left|C^{j, k}(\vec{x}, t)\right| \leq M^{-2 j} M^{-k\left(\frac{4 \alpha}{1+2 \alpha}\right)} M^{\frac{3+2 \alpha}{1+2 \alpha}} 2^{\frac{1-2 \alpha}{1+2 \alpha}} \chi_{j, k}(\vec{x}, f(t))
$$

where the function $\chi_{j, k}$ is defined by

$$
\begin{array}{rlll}
\chi_{j, k}(\vec{x}, t) & =1 & & \text { if }|\vec{x}| \leq M^{j} M^{-k\left(\frac{1-2 \alpha}{1+2 \alpha}\right)} M^{\frac{1-2 \alpha}{1+2 \alpha}}, f(t) \leq M^{j+k} \\
& =0 & & \text { otherwise }
\end{array}
$$


- for $j \leq j_{M}$ and $k=0$ we have

$$
\left|C^{j, 0}(\vec{x}, t)\right| \leq M^{-2 j} M^{\frac{4}{1+2 \alpha}} 2^{2\left(\frac{1-2 \alpha}{1+2 \alpha}\right)} \chi_{j, 0}(\vec{x}, f(t))
$$

where the function $\chi_{j, 0}$ is defined by

$$
\begin{aligned}
& \chi_{j, 0}(\vec{x}, t)=1 \quad \text { if }|\vec{x}| \leq M^{j}, f(t) \leq M^{j} \\
& =0 \text { otherwise }
\end{aligned}
$$

- for $j=j_{M}+1$ we have

$$
\left|C^{j_{M}+10}(\vec{x}, t)\right| \leq M^{-2 j_{M}} 2^{2\left(\frac{1-2 \alpha}{1+2 \alpha}\right)} \chi_{j_{M}+1,0}(f(t)) \frac{K_{p}}{\left(1+M^{-j_{M}}|\vec{x}|\right)^{p}}
$$

where the function $\chi_{j_{M}+1,0}$ is defined by

$$
\begin{aligned}
& \chi_{j_{M}+1,0}(t)=1 \text { if } f(t) \leq M^{j_{M}} \\
& =0 \quad \text { otherwise }
\end{aligned}
$$

and the spatial decay for $|\vec{x}|$ comes from the decay of the function $F$ in (II.7).

Integration volume The region of spatial integration (for a scale propagator) is now fixed by the $\chi_{j, k}$ domain. Therefore

- for $j<j_{M}$ and $k>0$ we have

$$
V_{j, k}=|\vec{x}|^{3} f(t) \leq M^{4 j} M^{-k\left(\frac{2-8 \alpha}{1+2 \alpha}\right)} M^{3\left(\frac{1-2 \alpha}{1+2 \alpha}\right)}
$$

- for $j \leq j_{M}$ and $k=0$ we have

$$
V_{j, k}=|\vec{x}|^{3} f(t) \leq M^{4 j}
$$

- for $j=j_{M}+1$ we have

$$
V_{j, k}=|\vec{x}|^{3} f(t) \leq M^{4 j_{M}}
$$


As we have seen, the tree propagator is used in two cases, namely to bound the sum over cubes in the Hadamard bound (see ([V.140) ) and to perform the sum over trees. In the Hadamard bound we must have

$$
F_{j k}=:\left|C^{j k}\right|^{2} M^{4 j} M^{k} \leq K M^{-\varepsilon k}
$$

for some constants $K, \varepsilon>0$ ( $K$ is actually proportional to some constant power of $M$ ). The decay $M^{-\varepsilon k}$ is necessary to sum over $k$. Inserting the $\alpha$ depending bounds for $C^{j k}$ we have, for $k>0$

$F_{j k} \leq M^{-4 j} M^{-k\left(\frac{8 \alpha}{1+2 \alpha}\right)} M^{2 \frac{3+2 \alpha}{1+2 \alpha}} 2^{2 \frac{1-2 \alpha}{1+2 \alpha}} M^{4 j} M^{k}=M^{k\left[1-\left(\frac{8 \alpha}{1+2 \alpha}\right)\right]} M^{2 \frac{3+2 \alpha}{1+2 \alpha}} 2^{2 \frac{1-2 \alpha}{1+2 \alpha}}$

and (A.14) is true for

$$
1-\left(\frac{8 \alpha}{1+2 \alpha}\right)<0 \Rightarrow \alpha>\frac{1}{6}
$$

On the other hand when summing over the tree structure we must ensure that

$$
F_{j k}=:\left|C^{j k}\right| V_{j k} \leq K M^{2 j} M^{-\varepsilon k}
$$

for some constants $K, \varepsilon>0$ ( $K$ is actually proportional to some constant power of $M$ ). Again the decay $M^{-\varepsilon k}$ is necessary to sum over $k$. Inserting the values for $\left|C^{j k}\right|$ and $V_{j k}$ we have

$$
\begin{aligned}
F_{j k} & \leq M^{-2 j} M^{-k\left(\frac{4 \alpha}{1+2 \alpha}\right)} M^{\frac{3+2 \alpha}{1+2 \alpha}} 2^{\frac{1-2 \alpha}{1+2 \alpha}} M^{4 j} M^{-k\left(\frac{2-8 \alpha}{1+2 \alpha}\right)} M^{3\left(\frac{1-2 \alpha}{1+2 \alpha}\right)} \\
& \leq M^{2 j} M^{-k\left(\frac{2-4 \alpha}{1+2 \alpha}\right)} M^{2\left(\frac{3-2 \alpha}{1+2 \alpha}\right)} 2^{\frac{1-2 \alpha}{1+2 \alpha}}
\end{aligned}
$$

and (A.17) is true for

$$
2-8 \alpha>0 \Rightarrow \alpha<\frac{1}{2}
$$

Therefore the parameter $\alpha$ then can take values only in the open interval $\left(\frac{1}{6}, \frac{1}{2}\right)$. Actually we choose the value $\alpha=\frac{1}{4}$ which corresponds to

$$
V_{j k}=M^{4 j}
$$

For this value the band volume does not depend on $k$ which is consistent with the choice of $j$ as the real band slicing, while $k$ is just an auxiliary band slicing. 
Acknowledgment M. Disertori acknowledges partial support of NSF grant DMS 97-29992 for this work.

\section{References}

[AR1] A. Abdesselam and V. Rivasseau, Rev. Math. Phys. Vol. 9 No 2, 123 (1997)

[AR2] A. Abdesselam and V. Rivasseau, Trees, forests and jungles: a botanical garden for cluster expansions, in Constructive Physics, ed by V. Rivasseau, Lecture Notes in Physics 446, Springer Verlag, 1995.

[BF] G. A. Battle and P Federbush, A note on cluster expansions, tree graph identities, extra 1/N! factors!!! Lett. Math. Phys. 8, 55, (1984).

[BG] G. Benfatto and G. Gallavotti, Perturbation theory of the Fermi surface in a quantum liquid. A general quasi particle formalism and one dimensional systems, Journ. Stat. Phys. 59541 (1990).

[BGPS] G. Benfatto, G. Gallavotti, A. Procacci and B. Scoppola, Commun. Math. Phys. 160, 93 (1994).

[BM] F. Bonetto and V. Mastropietro, Commun. Math. Phys. 172, 57 (1995).

[DR1] M. Disertori and V. Rivasseau, Interacting Fermi liquid in two dimensions at finite temperature, Part I: Convergent Attributions.

[DR2] M. Disertori and V. Rivasseau, Interacting Fermi liquid in two dimensions at finite temperature, Part II: Renormalization,

[FMRT] J. Feldman, J. Magnen, V. Rivasseau and E. Trubowitz, An infinite Volume Expansion for Many Fermion Green's Functions, Helv. Phys. Acta 65 (1992) 679.

[FT1] J. Feldman and E. Trubowitz, Perturbation theory for Many Fermion Systems, Helv. Phys. Acta 63 (1991) 156.

[FT2] J. Feldman and E. Trubowitz, The flow of an Electron-Phonon System to the Superconducting State, Helv. Phys. Acta 64 (1991) 213.

[GN] G. Gallavotti and F. Nicolò, Renormalization theory in four dimensional scalar fields, I and II, Commun. Math. Phys. 100, 545 and 101, 247 (1985). [MR] J. Magnen and V. Rivasseau, A Single Scale Infinite Volume Expansion for Three Dimensional Many Fermion Green's Functions, Math. Phys. Electronic Journal, Volume 1, 1995.

[R] V. Rivasseau, From perturbative to constructive Renormalization, Princeton University Press, 1991. 
[S] M. Salmhofer, Continuous renormalization for Fermions and Fermi liquid theory, Commun. Math. Phys.194, 249 (1998). 